\title{
The Jovian Auroral Distributions Experiment (JADE) on the Juno Mission to Jupiter
}

\author{
D.J. McComas • N. Alexander • F. Allegrini • F. Bagenal • C. Beebe • G. Clark • \\ F. Crary - M.I. Desai - A. De Los Santos - D. Demkee · J. Dickinson · D. Everett • \\ T. Finley • A. Gribanova - R. Hill · J. Johnson - C. Kofoed • C. Loeffler · P. Louarn • \\ M. Maple • W. Mills • C. Pollock • M. Reno • B. Rodriguez • J. Rouzaud • \\ D. Santos-Costa $\cdot$ P. Valek $\cdot$ S. Weidner $\cdot$ P. Wilson $\cdot$ R.J. Wilson $\cdot$ D. White
}

Received: 1 August 2012 / Accepted: 29 April 2013 / Published online: 25 May 2013

(C) The Author(s) 2013. This article is published with open access at Springerlink.com

\begin{abstract}
The Jovian Auroral Distributions Experiment (JADE) on Juno provides the critical in situ measurements of electrons and ions needed to understand the plasma energy particles and processes that fill the Jovian magnetosphere and ultimately produce its strong aurora. JADE is an instrument suite that includes three essentially identical electron sensors (JADE-Es), a single ion sensor (JADE-I), and a highly capable Electronics Box (EBox) that resides in the Juno Radiation Vault and provides all necessary control, low and high voltages, and computing support for the four sensors. The three JADE-Es are arrayed $120^{\circ}$ apart around the Juno spacecraft to measure complete electron distributions from $\sim 0.1$ to $100 \mathrm{keV}$ and provide detailed electron pitch-angle distributions at a $1 \mathrm{~s}$ cadence, independent of spacecraft spin phase. JADE-I measures ions from $\sim 5 \mathrm{eV}$ to $\sim 50 \mathrm{keV}$ over an instanta-
\end{abstract}

D.J. McComas $(\bigotimes) \cdot$ N. Alexander · F. Allegrini · C. Beebe · G. Clark · F. Crary · M.I. Desai ·

A. De Los Santos · D. Demkee · J. Dickinson - D. Everett · T. Finley · A. Gribanova - J. Johnson ·

C. Kofoed · C. Loeffler · M. Maple · W. Mills · C. Pollock · M. Reno · B. Rodriguez · D. Santos-Costa ·

P. Valek $\cdot$ S. Weidner $\cdot$ P. Wilson $\cdot$ D. White

Southwest Research Institute, San Antonio, TX, USA

e-mail: dmccomas@swri.edu

D.J. McComas · F. Allegrini · G. Clark · M.I. Desai · P. Valek

Physics and Astronomy Department, University of Texas at San Antonio, San Antonio, TX, USA

Present address:

C. Pollock

Goddard Space Flight Center, Greenbelt, MD, USA

M. Reno

Austin Mission Consulting, Austin, TX, USA

F. Bagenal · R.J. Wilson

Laboratory for Atmospheric and Space Physics, Boulder, CO, USA

R. Hill

Baja Technologies, LLC, Tucson, AZ, USA

P. Louarn · J. Rouzaud

Institut de Recherche en Astrophysique et Planétologie (IRAP), Toulouse, France 
neous field of view of $270^{\circ} \times 90^{\circ}$ in $4 \mathrm{~s}$ and makes observations over all directions in space each $30 \mathrm{~s}$ rotation of the Juno spacecraft. JADE-I also provides ion composition measurements from 1 to 50 amu with $m / \Delta m \sim 2.5$, which is sufficient to separate the heavy and light ions, as well as $\mathrm{O}+$ vs $\mathrm{S}+$, in the Jovian magnetosphere. All four sensors were extensively tested and calibrated in specialized facilities, ensuring excellent on-orbit observations at Jupiter. This paper documents the JADE design, construction, calibration, and planned science operations, data processing, and data products. Finally, the Appendix describes the Southwest Research Institute [SwRI] electron calibration facility, which was developed and used for all JADE-E calibrations. Collectively, JADE provides remarkably broad and detailed measurements of the Jovian auroral region and magnetospheric plasmas, which will surely revolutionize our understanding of these important and complex regions.

Keywords Juno · JADE · Plasma energy particles · Jupiter · Aurora · Auroral regions · Auroral distributions

\section{Acronyms}

$\begin{array}{ll}\text { ABM } & \text { Absolute Beam Monitor } \\ \text { AFT } & \text { Allowable Flight Temperature } \\ \text { ATLO } & \text { Assembly, Test, Launch and Operations } \\ \text { CEM } & \text { Channel Electron Multiplier } \\ \text { CEU } & \text { Common Electronics Unit } \\ \text { CODMAC } & \text { Committee on Data Management and Computation } \\ \text { DEF } & \text { Differential Energy Flux } \\ \text { DFL } & \text { Deflector } \\ \text { DFL-DN } & \text { Lower Deflector } \\ \text { DFL-UP } & \text { Upper Deflector } \\ \text { DM/MDNF } & \text { Differential Number Flux } \\ \text { DPID } & \text { Data Product IDentifier } \\ \text { EDAC } & \text { Error Detection and Correction } \\ \text { Ebox } & \text { Electronics Box } \\ \text { EEPROM } & \text { Electrically Erasable Programmable Read-Only Memory } \\ \text { EMI } & \text { Electromagnetic Interference } \\ \text { EOL } & \text { End of Life } \\ \text { ESA } & \text { Electrostatic Analyzer } \\ \text { FA } & \text { Flight Acceptance } \\ \text { FAST } & \text { Fast Auroral Snapshot Explorer } \\ \text { FSW } & \text { Flight Software } \\ \text { FPGA } & \text { Field Programmable Gate Array } \\ \text { FWHM } & \text { Full-Width at Half-Maximum } \\ \text { HRS } & \text { High Rate Science } \\ \text { HVENG } & \text { High Voltage Engineering } \\ \text { HV } & \text { High Voltage } \\ \text { FGH } & \text { Flux-Gate Magnetometer } \\ \text { FOV } & \text { Field of View } \\ \text { FSW } & \text { Flight Software } \\ G & \text { Geometric factor } \\ \text { G } & \text { Gauss } \\ \text { GRAV } & \text { Gravity Science } \\ \text { HVDB } & \text { High Voltage Distribution Board } \\ & \end{array}$


HVPS High Voltage Power Supply

IESD Internal Electrostatic Discharge

IPB Instrument Processing Board

JADE Jovian Auroral Distributions Experiment

JADE-E Jovian Auroral Distributions Experiment-Electron Sensor(s)

JADE-I Jovian Auroral Distributions Experiment-Ion Sensor

JEDI Jupiter Energetic particle Detector Instrument

JOI Jupiter Orbit Insertion

JPL-DG JPL Divine-Garrett empirical model

JSIB JADE Sensor Interface Board

LRS Low Rate Science

LUT Lookup Table

LVDS Low Voltage Differential Signaling

LVENG Low Voltage Engineering

LVPS Low Voltage Power Supply

MAG Magnetometer

MCP Microchannel Plate

MET Mission Elapsed Time

MFLOPS Million Floating Point Operations per Second

MWG Magnetospheric Working Group

MWR Microwave Radiometer

NRC National Research Council

NAIF NASA's Navigation and Ancillary Information Facility

NVR Non-volatile Residue

OTM Orbit Trim Maneuver

PF Protoflight

PIM Periodic Instrument Maintenance

PPS Pulse per Second

PSD Phase Space Density

REDR Reformatted Engineering Data Record

SE Secondary Electron

SEU Single Event Upset

SOAP Satellite Orbit Analysis Program

SPICE Software Process Improvement and Capability dEtermination

SRAM Static Random Access Memory

SwRI Southwest Research Institute

SwRI-DSC SwRI Santos-Costa (SwRI-DSC) physics-based model

SYS3 System III coordinate system

TOF Time of Flight

UART Universal Asynchronous Receiver/Transmitter

UTC Coordinated Universal Time

UV Ultraviolet 


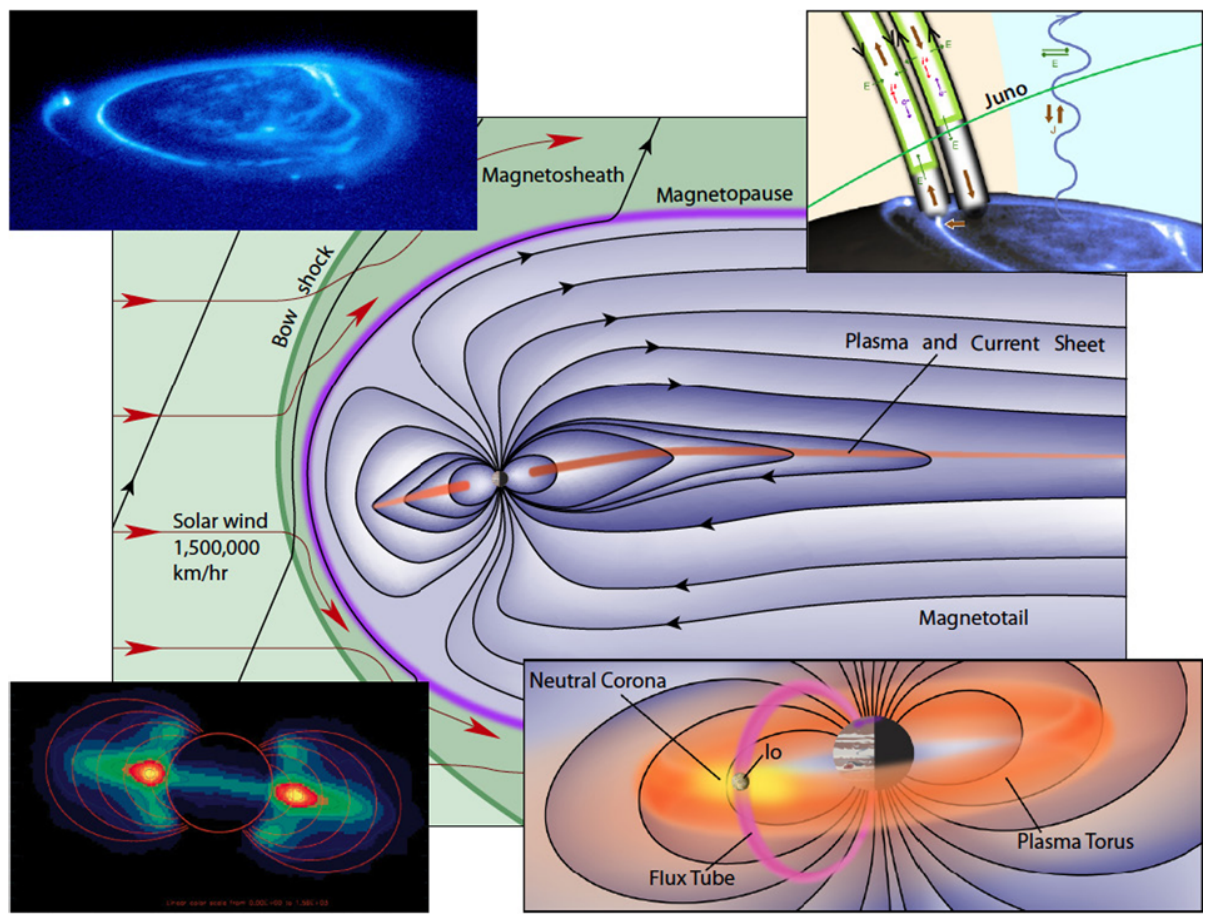

Fig. 1 The magnetosphere of Jupiter

\section{Introduction}

\subsection{Science Background and Objectives}

\subsubsection{Plasma Populations in Jupiter's Magnetosphere}

The magnetosphere of Jupiter is the largest object within the heliosphere, occupying a volume over a thousand times that of the Sun (Fig. 1). The several different plasma populations play a variety of key roles in this vast magnetosphere. Volcanic gases escaping from Io are ionized at a rate of about $1 \mathrm{ton} / \mathrm{s}$. These ionized dissociation products of $\mathrm{SO}_{2}$ are picked up by Jupiter's magnetic field, accelerated to corotation speeds $(\sim 60 \mathrm{~km} / \mathrm{s})$, and form a torus of dense $\left(\sim 2000 \mathrm{~cm}^{-3}\right)$ plasma that spreads radially outwards, into a plasma disk, over timescales of weeks. Rather than cooling (from temperatures of $\sim 100 \mathrm{eV}$ ) on expansion, the plasma is observed to heat up (to $\sim 10 \mathrm{~s}$ of $\mathrm{keV}$ energies) by an as yet unknown mechanism.

The magnetospheric plasma is coupled to Jupiter's ionosphere via electrical currents that are carried by the plasma particles, likely accelerated by electric fields directed along the local magnetic field. These accelerated particles bombard Jupiter's atmosphere and excite auroral emissions at wavelengths ranging from $\mathrm{X}$-rays to radio waves. The magnetospheric plasma is thought to leave the magnetosphere primarily down the long magnetotail that extends away from the Sun in some form to at least the orbit of Saturn. Jupiter's giant magnetosphere presents a substantial obstacle to the solar wind. The mechanisms by which the solar wind and magnetospheric plasmas interact, and the consequences of such interactions, 
remain issues of strong disagreement and debate. A full description of the Jovian magnetosphere is given in the accompanying magnetospheric science overview paper (Bagenal et al. 2013, this issue). Here we summarize past plasma measurements within the magnetosphere of Jupiter, the outstanding issues, and how JADE addresses important science objectives.

\subsubsection{Jupiter's Unexplored Polar Regions}

The primary JADE objective is to explore Jupiter's heretofore unexplored polar magnetosphere regions. (We note that Ulysses only sampled mid-latitudes and that all other Jovian measurements have been even more equatorial.) What we expect to see there is partly guided by terrestrial magnetospheric observations over the poles at Earth (e.g. from the FAST spacecraft, see reviews by Carlson et al. 1998; and chapters in Paschmann et al. 2003) and from previous observations in Jupiter's equatorial region (reviewed by Khurana et al. 2004; Krupp et al. 2004) as well as observations of Jupiter's powerful aurora (reviewed by Clarke et al. 2004). JADE measures the plasma flow velocity, density, temperature, composition, as well as the flux of electron and ion particle populations that are expected to be streaming both towards and away from the planet along the local magnetic field. Key to understanding the structure and processes of the polar region are high cadence measurements of energy and angular distributions. As Juno (Bolton et al. 2013, this issue) passes over the poles its speed is $\sim 50 \mathrm{~km} / \mathrm{s}$, and it is spinning at $2 \mathrm{rpm}$. When JADE takes data at its fastest cadence, it will resolve structures on the order of $50 \mathrm{~km}$ for electron pitch-angle distributions and $1500 \mathrm{~km}$ for complete 3-D ion distributions. Auroral observations suggest that Juno will move through structures that are $\sim 1000 \mathrm{~km}$ across (Clarke et al. 2004), so JADE is ideal for studying these regions.

Just as with FAST at Earth, it is vital to coordinate with Juno's other particles and fields instruments. The magnetometer (MAG; Connerney et al. 2013, this issue) detects field perturbations due to currents flowing between the ionosphere and magnetosphere. The particles carrying these currents are measured by JADE and the Jupiter Energetic-particle Detector Instrument (JEDI; Mauk et al. 2013, this issue), depending on their energy. At the same time, the Juno plasma wave instrument (Waves; Kurth et al. 2013, this issue) measures local plasma waves and radio emissions excited by non-thermal particle distributions, and auroral images are made by the remote sensing ultraviolet spectrometer (UVS; Gladstone et al. 2013, this issue), Infrared imager (JIRAM; Adriani et al. 2013, this issue), and visible light camera (Junocam; Hansen et al. 2013, this issue) on Juno as well as from Earth-based telescopes. Juno's Magnetospheric Working Group (MWG) coordinates the polar observing sequences to compile the different data sets for combined scientific analysis at the various relevant dimensions and timescales (see Bagenal et al. 2013, this issue).

\subsubsection{Mapping of Plasma Properties}

Juno's 11-day primary science orbits allow observations in the middle magnetosphere during the extended apojove parts of each orbit. The $\sim 10^{\circ}$ tilt of Jupiter's magnetic axis and $\sim 10$-hour rotation rate mean that Juno passes through the plasma disk many times. JADE not only maps out the spatial distribution of plasma properties (flow velocity, density, and temperature), it also uniquely resolves heavy and light ion composition from various sources (Io, Jupiter, and the solar wind). Voyager in situ plasma measurements indicated that $\mathrm{O}$ and $\mathrm{S}$ ions dominate the Jovian plasma environment (comprising up to $80 \%$ of the density), but the relative amounts of these dominant ions could only be measured via their spectroscopic signatures in ultraviolet (UV) emissions from the Io plasma torus (Thomas 
et al. 2004). While $\mathrm{O}+$ and $\mathrm{S}++$ cannot be separated, JADE will uniquely identify $\mathrm{O}+$ vs. S+. Measurements of ion and electron densities and temperatures by JADE are crucial in understanding magnetospheric dynamics and heating processes (Krupp et al. 2004; Bagenal and Delamere 2011).

\subsubsection{Solar Wind Interaction with Jupiter's Magnetosphere}

On approach to Jupiter and during the capture orbit, Juno spends several months on the dawn flank of the magnetosphere. The distance to the magnetopause and bow shock boundaries varies considerably with upstream solar wind dynamic pressure (Joy et al. 2002). Using measurements of the solar wind dynamic pressure measured by the Ulysses SWOOPS instrument (e.g., Bame et al. 1992) and the Joy et al. (2002) empirical functions, it is estimated that Juno will likely cross the bow shock and magnetopause 64 and 42 times $( \pm 9)$, respectively (Bagenal et al. 2013). Thus JADE will have ample opportunities to examine these critical plasma boundaries in detail.

The basic processes of the overall interaction of the solar wind with Jupiter's magnetosphere (see review by Krupp et al. 2004) have recently been questioned, with an Earth-like "Dungey Cycle" particularly cast into doubt (McComas and Bagenal 2007; Delamere and Bagenal 2010). Observations by JADE and the other Juno MWG instruments on the dawn flank of the magnetosphere, as well as over the poles, where the magnetic field may or may not connect to the complicated plasma and energetic particle structures observed down the Jovian magnetotail (McComas et al. 2007; McNutt et al. 2007), will provide critical opportunities to distinguish between these competing ideas.

\subsection{JADE Implementation, Mission Requirements and Performance}

\subsubsection{Flow Down of Science Requirements}

The National Research Council's 2003 Planetary Science Decadal Survey (NRC 2003) defined the key questions that the science community ranked as critical to understanding the Jupiter system. The Juno mission was formulated and proposed to answer these questions, and Juno's mission and system requirements can be traced back to how it answers these fundamental questions (Bolton et al. 2013, this issue). Bolton et al. provide the traceability that shows the connections from the Decadal Survey's science questions to the lower level engineering requirements that translated into Juno Level 3 Payload Requirements for JADE (Table 1). These Level 3 requirements detail the capabilities of the JADE instrument suite, which comprises three identical electron sensors (JADE-Es), an ion composition sensor (JADE-I), and a common electronics unit (EBox) that services all four sensors; the Level 3 requirements also document what JADE requires from the spacecraft (e.g. knowledge of the current time, the magnetic field, and the spin-phase of the spacecraft).

\subsubsection{Key Measurement Requirements and Performance}

The detailed Level 3 measurement requirements, measurement goals as designed, and measured capabilities actually achieved for the JADE-E and JADE-I sensors are provided in Table 1. These requirements flowed down into the Level 4 and Level 5 requirements documents used to design JADE. As the table shows, JADE meets all (and exceeds most) of its Level 3 Requirements (review after JADE-I cal section). 
Table 1 JADE level 3 requirements and calibration results

\begin{tabular}{|c|c|c|c|}
\hline Parameter & Requirement & Goal & Measured \\
\hline \multicolumn{4}{|l|}{ JADE-E } \\
\hline Energy range & $200 \mathrm{eV}$ to $40 \mathrm{keV}$ & $100 \mathrm{eV}$ to $100 \mathrm{keV}$ & $100 \mathrm{eV}$ to $95 \mathrm{keV}^{\mathrm{a}}$ \\
\hline$\Delta E / E$ & $<15 \%$ & $\sim 10 \%$ & $\sim 11 \%(\mathrm{cf}$. Table 11$)$ \\
\hline FOV & $360^{\circ} \times 60^{\circ}$ & $360^{\circ} \times 90^{\circ}$ & $360^{\circ} \times 70^{\circ}$ \\
\hline Pixel & $15^{\circ} \times 15^{\circ}$ & $7.5^{\circ} \times 5^{\circ}$ & $7.5^{\circ} \times 3-6^{\circ}$ \\
\hline Geometric factor per pixel & $10^{-3}-10^{-5} \mathrm{~cm}^{2} \mathrm{sreV} / \mathrm{eV}$ & $10^{-4} \mathrm{~cm}^{2} \mathrm{sreV} / \mathrm{eV}$ & $2-5 \times 10^{-5} \mathrm{~cm}^{2} \mathrm{sreV} / \mathrm{eV}$ \\
\hline Time resolution & $2 \mathrm{~s}$ & $1 \mathrm{~s}$ & $1 \mathrm{~s}$ \\
\hline \multicolumn{4}{|l|}{ JADE-I } \\
\hline Energy range & $13 \mathrm{eV}-20 \mathrm{keV}$ & $10 \mathrm{eV}-40 \mathrm{keV}$ & $5 \mathrm{eV}^{\mathrm{b}}-43.2 \mathrm{keV}$ \\
\hline$\Delta E / E$ & $10-30 \%$ & $20 \%$ & $\begin{array}{l}28 \% \text { to } 18 \% \\
21 \% \text { at } 10 \mathrm{keV}\end{array}$ \\
\hline FOV & $>180^{\circ} \times 60^{\circ}$ & $270^{\circ} \times 90^{\circ}$ & $270^{\circ} \times 90^{\circ}$ \\
\hline Pixel & $<30^{\circ} \times 30^{\circ}$ & $10^{\circ} \times 20^{\circ}$ & $8.5^{\circ} \times 30^{\circ}$ \\
\hline Mass range & $1-32 \mathrm{amu}$ & $1-50 \mathrm{amu}$ & $1->40^{\mathrm{c}}$ amu \\
\hline$M / \Delta M$ & $>2.5$ & $>3$ & 2.5 to $11^{\mathrm{d}}$ \\
\hline $\begin{array}{l}\text { Geometric factor } \\
\text { (at } 10 \mathrm{keV})\end{array}$ & $10^{-5}-10^{-3} \mathrm{~cm}^{2} \mathrm{sreV} / \mathrm{eV}$ & $10^{-4} \mathrm{~cm}^{2} \mathrm{sreV} / \mathrm{eV}$ & $4.0 \times 10^{-4} \mathrm{~cm}^{2} \mathrm{sreV} / \mathrm{eV}^{\mathrm{e}}$ \\
\hline Time & $8 \mathrm{~s}$ & $4 \mathrm{~s}$ & $4 \mathrm{~s}$ \\
\hline
\end{tabular}

\footnotetext{
${ }^{\mathrm{a}}$ By analysis $>30 \mathrm{keV}$

${ }^{\mathrm{b}}$ By analysis $<1 \mathrm{keV}$

${ }^{\mathrm{c}}$ Ar was the largest mass tested

${ }^{\mathrm{d}}$ Mass and energy dependent

${ }^{\mathrm{e}}$ Full FOV for events with valid TOF
}

\subsubsection{Environmental Requirements}

The Juno mission is challenging because of the difficult environment at Jupiter combined with the need for compatibility among a diverse complement of sensitive science instruments. The environmental requirements for the Juno mission are captured in the Juno Environmental Requirement Document (ERD). In the sections below we discuss these key issues and our approach to solving them.

1.2.3.1 Jovian Trapped Radiation Belts and Detector Background One of the most difficult aspects of exploring Jupiter is effectively meeting the challenge of Jupiter's large and intense trapped-radiation belts. Two key aspects of the Juno design are used to mitigate the radiation issue. The first is the selection of a polar orbit for the mission design, which largely avoids the harshest regions of the radiation belts until late in the mission. The second key to the design is the incorporation of a radiation vault architecture for the spacecraft design. Rather than shield electronics boxes individually, the Juno mission provides a large titanium vault in which almost all of the electronics are housed. The JADE suite comprises four sensors that are outside the vault on the perimeter of the spacecraft, in order to measure the Jovian plasma with relatively unobstructed fields of view (FOVs). The minimum amount of front-end electronics required to perform the initial signal processing are located within 
Fig. 2 Example of MWR notch EMI result for JADE

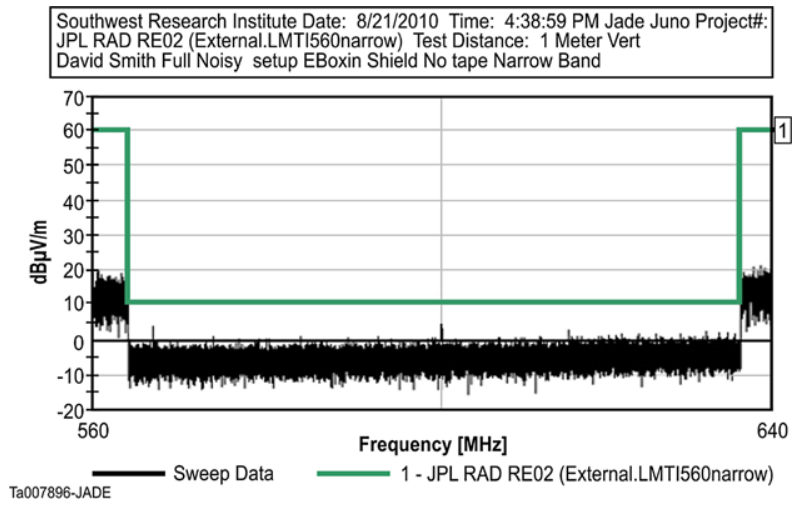

the four sensors in order to minimize shielding mass. The majority of the signal processing electronics is contained with the JADE Electronics Box (Ebox), which is located within the Juno Radiation Vault. Cables from the JADE Ebox are passed to the sensors through bulkhead connections for low-voltage signals. To increase reliability, the high-voltage cables are passed through "mouse holes" (specifically designed pass throughs incorporating EMI and radiation shielding to ensure the continuity of the radiation-limiting design) in the vault wall to minimize the number of high-voltage connections required.

While much of the work at the mission level for radiation mitigation is focused on electronics parts, science instruments such as JADE, with sensitive microchannel plate (MCP) detectors must also solve the issue of detector background caused by penetrating radiation. The JADE team used the best currently available knowledge and models of Jupiter's magnetosphere to estimate the upper and lower bounds of the signals JADE measures. These calculations and JADE's design response to the challenging penetrating particle environment is detailed in Sect. 2.5.

1.2.3.2 EMI/EMC Requirements The EMI/EMC requirements for Juno conformed to the MIL-STD-461D test, with most of the Juno-specific tailoring involving a set of deep notches for the MWR instrument's sensitive receivers in the microwave range.

The JADE-E sensors are the near field of the MWR antenna. A detailed analysis was performed by the Juno project to determine the coupling between the JADE-E sensors and the MWR antennae. Figure 2 provides an example of one of the MWR notches. In the frequency range of interest for the MWR instrument, the notch is $50 \mathrm{~dB}$ below the standard level. To mitigate the risk that JADE could corrupt the MWR observations, extensive EMI testing was done during both the engineering model (EM) and flight model (FM) development phases. During the EM EMI testing we found that special shielding and bulk-head closeout pieces were required to contain EMI and produce the notches. The JADE sensors subsequently met all MWR EMI requirements during FM qualification testing.

1.2.3.3 Magnetic Cleanliness Requirements Juno's magnetic cleanliness requirements are similar to most deep space science missions that carry magnetometers. JADE minimizes the use of magnetic or magnetizable materials. Acceptance testing at the spacecraft contractor's facility found that JADE had only very small residual magnetic moments. The JADE electronics unit had a magnitude difference of $30 \mathrm{~mA} \mathrm{~m}^{2}$ between its magnetized and demagnetized values. This equates to only a $0.01 \mathrm{nT}$ delta to the spacecraft $\mathrm{AC}$ magnetic signature, which is below the spacecraft's allocation for JADE. 
Table 2 JADE temperature limits $(\mathrm{AFT}=$ Allowable Flight Temperature, FA $=$ Flight Acceptance, $\mathrm{PF}=$ Proto-Flight)

\begin{tabular}{|c|c|c|c|c|c|c|c|c|c|c|}
\hline \multirow[t]{2}{*}{ SC element } & \multicolumn{6}{|c|}{ Operating temperatures, ${ }^{\circ} \mathrm{C}$} & \multicolumn{4}{|c|}{ Non-op temperatures, ${ }^{\circ} \mathrm{C}$} \\
\hline & $\begin{array}{l}\text { AFT } \\
\text { min }\end{array}$ & $\begin{array}{l}\text { AFT } \\
\text { max }\end{array}$ & $\begin{array}{l}\text { FA } \\
\text { min }\end{array}$ & $\begin{array}{l}\text { FA } \\
\max \end{array}$ & $\begin{array}{l}\mathrm{PF} \\
\mathrm{min}\end{array}$ & $\begin{array}{l}\mathrm{PF} \\
\max \end{array}$ & $\begin{array}{l}\text { AFT } \\
\text { min }\end{array}$ & $\begin{array}{l}\text { AFT } \\
\max \end{array}$ & $\begin{array}{l}\mathrm{PF} \\
\mathrm{min}\end{array}$ & $\begin{array}{l}\mathrm{PF} \\
\max \\
\end{array}$ \\
\hline Ion sensor & -25 & 40 & -30 & 45 & -40 & 55 & -35 & 50 & -50 & 65 \\
\hline Electron sensor & -25 & 40 & -30 & 45 & -40 & 55 & -35 & 50 & -50 & 65 \\
\hline JADE electronics & -10 & 45 & -15 & 50 & -25 & 60 & -20 & 55 & -35 & 70 \\
\hline
\end{tabular}

1.2.3.4 Spacecraft Charging The Juno spacecraft must function properly in Jupiter's strong magnetic and radiation environments. Furthermore, in order to minimize the level of spacecraft charging, all surfaces on Juno had to be ESD dissipative with a resistance $<10^{9} \mathrm{ohms}$ per square. All exposed surfaces on JADE are grounded and the MLI, radiator surfaces, and tapes used are electrically dissipative.

Additionally, Internal Electrostatic Discharge (IESD) was determined to be a significant threat owing to the high fluxes of penetrating radiation. In order to minimize the risk of damage to circuits from IESD, the JADE Intra-instrument harness is wrapped with a dissipative kapton tape. In essence, the mission IESD requirements are basically in conflict with the instrument design requirements needed for high voltages in JADE and for spacing between parts at various voltages. In particular, ceramic parts inside JADE posed a significant potential issue leading to considerable collaborative work with the EMI working group to get JADE materials and designs approved.

1.2.3.5 Thermal Environment The Juno spacecraft mission trajectory includes heliocentric distances from $0.87 \mathrm{AU}$ to 5.6 $\mathrm{AU}$, which produce over a factor of 40 difference in solar illumination. Thus, the thermal environment for the JADE instrument is strongly driven by these extreme thermal cases. The JADE limits are shown in Table 2. The sensors are located on the periphery of the spacecraft, have large apertures and therefore have larger temperature extremes. The Ebox is located within the Juno Radiation Vault and therefore it has a narrower environment which tends to be biased warm.

Owing to the large thermal range of environments, there was extensive thermal testing at multiple times during the development Through this testing, we developed and employed numerous strategies to limit the highest temperatures for JADE, including using different coatings on the tops and bottoms of each deflector of the ion sensor and striving to thermally isolate the deflectors on the ion and electron sensors from the rest of each sensor body. None-the less, shortly after launch, we found that the JADE-I sensor temperatures were $\sim 20^{\circ}$ above those predicted by the pre-launch thermal model.

JADE-I neared, but did not exceed, non-operational limits shortly after launch. A tiger team, including representatives from JADE and the Juno mission (systems, spacecraft, thermal, etc.), was formed to address the issue. An updated JADE-I thermal model was created to better match the as-built configuration and this new model has shown good agreement with flight data. The current baseline plan anticipates JADE-I exceeding planned flight and protoflight temperatures at perihelion. A series of thermal and solar illumination tests on the JADE-I engineering model is being carried out prior to perihelion to determine the effect of the thermal environment on JADE-I. After JADE temperatures fall below operational limits post-perihelion, the instrument will be turned on again with additional precautions.

1.2.3.6 Contamination Maintaining cleanliness of the sensors is crucial for long life for the MCPs. Thus, throughout development, the sensors were maintained in clean environ- 
Table 3 Contamination

Fig. 3 Random vibration environment

Table 4 Random vibration spectrum and overall GRMS

\begin{tabular}{ll}
\hline $\begin{array}{l}\text { Contamination budget for JADE } \\
\text { exterior by mission phase }\end{array}$ & $\begin{array}{l}\text { Cleanliness level per } \\
\text { IEST-STD-CC1246D }\end{array}$ \\
\hline Pre-calibration testing & $400 \mathrm{~A} / 5$ \\
Post-environmental testing & $500 \mathrm{~A} / 2$ \\
At ATLO delivery & $500 \mathrm{~A} / 2$ \\
Launch & $500 \mathrm{~A} / 2$ \\
High voltage turn-on & $550 \mathrm{~A}$ \\
EOL & $550 \mathrm{~A}$ \\
\hline
\end{tabular}

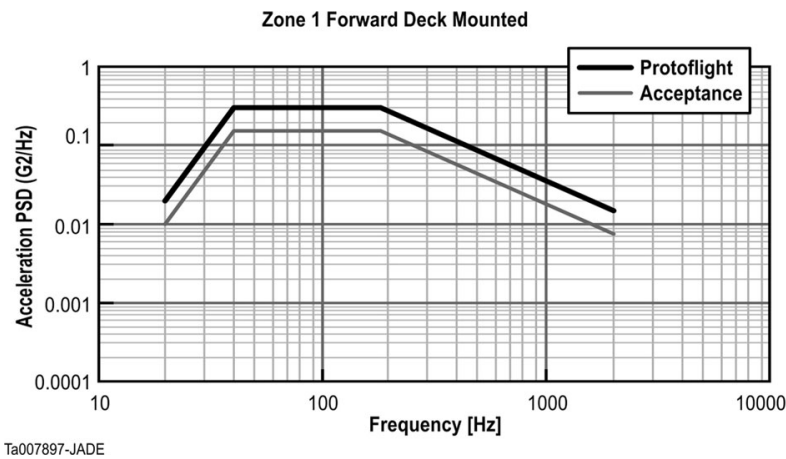

\begin{tabular}{cll}
\hline Freq $(\mathrm{Hz})$ & Protoflight $\left(\mathrm{g}^{2} / \mathrm{Hz}\right)$ & Acceptance $\left(\mathrm{g}^{2} / \mathrm{Hz}\right)$ \\
\hline 20 & 0.02 & 0.01 \\
40 & 0.30 & 0.15 \\
180 & 0.30 & 0.15 \\
2000 & 0.015 & 0.0075 \\
GRMS & 11.9 & 8.4 \\
\hline
\end{tabular}

ments: clean room, clean bagged and purged, or sealed in gaseous $\mathrm{N}_{2}$ backfilled boxes at all times. Tape lifts, visual inspection, and non-volatile residue (NVR) rinses were performed during the development of JADE to ensure that it met all required external cleanliness levels (documented in Table 3).

1.2.3.7 Vibration The Juno launch environment is similar to other missions launched on an Atlas V launch vehicle. However, the JADE sensors carry radiation shielding that is much heavier than typical spaceflight instruments, which are not operated in such harsh radiation environments. Thus, we developed a program of vibration testing starting at the EM level that provided insights into this unique development case and allowed us to find and solve vibration induced structural issues with the heavy radiation shields early in the program. Figure 3 and Table 4 provide the random vibration environment requirements for JADE. 
Fig. 4 Photograph of flight JADE-I (left) and JADE-Es (right) in the clean room, just prior to spacecraft delivery. Two of the JADE-Es (rear) have their protective "red-tag" covers on, while one (front right) and JADE-I have theirs off to show the open, gridded apertures

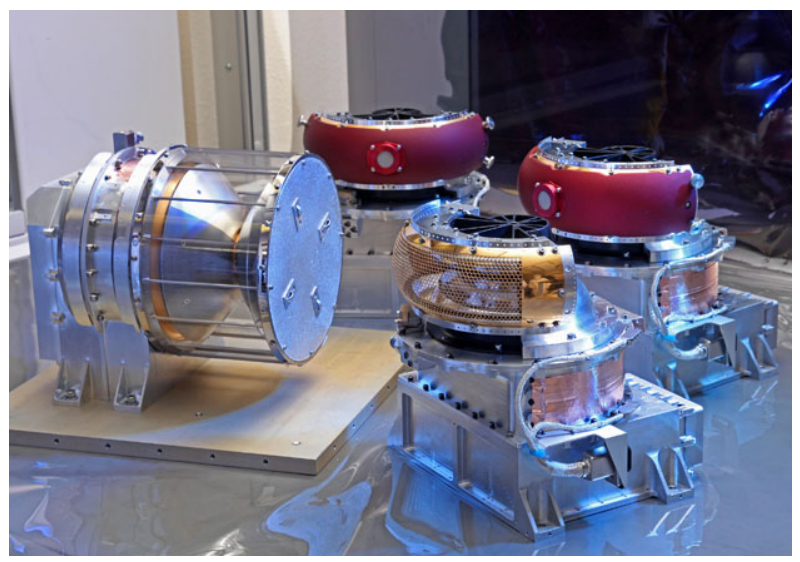

Fig. 5 Placement and FOVs of JADE-I and the three JADE-Es on the Juno spacecraft

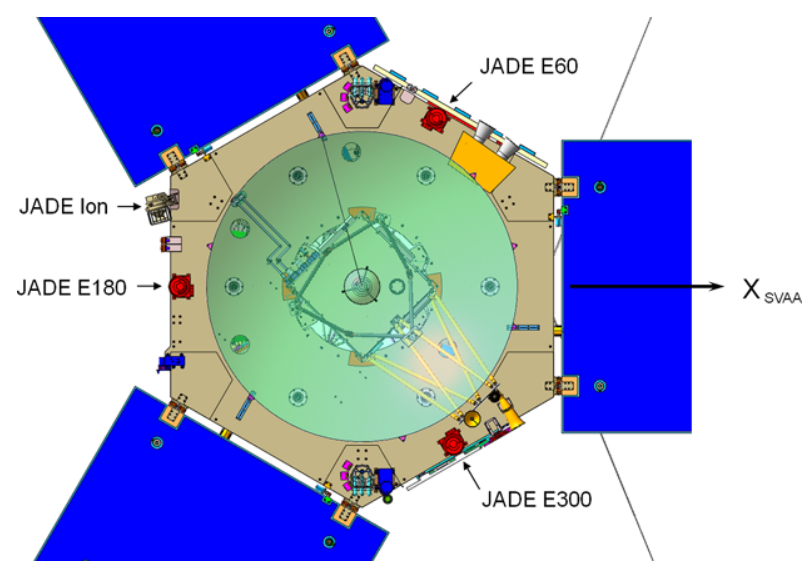

\section{JADE Instrument Description}

\subsection{Overview}

The JADE instrument suite comprises three identical electron sensors (JADE-Es), an ion composition sensor (JADE-I), and a common electronics unit (EBox) that services all four sensors. The four flight sensors are shown, just prior to spacecraft delivery, in Fig. 4, where JADE-I (left) and one of the three JADE-Es (front) have their protective "red-tag" or Remove Before Flight covers removed to expose their entrance apertures. Figure 5 shows the placement and FOVs of the four sensors on the Juno spacecraft. Note that the three JADE-Es instantaneously view all spin phases (angle in the plane of this image) and thus can make detailed electron pitch-angle distribution measurements at very high time cadence, independent of spacecraft spin. JADE-I, in contrast, views primarily perpendicular to this plane and sweeps out all angles each $30 \mathrm{~s}$ spin. 
Fig. 6 Schematic diagram of the JADE-E sensor

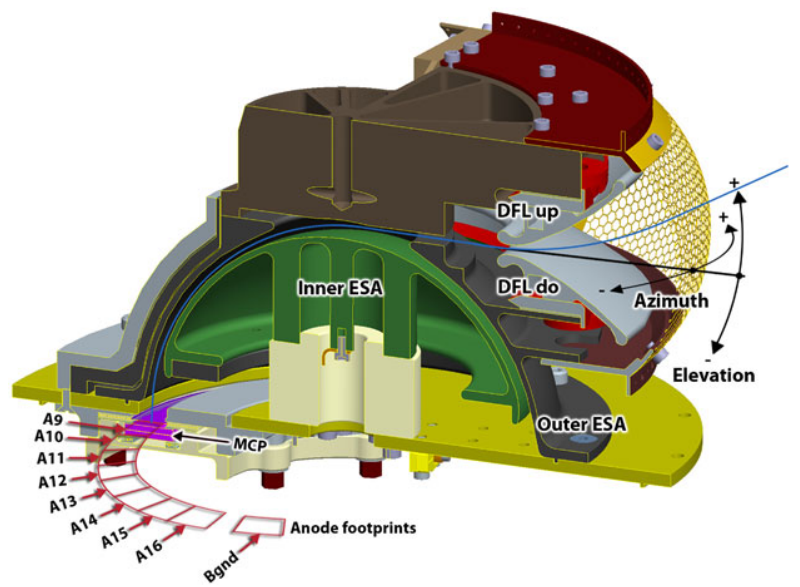

Table 5 JADE-E performance summary

\begin{tabular}{|c|c|c|}
\hline Quantity & Performance & Notes \\
\hline Total mass $[\mathrm{kg}]$ & 5.240 & $\begin{array}{l}\text { Per sensor } \\
\text { Includes radiation shielding }\end{array}$ \\
\hline Total power [W] & 1 & Per sensor \\
\hline Overall dimension $[\mathrm{cm}]$ & $21 \times 21 \times 21$ & Per sensor \\
\hline Energy range $[\mathrm{keV}]$ & 0.1 to 95 & $\begin{array}{l}\text { Upper limit by analysis (tested up to } \\
30 \mathrm{keV} \text { ) }\end{array}$ \\
\hline Energy resolution $[\%]$ & 10.4 to 13.2 & $\begin{array}{l}\text { At zero elevation angle between } 0.2 \text { and } \\
40 \mathrm{keV} \text {, cf. Table } 11\end{array}$ \\
\hline $\begin{array}{l}\text { Geometric factor } \times \varepsilon \\
{\left[\mathrm{cm}^{2} \mathrm{sr} \mathrm{eV} / \mathrm{eV}\right]}\end{array}$ & $\sim 2$ to $5 \times 10^{-5}$ & $\begin{array}{l}\text { Measured values convolved with detector } \\
\text { efficiencies, cf. Sect. 3.2.1.3 }\end{array}$ \\
\hline Analyzer constant $[\mathrm{eV} / \mathrm{V}]$ & 9.095 to 9.182 & At $10 \mathrm{keV}$ for E060, cf. Table 9 \\
\hline Pixel (azimuth $\times$ elevation) & $7.5^{\circ} \times 3-6^{\circ}$ & Elevation angle dependent \\
\hline FOV (azimuth $\times$ elevation) & $360^{\circ} \times \pm 35^{\circ}$ & $\sim 16^{\circ}$ for $100 \mathrm{keV}$, up to $30^{\circ}$ for $<50 \mathrm{keV}$ \\
\hline
\end{tabular}

\subsection{JADE-E}

\subsubsection{Description}

JADE-E consists of three identical sensors that measure the pitch-angle distribution of electrons from $\sim 0.1$ to $\sim 100 \mathrm{keV}$ with a spherical top-hat electrostatic analyzer (ESA), two deflectors, and an MCP detector with an anode ring underneath. A schematic cross section and performance summary are provided below (Fig. 6 and Table 5, respectively).

2.2.1.1 Electro-Optics Design The azimuth angle (the angle in the imaging plane of the analyzer-see Fig. 6), is determined by measuring the position where electrons impact the detector with a position-sensitive anode. The elevation angle, which defines the incoming direction of electrons, is measured perpendicular to the imaging plane, with positive angles defined when the electron comes from above the imaging plane. Each sensor has an FOV of $120^{\circ}$ and all three are mounted such that they cover $360^{\circ}$ in the imaging plane (Fig. 5). The 
spacecraft $X$ axis is defined by azimuth and elevation of $\left(0^{\circ}, 0^{\circ}\right)$, spacecraft $Y$ by $\left(90^{\circ}, 0^{\circ}\right)$, and spacecraft $Z$ by (any azimuth, $90^{\circ}$ ).

The deflectors (upper, DFL-UP, and lower, DFL-DN) provide an energy-dependent deflection of electron trajectories up to about $\pm 35^{\circ}$ before the electrons enter the energy analyzer. The deflectors are biased alternately with positive voltages up to $+10 \mathrm{kV}$. The inner ESA electrode (Fig. 6, blue) is also biased up to $+10 \mathrm{kV}$. There is a high transmission ( $\sim 90 \%$ ), electroformed nickel grid (not shown) on top of the MCP stack (purple). The MCP detector is biased at a voltage that ensures saturated amplification in the MCPS (can be set up to $+3.8 \mathrm{kV}$ maximum power supply). All the other parts are at ground potential.

The ESA has three functions: to (1) select the energy of the electrons; (2) focus electrons onto the imaging plane for the azimuthal direction measurement; and (3) help attenuate the UV radiation to which MCPs are sensitive. The deflectors select the look direction (elevation angle) with respect to the imaging plane. The following criteria drove the ESA and the deflector designs:

- Measure electrons up to $\sim 100 \mathrm{keV}$ using voltages up to a maximum of $+10 \mathrm{kV}$;

- Provide required angular and energy resolutions;

- Limit the electric field inside the sensor to a maximum of $\sim 4 \mathrm{kV} / \mathrm{mm}$ (approximated by the potential difference divided by the minimum distance between electrodes);

- Require only positive-bias high voltages for the electro-optics; and

- Use a spherical top-hat ESA to keep the sensor as compact as possible.

Our design strategy started with an ESA radius of curvature of $\sim 50 \mathrm{~mm}$ and a gap of $2.5 \mathrm{~mm}$. Then we used an automated computer optimization system (optimizer) to refine the design for maximum performance within the constraints listed above. The optimizer created and evaluated different geometries using the electro-optics simulation software SIMION ${ }^{\circledR}$ (Dahl 2000). SIMION simulates a design then returns a certain value (determined by a function that can be the throughput, for example) to the optimizer that compares the performance with previous designs. For JADE-E, the optimizer worked within a parameter space defined by the user and explored the effects of varying the parameters. Using a simplex algorithm, the optimizer adjusted the parameters until it found a local minimum. We changed the initial conditions and modified the parameter space and repeated many optimizations until we found a design that gave excellent results. The criteria for the ESA optimization were to maximize the throughput and angular resolution in the imaging plane (i.e., these parameters were used in the function for the optimization) while keeping the energy resolution $(\Delta E / E$ FWHM) around $10 \%$ of the central energy of the ESA passband. Next, we used the optimizer on the deflectors to redirect $40 \mathrm{keV}$ electrons up to $\sim 45^{\circ}$ with $+10 \mathrm{kV}$ maximum. The criteria for optimizing the deflectors were to keep the FWHM of the angular distribution to better than $5^{\circ}$ and to maximize the throughput. The electric field constraint led us to round all edges.

All of the optical surfaces inside the top-hat analyzer (except the deflectors) were blackened using the Ebonol-C process to significantly reduce the UV transmission over bare aluminum (Al) surfaces (e.g., Zurbuchen et al. 1995).

A schematic view of the detector and the anodes is shown in Fig. 7. The detector consists of (1) a secondary electron suppressor grid (SE Sup); (2) an MCP stack (MCPs and resistor divider); and (3) sixteen $7.5^{\circ}$ (total of $120^{\circ}$ ) adjacent discrete imaging anodes and one $7.5^{\circ}$ background anode. The secondary electron suppressor grid (at $-12 \mathrm{~V}$ ) faces the ESA and repels low energy $(\sim 2 \mathrm{eV})$ secondary electrons that are emitted in this region. The lowest energy $(\sim 100 \mathrm{eV})$ electrons transmitted through the ESA can easily overcome this potential barrier and reach the MCP below the grid. 
Fig. 7 Schematic of the JADE-E detector. A cross section is shown on the right-hand side and a view of the imaging anodes and the background anode on the left-hand side

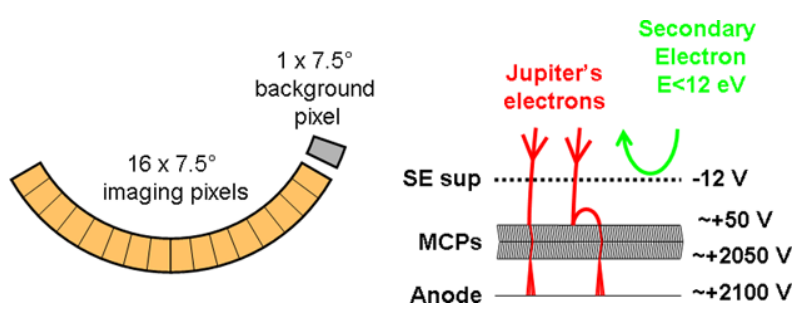

We used Photonis MCPs in a Chevron configuration in series with resistors to provide an accelerating potential $(\sim 50$ to $100 \mathrm{~V})$ between the secondary electron suppressor grid and the top of the MCPs, and between the bottom of the MCPs and the anodes. The MCPs have a low resistance ( $~ 25$ to $30 \mathrm{M} \Omega$ per stack at room temperature), an extended dynamic range, $25 \mu \mathrm{m}$ pore diameter, and a $60: 1$ ratio of length to diameter of pore. Since the anodes are at high voltage, the signal is decoupled to ground with high-voltage capacitors.

Each discrete anode has a dedicated pre-amplifier (A121 from Amptek, threshold set at $\sim 3 \times 10^{6}$ electrons) to register the events. The background anode is identical to the imaging anodes (MCP, anode, decoupling capacitor, pre-amplifier), but its access from the ESA is blocked by a mask built in the secondary electron suppression grid. No electron from the ESA side can reach the top of the MCP stack. Therefore, only penetrating radiation and internal MCP noise can generate a signal on the background anode.

\subsubsection{Influence of a Strong Magnetic Field on the Response In principle, measurement} of the flux as a function of energy and angle to the magnetic field vector is all that is required to determine the pitch-angle distributions of electrons. In high-rate science and burst mode (Sect. 4.2) JADE-E sets the deflector voltage to measure electrons that travel along the magnetic field line. Those electrons fall onto one particular anode in one of the JADEE sensors. The neighboring anodes measure the flux away from the magnetic field vector allowing reconstruction of the pitch-angle distribution.

The ESAs sweep voltages in steps to measure electrons over the JADE-E energy range. For each ESA energy step, the deflection voltage is calculated so that the JADE-E look direction always tracks the magnetic field vector direction. The magnetic field vector components are measured and broadcast to JADE (and other instruments onboard) nominally once per second, with a resolution of $1 \mathrm{nT}$. The vector orientation is calculated and a table of deflection voltages is created for the next energy sweep. If the deflection angle falls outside the $\pm 35^{\circ} \mathrm{JADE}-\mathrm{E}$ FOV, a limit is set to $35^{\circ}$ and the voltages are calculated accordingly. Note that over the Juno mission the spacecraft spin axis orientation and orbit does not require JADE-E to deflect more than $\pm 30^{\circ}$ to track the magnetic field direction. If the voltage required to track the field direction exceeds $10 \mathrm{kV}$, e.g. at the upper end of the energy range and for large deflections, then the voltage is set at $10 \mathrm{kV}$. If the magnetic field vector information is missing or comes late for the next DFL angle calculation, then JADE software extrapolates (propagates) the next orientation using the latest value received. JADE extrapolates up to a configurable number of seconds (currently $25 \mathrm{~s}$ ), and if the information is still missing, JADE-E stops deflecting until a new value is received.

At any time, the magnetic field azimuth is aligned with two anodes of the JADE-E sensors. If the elevation is positive for one of these, then it must be negative for the other, i.e. the first sensor deflects for a positive elevation, and the other for an equally negative elevation. The third sensor does not deflect. For a constant magnetic field vector direction, the configuration of deflecting and non-deflecting sensors changes every sixth of a spacecraft rotation, i.e. every $60^{\circ}$ or $5 \mathrm{~s}$. 
Fig. 8 Histograms of the estimated magnetic field strength that Juno is expected to encounter in the auroral regions

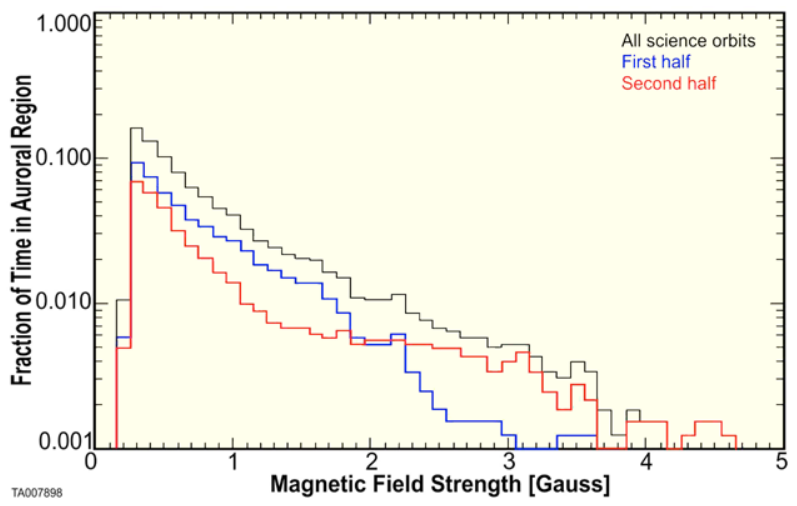

During the course of the mission, Juno traverses auroral regions where the magnetic field strength exceeds $3 \mathrm{G}$. Under these conditions, the gyroradii of low-energy $(<5 \mathrm{keV})$ electrons are comparable to the scale size of the ESA; consequently, measurements from the ESA-based sensor are significantly aberrated. For instance, using the simple relation

$$
r_{L}=(3.37 \mathrm{~cm})(1 \text { Gauss } / B)(E / 1 \mathrm{eV})^{1 / 2}
$$

where $r_{L}$ and $E$ are the electron gyroradius (in $\mathrm{cm}$ ) and energy (in $\mathrm{eV}$ ), a $100 \mathrm{eV}$ electron in a $3.37 \mathrm{G}$ field has a gyroradius of $10 \mathrm{~cm}$.

Figure 8 shows histograms of the estimated magnetic field strength integrated over Juno's baseline trajectory. We used the VIP4 Magnetic Field model that also includes a current sheet model (Connerney et al. 1981, 1998) to calculate the magnetic field strength at the expected location of the spacecraft at 1-min intervals. We then used the same model to trace these field lines to the magnetic equator, here defined as the $L$-shell or the point at which the radial component of the magnetic field vanishes (i.e., $B_{r}=0$ ). We identified a total of 3257 minutes during the mission when the spacecraft is expected to be inside the auroral regions, which we define as regions when $R<3 R_{J}$ and $L>15$. Note that $L=15$ corresponds to the $L$-shell of Ganymede, which has an auroral spot equatorward of the main oval. The peak field calculated in the auroral region is $7.73 \mathrm{G}$. Overall, Juno is expected to be in regions where $B<1 \mathrm{G}$ for $63.4 \%$ of the time, $B<3 \mathrm{G}$ for $95.6 \%$ of the time, and $B<5 \mathrm{G}$ for $99.2 \%$ of the time.

With the aid of detailed SIMION simulations, we characterized and quantified the response of the JADE-E sensor from $200 \mathrm{eV}$ to $40 \mathrm{keV}$ electrons in a wide variety of magnetic field strengths and orientations. Figure 9 shows simulations of pitch-angle deflections that $1 \mathrm{keV}$ and $5 \mathrm{keV}$ electrons undergo after passing through a SIMION model of the JADE-E sensor in an external magnetic field strength of $3 \mathrm{G}$ (which Juno is expected to experience only toward the end of its prime mission). In the upper panel, the crosses represent the pitch-angle mapping done with the JADE-E anodes, and the white line indicates where the crosses would be expected to fall if the magnetic field had no influence on the electron trajectories inside the ESA; the bottom panel shows the difference. It is clear that the degree of pitch-angle distortion depends on the electron energy. For instance, the pitch angles for $1 \mathrm{keV}$ electrons shift by less than $\sim 10^{\circ}$, while pitch angles for $5 \mathrm{keV}$ electrons shift by less than $\sim 5^{\circ}$.

Pitch angle is not the only quantity altered in the JADE-E measurements. The energy measurement and the geometric factor $(G)$ are also affected by the magnetic field. To evalu- 


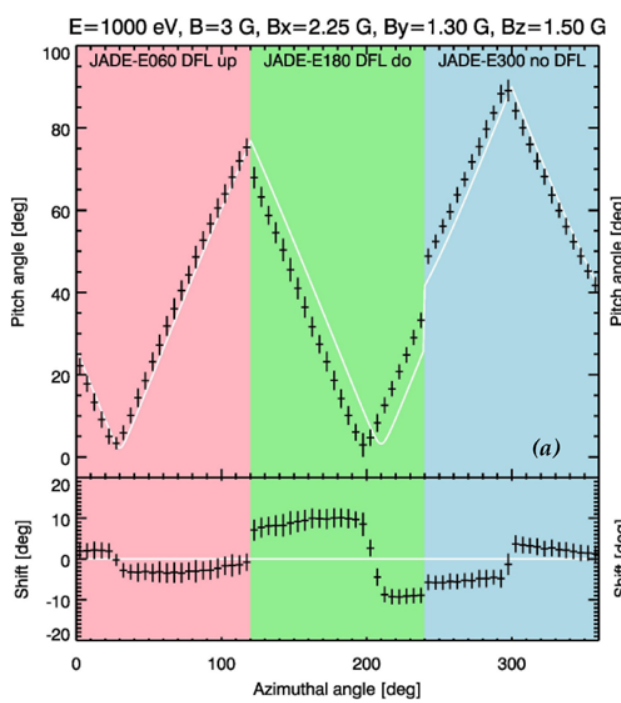

$\mathrm{E}=5000 \mathrm{eV}, \mathrm{B}=3 \mathrm{G}, \mathrm{Bx}=2.25 \mathrm{G}, \mathrm{By}=1.30 \mathrm{G}, \mathrm{Bz}=1.50 \mathrm{G}$

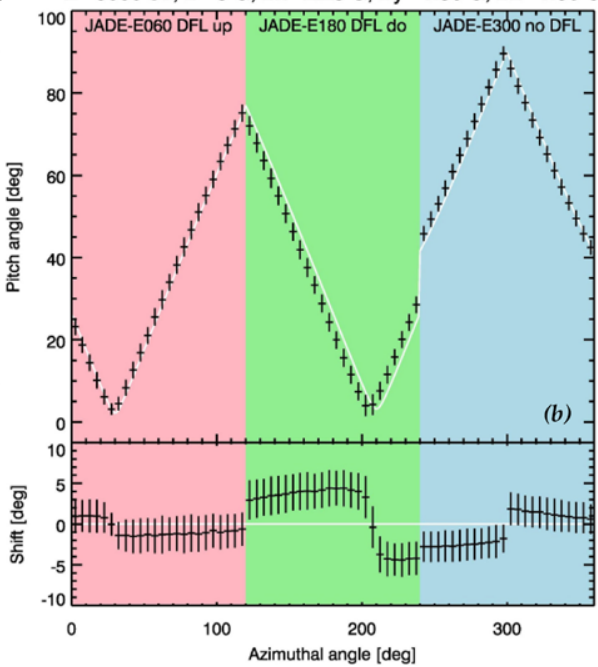

Fig. 9 Simulations of pitch-angle deflection of (a) $1 \mathrm{keV}$ and (b) $5 \mathrm{keV}$ electrons after passing through the ESA of the JADE-E sensor in an external magnetic field strength of $B_{X}=2.25 \mathrm{G}, B_{Y}=1.3 \mathrm{G}, B_{Z}=1.5$, and $B=3 \mathrm{G}$. The white curve is undeflected pitch angle. The bottom panels show the angular shift

ate these other effects of the magnetic field on the JADE-E measurements, we used electrooptics simulations with constant magnetic field. As expected, the strongest effects are seen when the field is strongest and the energy the lowest. In our continuing efforts in preparation for the JADE data analysis phase, we are in the process of simulating a large number of cases of different magnetic field strengths and orientations, electron energies, and look directions to construct a simulation-based forward model for JADE-E. This model will be validated using laboratory measurements with a high-fidelity flight-like unit. The model inputs are the magnetic field vector (i.e., strength and direction) and the electron energy and angle distributions; the output is count rate as a function of anode and energy.

Finally, we note that early in the development of JADE, we also investigated the possibility of using magnetic shielding (mu-metal) around the sensor in order to mitigate the effects of the magnetic field on the electron trajectories. The main result of that study is that although the field inside the sensor is nearly canceled, the field near the aperture is concentrated, creating a strong lens effect on the electrons entering the sensor. Consequently, the response is very difficult to characterize and too complicated to deconvolve from the measurement signal. Therefore, we did not use magnetic shielding.

\subsection{JADE-I}

\subsubsection{Sensor Description}

The JADE-I sensor is a spherical top-hat ESA designed to measure the Jovian magnetospheric ion plasmas. Ions are measured in the energy range from $10 \mathrm{eV} / \mathrm{q}$ to $45 \mathrm{keV} / \mathrm{q}$ for masses that range from 1 to $>40 \mathrm{amu}$. The sensor is mounted on the spacecraft such that the instantaneous, undeflected FOV is $270^{\circ}$ in elevation by $9^{\circ}$ in azimuth. The FOV is in a plane offset $-15^{\circ}$ from the spacecraft $x$-axis (in the $x-y$ plane) and through its $z$-axis (nominally sunward). See Fig. 5 for the mounting configuration of JADE-I on the spacecraft. 
Fig. 10 Cross section of the JADE-I electro-optics
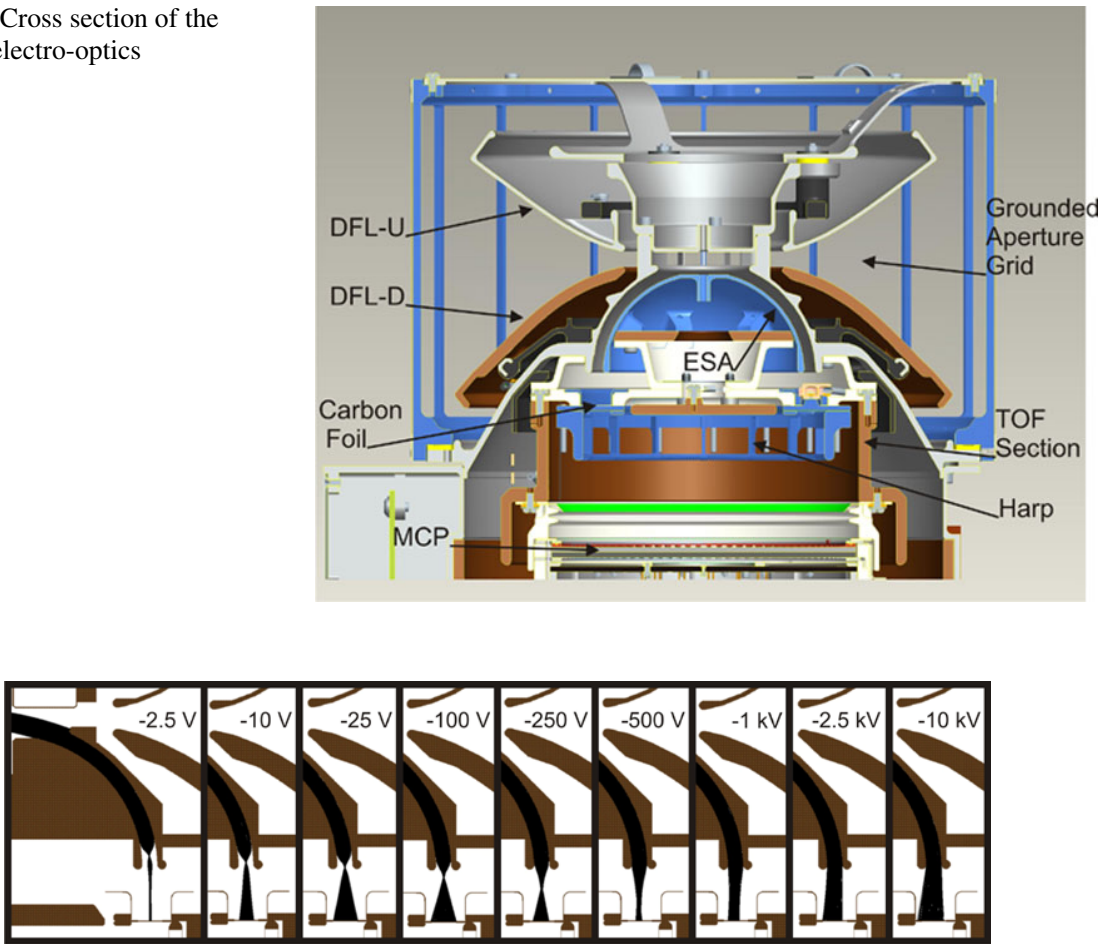

Fig. 11 Simulated exit trajectories for ions passing through the JADE-I ESA. The ESA voltage is varied a range of values, from $-2.5 \mathrm{~V}$ to $-10 \mathrm{kV}$. The carbon foil is at $-10 \mathrm{kV}$ and deflectors are at $0 \mathrm{~V}$ for all these runs

As with JADE-E, incident particles first pass through a set of electrostatic deflectors used to sweep the look direction up to $\pm 45^{\circ}$. Because JADE-I is oriented on the spacecraft with its symmetry axis perpendicular to the spacecraft spin axis, deflection of equatorial ions occurs in spacecraft azimuth rather than elevation. Ions then pass through a $90^{\circ}$ spherical section ESA used to select the energy per charge $(E / q)$ of positive ions. To reduce background from forward scattered UV photons, the outer ESA dome is blackened using Ebanol-C. The inner ESA dome is nickel plated titanium. The whole sensor aperture is covered by a grounded grid with varying line spacing to keep transmission near constant for all incident angles. The effective transmission of the grid is $\sim 86 \%$. See Fig. 10 for a cross-sectional view of the electro-optics.

Ions that pass through the ESA are accelerated by $10 \mathrm{kV}$ into the time-of-flight (TOF) section. A large acceleration past the ESA causes its focal point to move. For the lowest operational ESA voltages $(\sim-2.5 \mathrm{~V})$ the electric fields from the carbon foil can reach a significant distance into the ESA. The penetrating electric field effectively shortens the analyzer by pulling out particles that would ordinarily hit the end of the ESA.

The effect of the post acceleration on the focal point is shown in Fig. 11, as simulated with SIMION. For each panel, a parallel, radial proton beam $(\Delta E / E=30 \%)$ enters the JADE-I sensor with a normal trajectory to the sensor axis. As the energy (ESA voltage) is increased, the focal point moves downward, towards the TOF section, resulting in an angular spread at the carbon foil that also varies with ESA voltage. 
Fig. 12 JADE-I anode layout (front side with back side overlay)

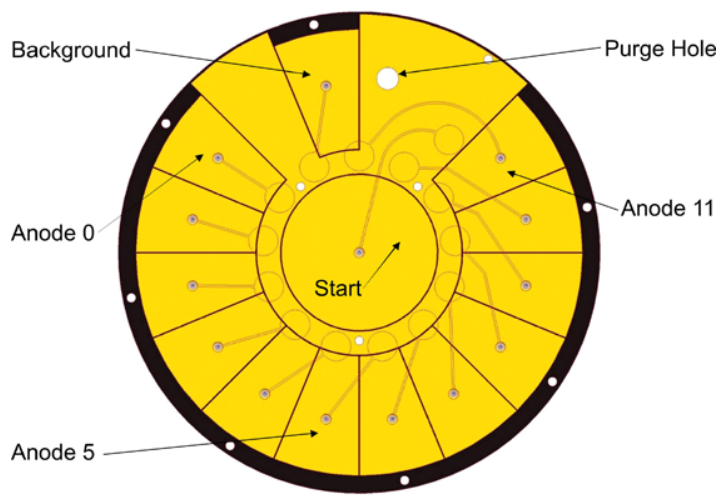

Because the ions receive additional acceleration from the TOF section as they exit from the ESA, the JADE-I analyzer constant has a weak energy dependence (e.g., Randol et al. 2012). Across the full energy range of the sensor ( $10 \mathrm{eV}$ to $45 \mathrm{keV})$, the analyzer constant varies by $\sim 1.3 \%$. At lower energies the ESA has a larger $\Delta E / E$, broader azimuthal angular resolution and narrower elevation angular resolution, with the energy resolution varying from nearly $30 \%$ at the lowest energy step to $\sim 19 \%$ at the highest. The larger $\Delta E / E$ and angular width also lead to a larger geometric factor for the lower energies. The energy dependent response of the analyzer is discussed further in Sect. 3.3.

After exiting the ESA, an ion passes through an ultra-thin carbon foil (McComas et al. 2004), which emits secondary electrons. The secondary electrons are accelerated to $\sim 8 \mathrm{keV}$ toward the center of the MCP where they are detected as a start pulse. The ion is detected on the perimeter of the MCP as a stop pulse on one of twelve, $22.5^{\circ}$ wide anodes, providing the elevation of the incident particle. (Because of the way JADE-I is mounted on the spacecraft, imaging is in the elevation direction, as opposed to JADE-E where imaging in the azimuthal direction.) The charge state of the ion exiting the foil depends on the particle's energy and species. However, for the JADE-I energy range, the ions leave the carbon foil with a predominantly neutral charge state. A 13th anode, with a FOV obscured by internal structure, is used to measure the background rate. Figure 12 shows the layout of the JADE-I anode. The TOF electronics is capable of measuring particles with masses that range from $1 \mathrm{amu}$ (protons) to $>40 \mathrm{amu}$ (Argon) over the full energy range (10 eV to $45 \mathrm{keV}$ ) of the instrument.

The JADE-I sensor uses a Chevron stack of $60: 1$ Phontonis MCPs that are biased so that the front grid is $\sim-100 \mathrm{~V}$ with respect to the front face. The anode is held at ground and the initial MCP voltage setting is $-2100 \mathrm{kV}$. The MCP supply is capable of producing up to $-3.8 \mathrm{kV}$, which allows the MCPs to be returned to saturation if their gain decreases over the mission.

To provide shielding from energetic particles, the wall thickness of the electronics enclosure and electro-optics were tailored to provide maximum protection within a minimum mass. Shielding of the JADE-I and JADE-E sensors is described in Sect. 2.5.

For a summary of the JADE-I performance and other instrument properties, see Table 6. See Sect. 3.3 for a more detailed discussion of JADE-I calibration. 
Table 6 JADE-I performance summary

\begin{tabular}{|c|c|c|}
\hline Quantity & Performance & Notes \\
\hline Total mass $[\mathrm{kg}]$ & 7.552 & Includes radiation shielding \\
\hline Total power [W] & 1.9 & \\
\hline Overall dimension $[\mathrm{cm}]$ & $18 \times 24 \times 22$ & \\
\hline Energy range $[\mathrm{keV}]$ & 0.01 to 46.2 & Lower limit by analysis (Tested down to $1 \mathrm{keV}$ ) \\
\hline Energy resolution $[\%]$ & 28 to 18 & Function of incident energy, cf. Fig. 59 \\
\hline $\begin{array}{l}\mathrm{G} \text { factor } \times \varepsilon \text { w/valid TOF per } \\
\text { pixel }\left[\mathrm{cm}^{2} \mathrm{sreV} / \mathrm{eV}\right]\end{array}$ & $\sim 3$ to $5 \times 10^{-5}$ & $\begin{array}{l}\text { Measured values convolved with detector } \\
\text { efficiencies, cf. Sect. 3.2.1.3 }\end{array}$ \\
\hline $\begin{array}{l}\text { G factor } \times \varepsilon, \text { All_STARTS full } \\
\text { FOV }\left[\mathrm{cm}^{2} \mathrm{sr} \mathrm{eV} / \mathrm{eV}\right]\end{array}$ & $\sim 4$ to $6 \times 10^{-3}$ & $\begin{array}{l}\text { Measured values convolved with detector } \\
\text { efficiencies, cf. Sect. 3.2.1.3 }\end{array}$ \\
\hline Analyzer constant $[\mathrm{eV} / \mathrm{V}]$ & 4.62 & \\
\hline Pixel (azimuth $\times$ elevation) & $8.5^{\circ} \times 30^{\circ}$ & Energy dependent, cf. Sect. 3.3.1 \\
\hline FOV (azimuth $\times$ elevation) & $\pm 45^{\circ} \times 270^{\circ}$ & $\pm 45^{\circ}$ for up to $24 \mathrm{keV}$, up to $\pm 25^{\circ}$ at $40 \mathrm{keV}$ \\
\hline Mass range & $1->50 \mathrm{amu}$ & Tested up to $40 \mathrm{amu}(\mathrm{Ar}+)$ \\
\hline Mass resolution & $2.5->11$ & Mass and energy dependent, cf. Fig. 67 \\
\hline
\end{tabular}

\subsection{Electronics Architecture}

\subsubsection{Overview}

The high radiation environment for the Juno mission drives the architecture of the JADE electronics. The majority of the electronics are housed in the Juno Radiation Vault to minimize system resources. The JADE-E and JADE-I sensors are distributed on the periphery of the payload deck to achieve the FOV required for the science measurement. The front-end electronics in the sensors are minimized to limit the required radiation shielding mass. An Intra-Instrument Harness connects each of the JADE-E and JADE-I sensors to the JADE Electronics Box in the Juno Radiation Vault where high voltage for the detector and electrooptics is generated and the sensor signals are processed. Figure 13 shows the block diagram of the JADE electronics (board dimensions are summarized in Table 8).

\subsubsection{JADE Electron Sensor Electronics}

The JADE-E Sensor Electronics is made up of five separate boards. The Anode Board collects charge from the output of the MCPs. Because the anode is at high voltage, the Capacitor Board is required to bring the signal levels down to ground potential and incorporates "zap-traps" to protect the inputs of the charge amplifiers. The Charge Amplifier Board processes these charge signals, transforming them into digital pulses. The Digital Board takes the parallel array of Charge Amplifier outputs and serializes them for transmission from the sensor to the electronics box to minimize cabling over the long runs from the three sensors to the radiation vault. The High Voltage Distribution Board (HVDB) filters the incoming high voltage. The block diagram of the JADE Sensor Electronics is shown in Fig. 14.

2.4.2.1 JADE-E Anode The JADE-E Anode (Fig. 15) is a ceramic board that collects charge from the MCPs. It is divided into seventeen $7.5^{\circ}$ segments. Sixteen anodes provide $120^{\circ}$ of imaging for the particles collected through the ESA. The seventeenth anode bin is "dark" and measures the background from penetrating radiation. The ceramic anode 


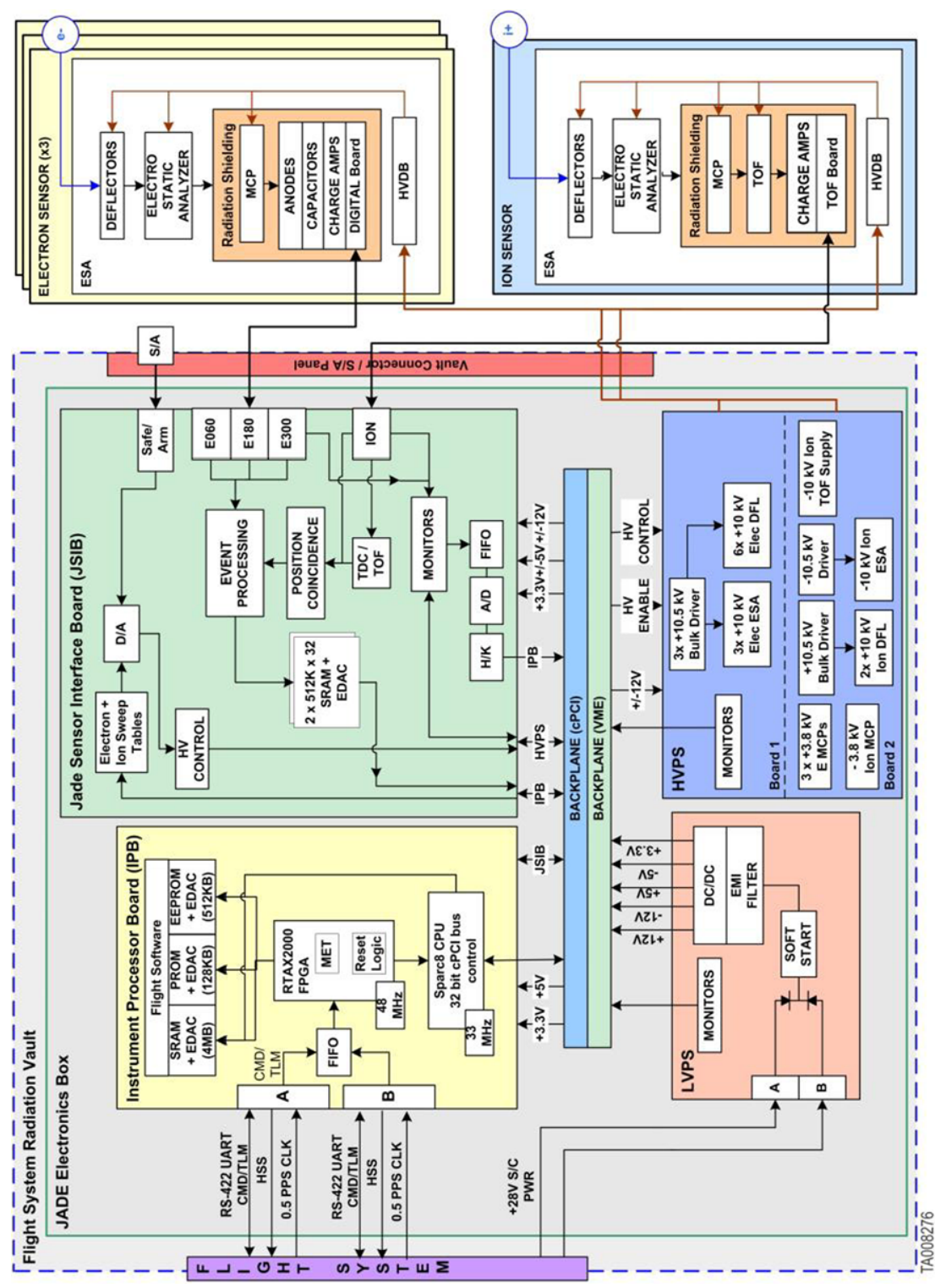

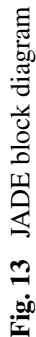




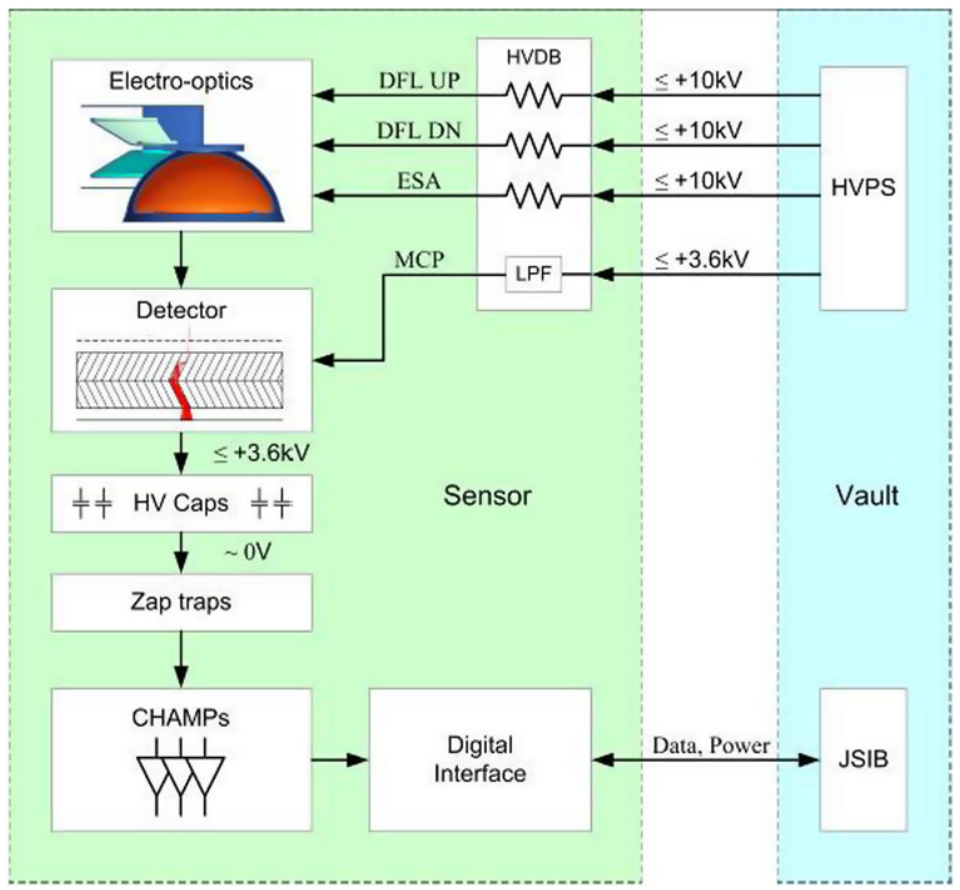

Fig. 14 JADE-E Sensor Electronics block diagram

Fig. 15 JADE-E Anode

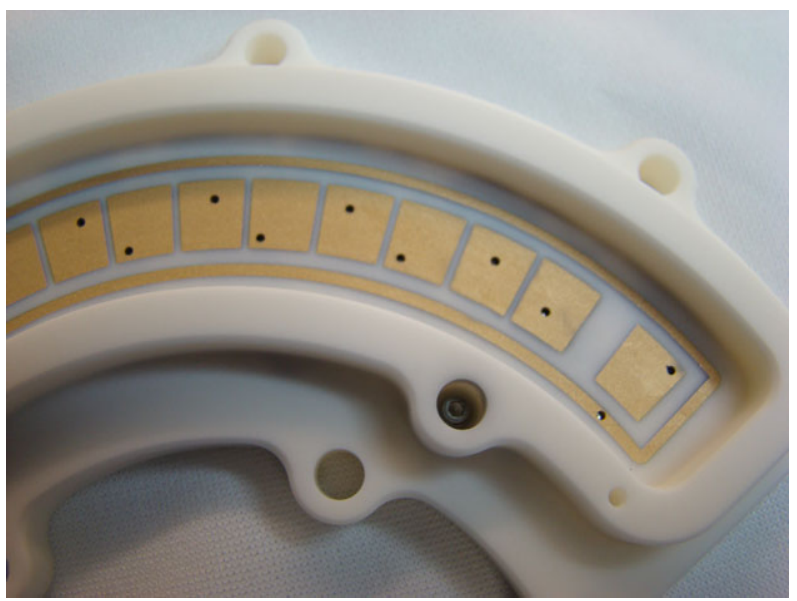

also provides a seal that separates the ultra-high purity detector region, which is limited to glass, metal, and ceramic materials, from the standard electronics boards, which outgas at higher rates and contain polymer materials. The MCPs are stacked into the JADE-E Anode and a pickup ring is provided to make electrical connection to the MCP output face. The anode segments and the output face of the MCP are at high voltage. The ceramic anode has walls that surround the MCPs and provide the high dielectric strength necessary to prevent high-voltage breakdown to the tantalum shielding that is in close proximity to the MCPs. 
Fig. 16 JADE-E Capacitor Board

Fig. 17 JADE-E Charge Amplifier Board
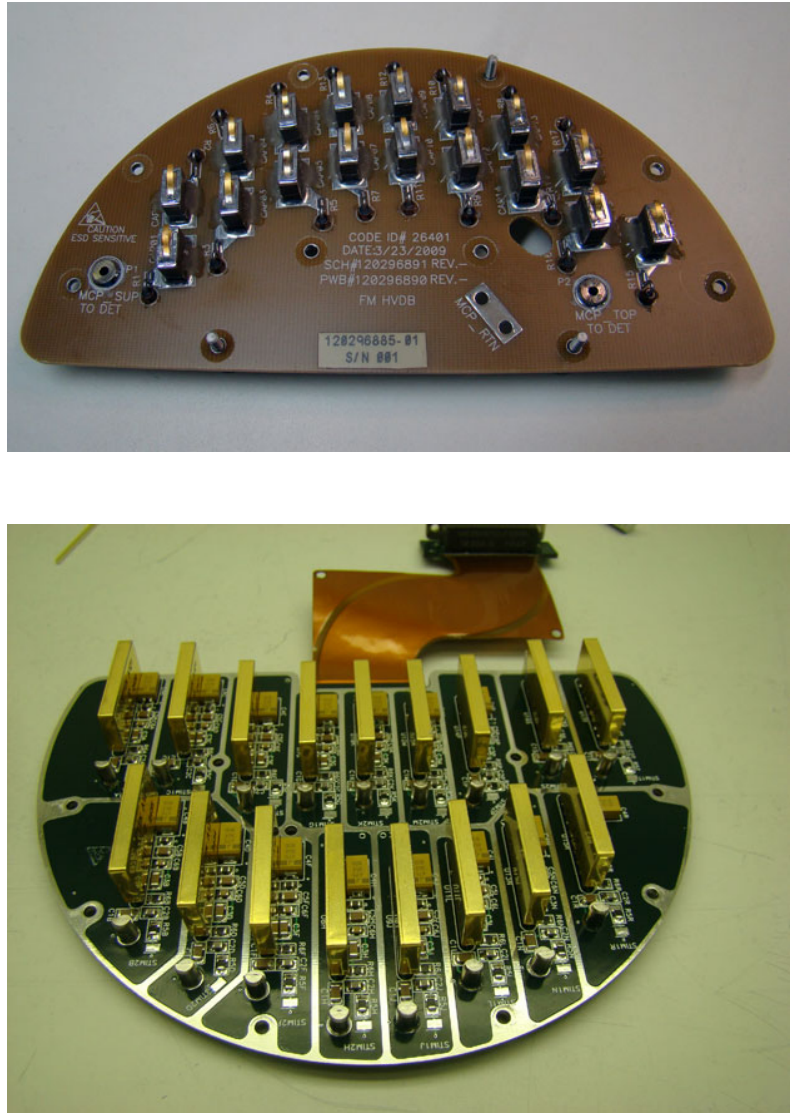

2.4.2.2 Capacitor Board The Capacitor Board (Fig. 16) decouples the high-voltage signals from the anodes down to ground potential where they can be processed by the analog electronics. $1000 \mathrm{pF}$ surface-mount high voltage $(6 \mathrm{kV})$ ceramic capacitors are stood vertically on the board with a solder connection on only the board end in a "tomb-stone" array. A beryllium copper spring is soldered to the top end-cap of the capacitor. When stacked together the springs on the Capacitor Board make electrical contact with the back of the Anode Board to provide the signal path for each of the anodes. The decoupled signals are then transmitted through the board using an axial resistor that is mounted with one wire connected to the top side and one connected to the bottom side of the Capacitor Board. On the bottom side of the board, the signal from the resistor is limited using back-to-back diodes that form a "zap trap". The axial leaded resistor is mounted through the board to minimize board space and eliminate any arc paths around the zap-traps using the circuit board itself as a barrier.

2.4.2.3 Charge Amplifier Board The Charge Amplifier Board (Fig. 17) uses 17 Amptek A121 charge amplifiers to process the MCP pulses and transform them from charge signals into digital signal. Pogo pins are used to bring the signals down from the Capacitor Board to the Charge Amplifier Board. Each channel's processing electronics is isolated from the other channels by a pocketed aluminum housing cover (Fig. 18). Resistors are used to set 
Fig. 18 JADE-E Board stiffener/electro-static isolation housing

Fig. 19 JADE-E Digital Board
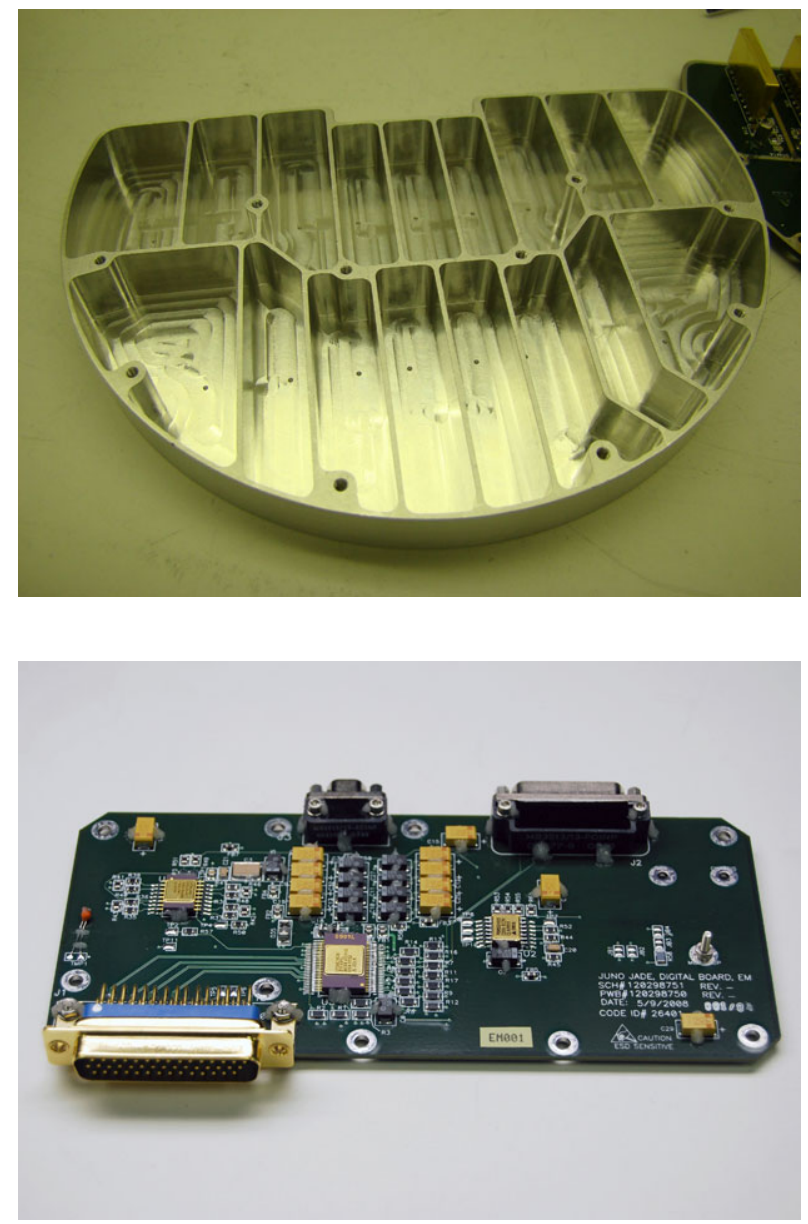

the various adjustable parameters of the A121. The threshold is fixed at $3 \times 10^{6}$ electrons. The pulse width is set to $90 \mathrm{~ns}$ and the dead-time at $180 \mathrm{~ns}$.

2.4.2.4 Digital Board The digital board (Fig. 19) registers digital pulses from all seventeen of the A121 outputs. It uses passive termination to translate the $5 \mathrm{~V}$ level outputs from the A121 to the $3.3 \mathrm{~V}$ serializer input levels. The serializer chip is employed to minimize wiring from the sensor electronics to the JADE Ebox; the signals are serialized into a frame and transmitted through high-speed, low-voltage differential signaling (LVDS) lines to the Sensor Interface Board in the Ebox. The sampling clock frequency, the A121 pulse width, and the A121 dead-time were selected to assure that at least one high-state and one low-state is transmitted serially for each of the inputs so that the JADE Sensor Interface Board (JSIB) can correctly count all pulses of all A121 channels at a periodic rate of $5.55 \mathrm{MHz}$ for each pixel. The serializer chip samples all of the inputs every $60 \mathrm{~ns}$ and, using a phase-locked loop, transmits the samples to the JSIB over three pair of high speed LVDS serial lines. The LVDS signal levels allow us to transmit at high bandwidth while maintaining the low EMI emissions required for compatibility with the MWR instrument. Figure 20 shows the JADE-E digital board timing. 


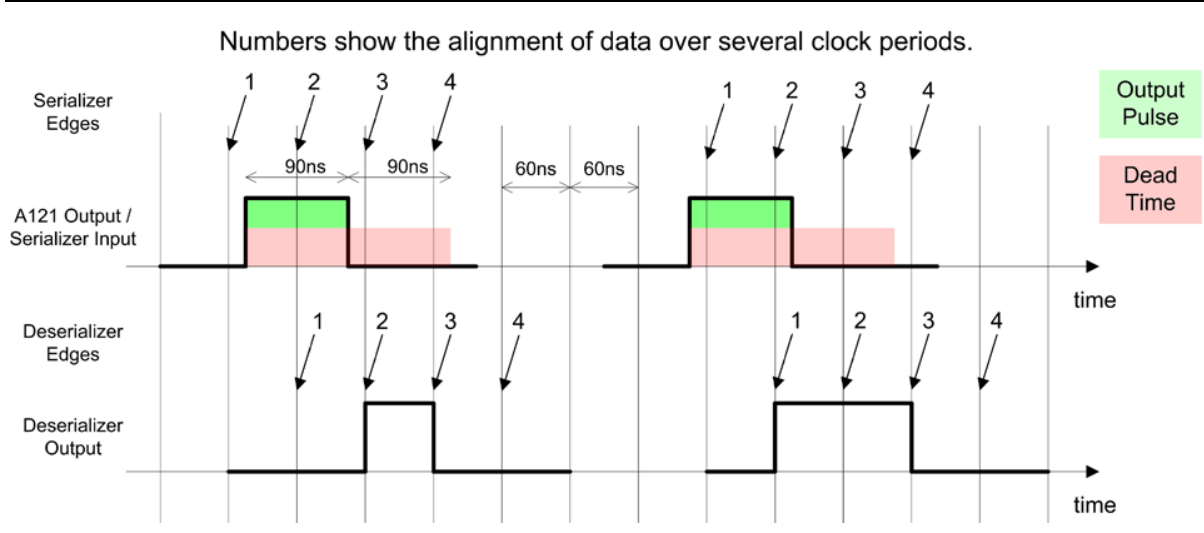

Fig. 20 JADE-E Digital Board timing diagram

Fig. 21 JADE-E High Voltage Distribution Board (HVDB)

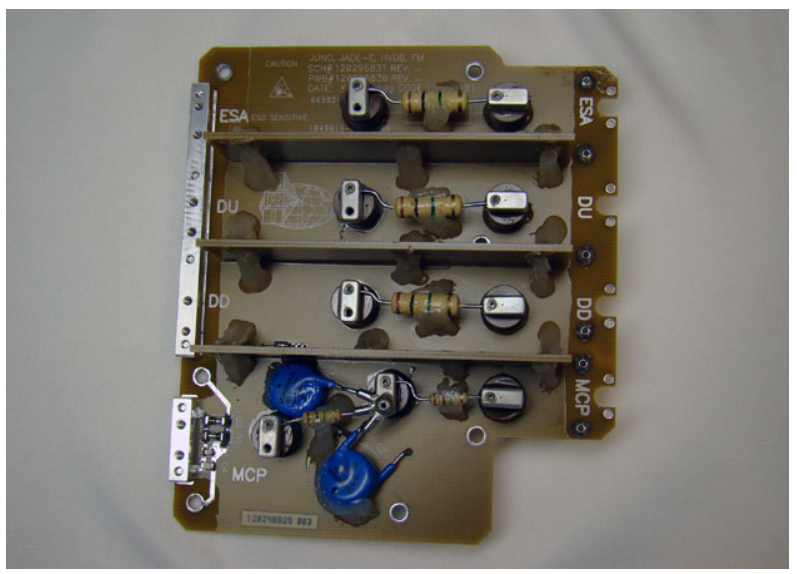

2.4.2.5 High Voltage Distribution Board The High Voltage Distribution Board (HVDB) (Fig. 21) provides shielded, mechanical termination of the doubly-shielded high voltage coaxial cables, filters the detector bias signals, and provides dropping resistors to set the proper optical voltages in the detector. For the optical high voltages within the JADE-E sensor, this HVDB provides a convenient way to make connections between the high voltage wiring within the sensor and the pig-tailed high voltage wiring that comes from the various high voltage power supplies (HVPSs) in the Ebox. The HVDB provides local zap-traps to provide a chassis return path as close as possible to the sensor, should any discharges occur there. The HVDB also provides a 1-pole filter that attenuates the ripple on the MCP supply below 0.1 Vpp peak-to-peak.

\subsubsection{JADE Ion Sensor Electronics}

The JADE-I Sensor Electronics is made up of five separate boards (see block diagrams in Figs. 22 and 23). The Anode Board collects charge from the output of the MCPs. The Time-of-Flight (TOF) Board contains the amplifiers and constant fraction discriminators that create the digital, LVDS Start and Stop signals that the JSIB uses to measure the TOF of each ion. The Charge Amplifier Board measures the pulses from the Stop Anodes to 


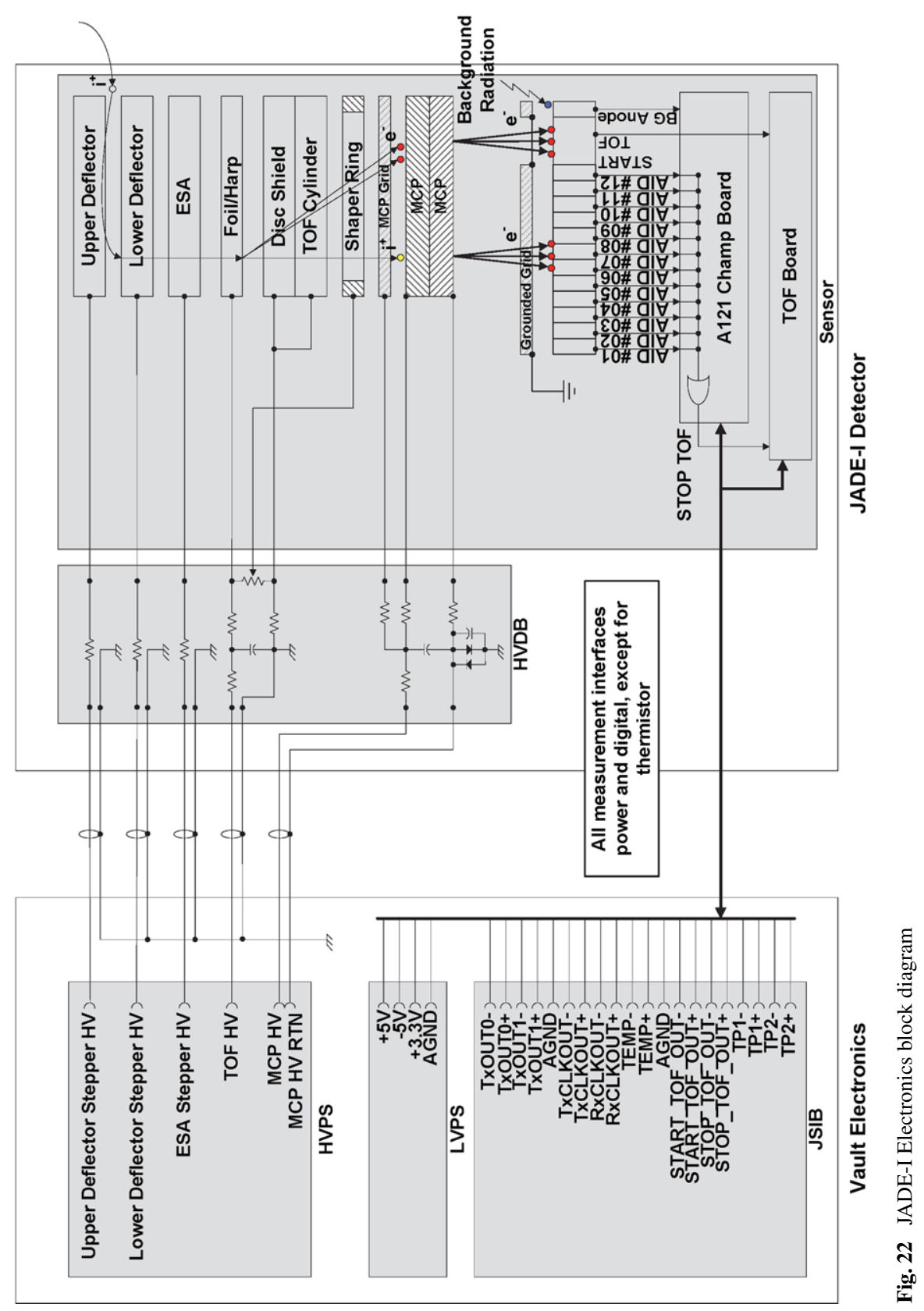




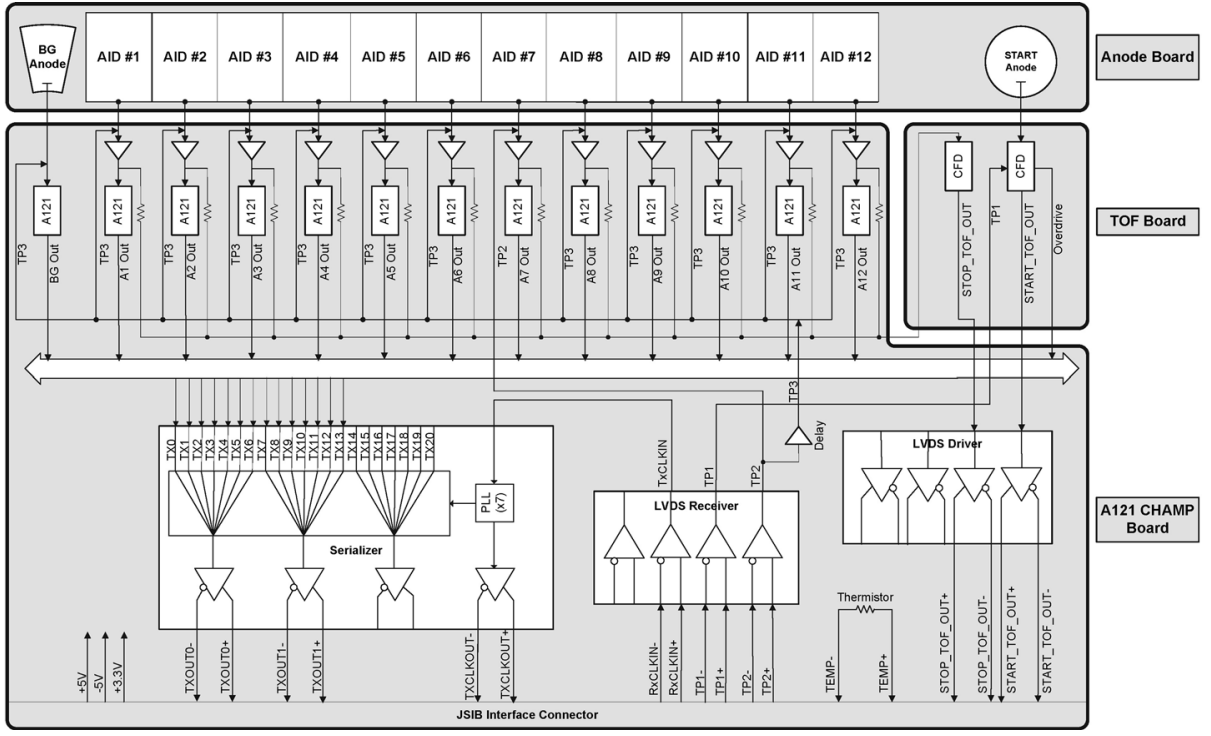

Fig. 23 Overview of the JADE-I Charge Amplifier and TOF Boards

Fig. 24 JADE-I Anode

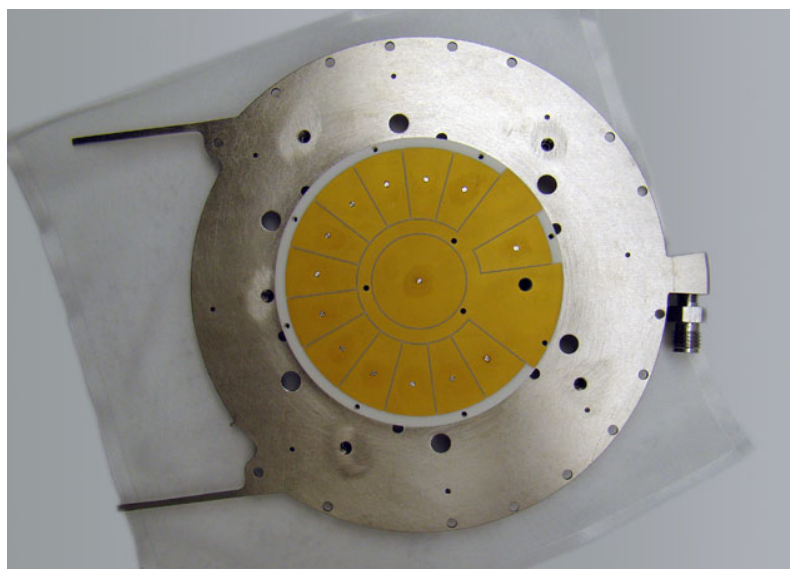

determine the elevation angle of the incoming ion. The Digital Board takes the parallel array of Charge Amplifier outputs and serializes them for transmission from the sensor to the electronics box to minimize the cabling over the long run from the JADE-I sensor to the radiation vault. The HVDB filters the incoming high voltage signals and provides the divider resistors that are used to generate the optical high voltages for the TOF measurement system.

2.4.3.1 JADE-I Anode The JADE-I Anode (Fig. 24) is a ceramic board that collects charge from the MCPs. It is divided into a start anode in the central portion, which measures signals from the portion of the MCP that collects start-electrons from the carbon foils. The remaining outer annulus is divided into thirteen 22.5 degree anode bins. Twelve of the anode bins are used to measure the elevation angle of the incoming ion as well as provide a stop signal for the TOF electronics. The remaining 22.5 degree anode bin is "dark" and used to 
Fig. 25 JADE-I TOF Board

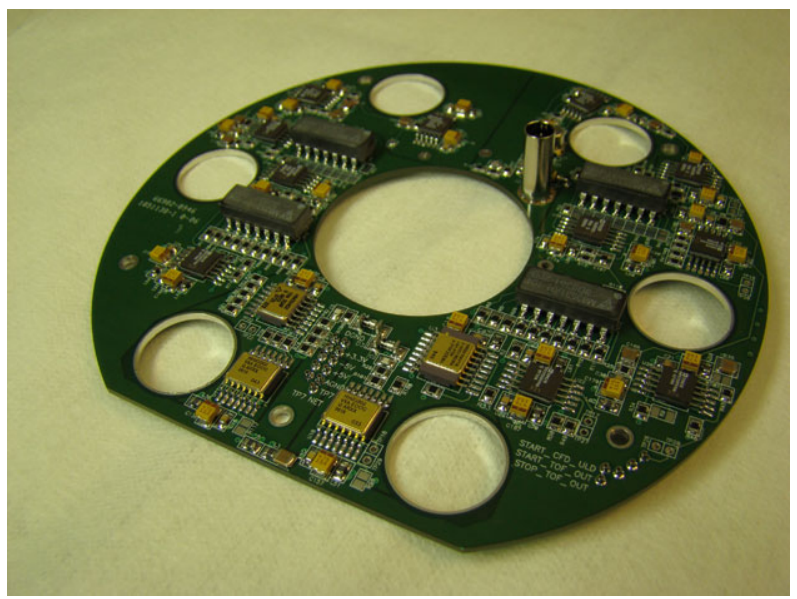

collect background rates for penetrating radiation. Similar to JADE-E, the JADE-I ceramic anode also separates the ultra-high purity detector region from the standard cleanliness in the volume that contains the front-end electronics.

2.4.3.2 Time-of-Flight (TOF) Board The TOF Board (Fig. 25) takes signals from the Start Anode and the sum of all of the anode signals from the Charge Amplifier Board and performs constant-fraction discrimination to convert these analog signals into digital start and stop pulses, which are transmitted to the JSIB in the Ebox over dedicated LVDS signal pairs. The TOF Board includes lower- and upper-level discriminators. The dynamic range is from $1 \times 10^{6}$ to $5 \times 10^{7}$ electrons. When an in-range signal is processed, the lower-level discriminator opens up a comparison window once a pedestal has been established; then a delayed, scaled, and inverted signal is compared against the original, and the TOF event is marked when the Zero Crossing discriminator fires. Over the valid range of input signals, the TOF "walk" is less than $1 \mathrm{~ns}$. The minimum start-stop pulse pair resolution is $1.45 \mathrm{~ns}$. The longest valid TOF is $330 \mathrm{~ns}$. The maximum repetitive start rate is $8.3 \mathrm{MHz}$.

2.4.3.3 Charge Amplifier Board The Charge Amp Board (Fig. 26) uses thirteen A121 charge amplifiers to process the MCP Stop Events and determine the elevation angle of the measured particle. A zap-trap protected voltage follower amplifier process the charge input from each Stop Anode and converts it to a voltage waveform. That waveform is sent in two directions. It is sent to a summing amplifier and then on to the TOF board in order to represent the Stop Timing Pulse. Simultaneously, the voltage waveform is sent to the A121 for each Stop anode. Figure 26 shows a schematic of one channel. The A121 is set with a $3 \times 10^{6}$ electron threshold. The pulse width is set to $90 \mathrm{~ns}$ and the dead-time to $180 \mathrm{~ns}$. The output of the A121 is sent to the Digital Board, which is integrally connected to the Charge Amplifier Board through a common flexible circuit layer. Once on the Digital Board, the Stop anode signals are serialized and transmitted to the JSIB via two high-speed, LVDS serial transmission lines.

2.4.3.4 Digital Board The Digital Board is permanently connected to the Charge Amplifier Board through shared flex-circuit layers. The Digital Board registers the digital pulses from all thirteen of the A121 outputs and uses passive termination to translate the $5 \mathrm{~V}$ level outputs from the A121 to the $3.3 \mathrm{~V}$ serializer input levels. The serializer chip is employed to 
Fig. 26 JADE-I Charge

Amplifier Board

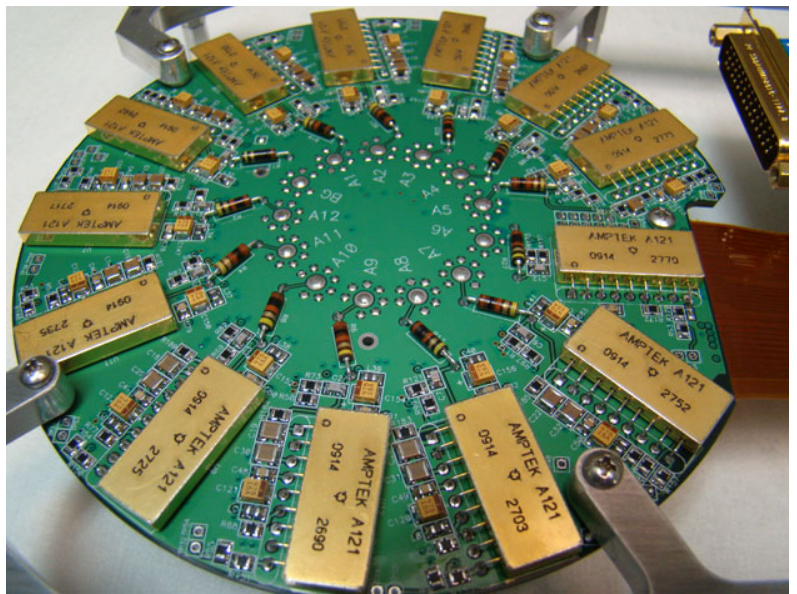

Fig. 27 JADE-I HVDB

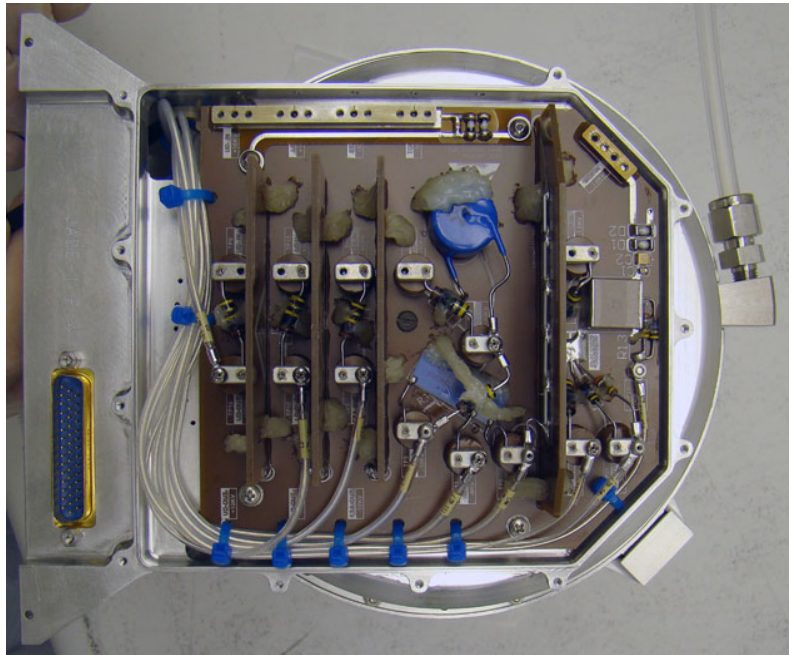

minimize wiring from the sensor electronics to the JADE Ebox; the signals are serialized into a frame and transmitted to the through high-speed LVDS lines to the Sensor Interface Board in the Ebox. The sampling clock frequency, the A121 pulse width, and the A121 dead-time were selected to ensure that at least one high-state and one low-state is transmitted serially for each of the inputs so that the JSIB can correctly count all pulses of all A121 channels at a periodic rate of $5.55 \mathrm{MHz}$ for each pixel. The serializer chip samples all of the inputs every $60 \mathrm{~ns}$, and then, using a phase-locked loop, transmits the samples down to the JSIB via two pairs of high speed LVDS serial lines. The LVDS signal levels allow high bandwidth transmission while maintaining the low EMI emissions required for compatibility with the MWR instrument.

2.4.3.5 JADE-I High Voltage Distribution Board The JADE-I HVDB (Fig. 27) provides shielded, mechanical termination of the doubly-shielded, high voltage coaxial cables; filters the detector bias signals; and provides dropping resistors to set the proper optical voltages in the detector. The distribution of voltages is shown in Fig. 28. 


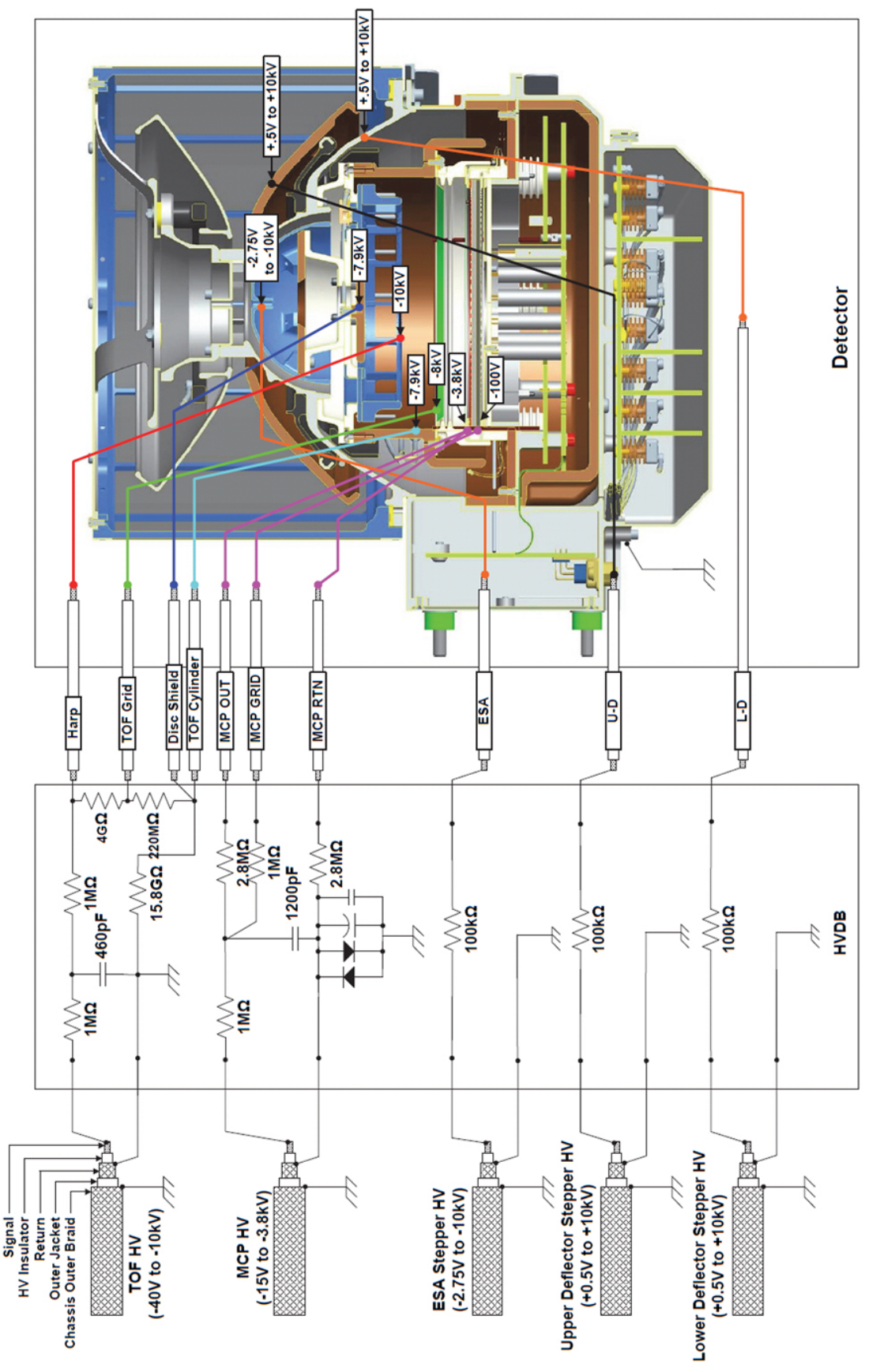

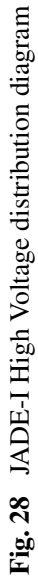




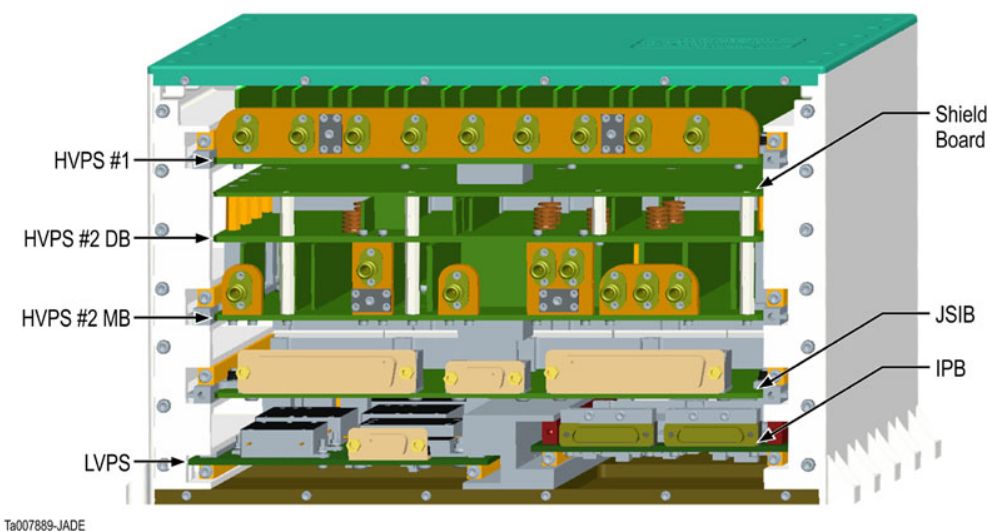

Fig. 29 The JADE Electronics Box

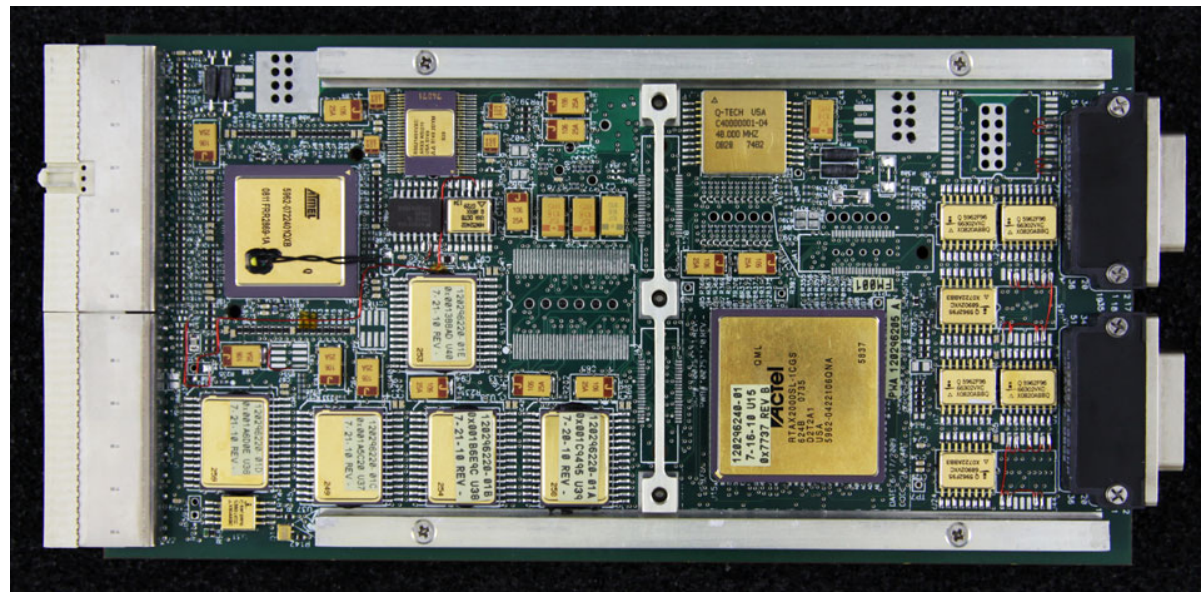

Fig. 30 Instrument Processor Board (IPB)

\subsubsection{JADE Electronics Box (Ebox)}

The JADE Ebox (Fig. 29) resides in the Juno Radiation Vault. No additional radiation shielding is required for the Ebox other than the titanium vault itself and the self-shielding provided by the other avionics in the vault. Within the Ebox are five Printed Wiring Modules and the Backplane. These modules include: The Low Voltage Power Supply (LVPS), the Instrument Processing Board (IPB), the JADE Sensor Interface Board (JSIB), and two High Voltage Power Supply modules (HVPS \#1 and HVPS \#2).

2.4.4.1 Instrument Processor Board The Instrument Processor Board (IPB, Fig. 30) is the computing platform on which the JADE Flight Software runs. Figure 31 shows a block diagram of the IPB. The IPB provides an Atmel AT697E SPARC8, RISC processor, capable of operating at 86 Dhrystone Million Instructions per Second (MIPS) or 23 Whetstone Million Floating Point Operations per Second (MFLOPS). The IPB also functions as a v2.2 PCI compliant cPCI bus master to communicate with the JSIB across the JADE backplane. 


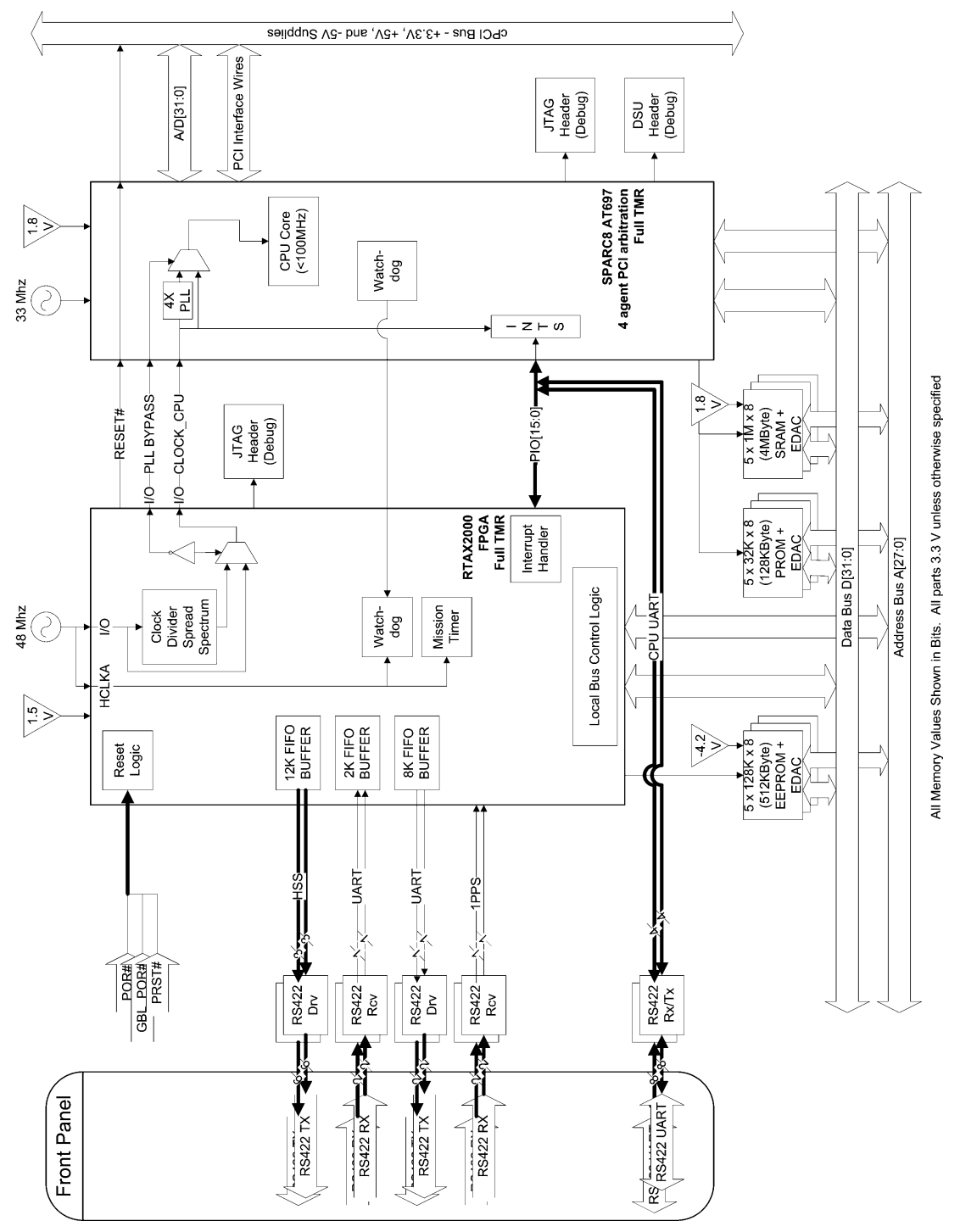

है 
Fig. 32 Low Voltage Power Supply Board

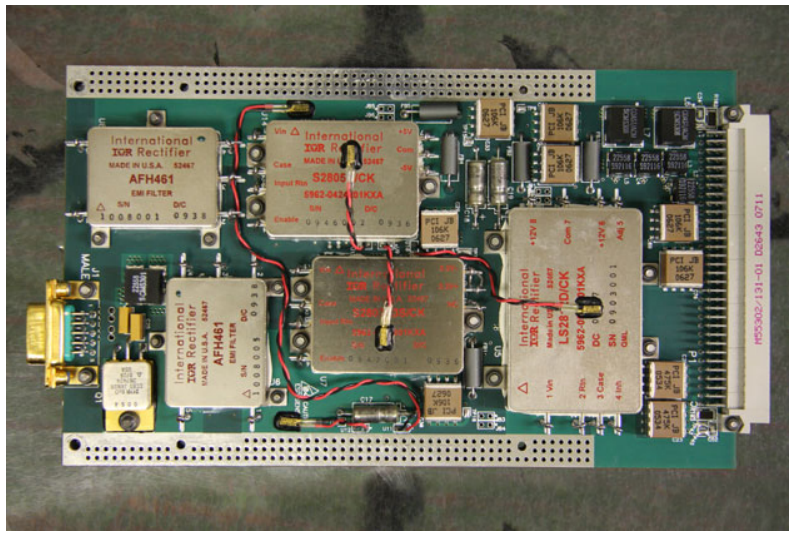

The IPB responds to spacecraft commands, transmits low-rate Science and Housekeeping telemetry, and transmits high-rate, synchronous Burst Mode Science data. Through the JSIB, the IPB controls the sensors and reads back telemetry data collected. The data is then formatted into packets and transmitted to the spacecraft.

The spacecraft synchronizes communication using the one half pulse-per-second (1/2PPS) signal. Every two seconds the spacecraft asserts a 1/2PPS signal, which is used in concert with a spacecraft command to precisely update JADE's $1 \mu$ s resolution system timer. Spacecraft commands are received and buffered on the IPB over a Universal Asynchronous Receive and Transmit (UART) interface. Low Speed Telemetry is written by the software into a buffer and transmitted to the Spacecraft over the UART interface. During Burst Mode, JADE transmits high speed burst data, which is written into a buffer and transmitted at a higher rate across a dedicated high-speed synchronous serial interface.

The IPB is responsible for system resets of JADE for operational and fault tolerant purposes. The JADE instrument can be reset by power cycling, spacecraft command, or JADE software reset. Watchdog resets for both the processor and the FPGA ensure a software fault does not cause an instrument failure.

The IPB has the following memory: $128 \mathrm{~KB}$ PROM for the boot code; $512 \mathrm{~KB}$ EEPROM to hold copies of the Flight Software and Flight Look-Up Tables; and 4 MB SRAM used for Code and Data when the software is executing. All memory uses a 7-bit Hamming Code for double bit error detection and single bit error correction, providing additional hardware fault tolerance.

The logic control of the IPB is contained within an ACTEL RTAX2000S-1 FPGA. All logic is Triple-Module-Redundant to decrease susceptibility to Single Event Upsets (SEUs). The FPGA drives the CPU clock and allows on-the-fly adjustment from $48 \mathrm{MHz}$ to $1 \mathrm{MHz}$. This allows the software to have access to substantial computing power for time-critical tasks, and then to throttle back to a very low processing speed to conserve overall power.

2.4.4.2 Low Voltage Power Supply The Low Voltage Power Supply (LVPS, Fig. 32) uses $28 \mathrm{~V}$ Spacecraft Primary power to generate $3.3 \mathrm{~V}, \pm 5 \mathrm{~V}$, and $\pm 12 \mathrm{~V}$ secondary power for the JADE instrument. JADE can be powered by redundant A- or B-side of the Juno Spacecraft inputs. These two power lines are diode OR-ed together. Inrush current is limited to reduce part stresses. EMI Filtering is performed to prevent spacecraft noise from affecting JADE and noise from JADE being reflected back to the spacecraft bus.

Separate sets of power rails provide different types of loads with their own sets of power planes in order to keep noisy loads from affecting quiet loads. JADE provides Digital Re- 


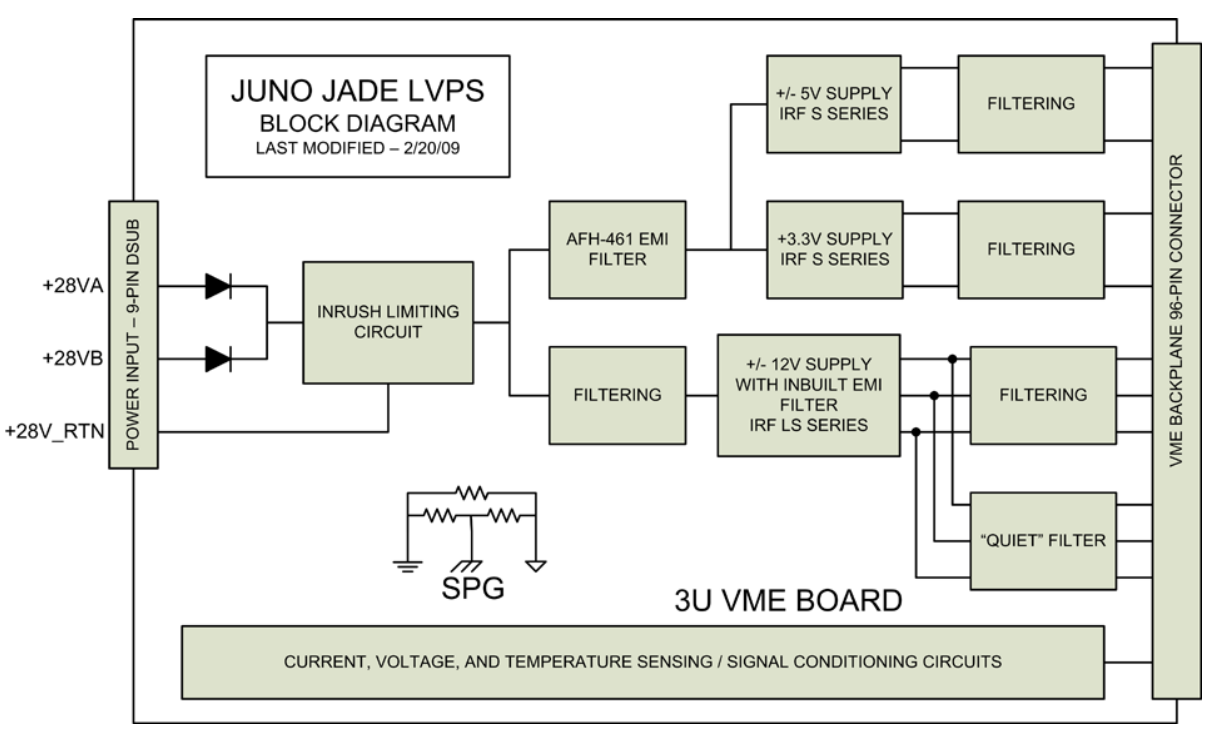

Fig. 33 LVPS block diagram

turns, Analog Returns, and High Voltage Returns. The returns are referenced to chassis through a resistor so that, under all test conditions, the power supply returns are chassis referenced. However, the Analog Grounds are tied to chassis at the sensors to maximize the noise performance of the Front End Analog Electronics in the sensors. The resistor that references ground to chassis prevents ground loops and keeps return currents on the paths provided in the wiring harnesses. Elements of the LVPS system are shown in Fig. 33.

2.4.4.3 JADE Sensor Interface Board The JADE Sensor Interface Board (JSIB) is the heart of the Ebox (see JSIB block diagram in Fig. 34). The JSIB commands all of the high voltage power supplies to their required levels, it opens an acquisition window, processes raw count data from all sensors, and determines coincidence in the JADE-I sensor while measuring the time-of-flight of the incoming particle. All of the control operations are governed by sweep tables that are loaded into memory by the IPB. Once the JSIB is enabled it begins processing events from the sensors and builds up double-buffered histograms that are turned into science telemetry products by the IPB.

The JSIB contains a high speed Digital-to-Analog Converter to generate the specific voltages required by the sweep tables. Once the level is generated it is captured by one of many Sample-and-Hold Amplifiers which directly drive the analog command level, across the backplane, to each of the HVPS inputs.

Raw anode counts from each of the sensors are de-serialized by the JSIB and recorded in the appropriate histogram or real-time count-rate register. The JSIB measures the JADE-I TOF by using a Time-to-Digital Converter (TDC) that consists of a coarse counter that is generated off of the $62.5 \mathrm{MHz}$ clock and a circuit that also captures, using delay lines, the position of the start and stop pulses with a resolution approximately ten times finer than the 62.5 MHz clock pulses themselves (Fig. 35). Coincidence logic determines what ion event has occurred. Using the measured TOF as well as the commanded ESA and DFL settings, the event is classified by ion species and is recorded in histograms using table-driven logic. 


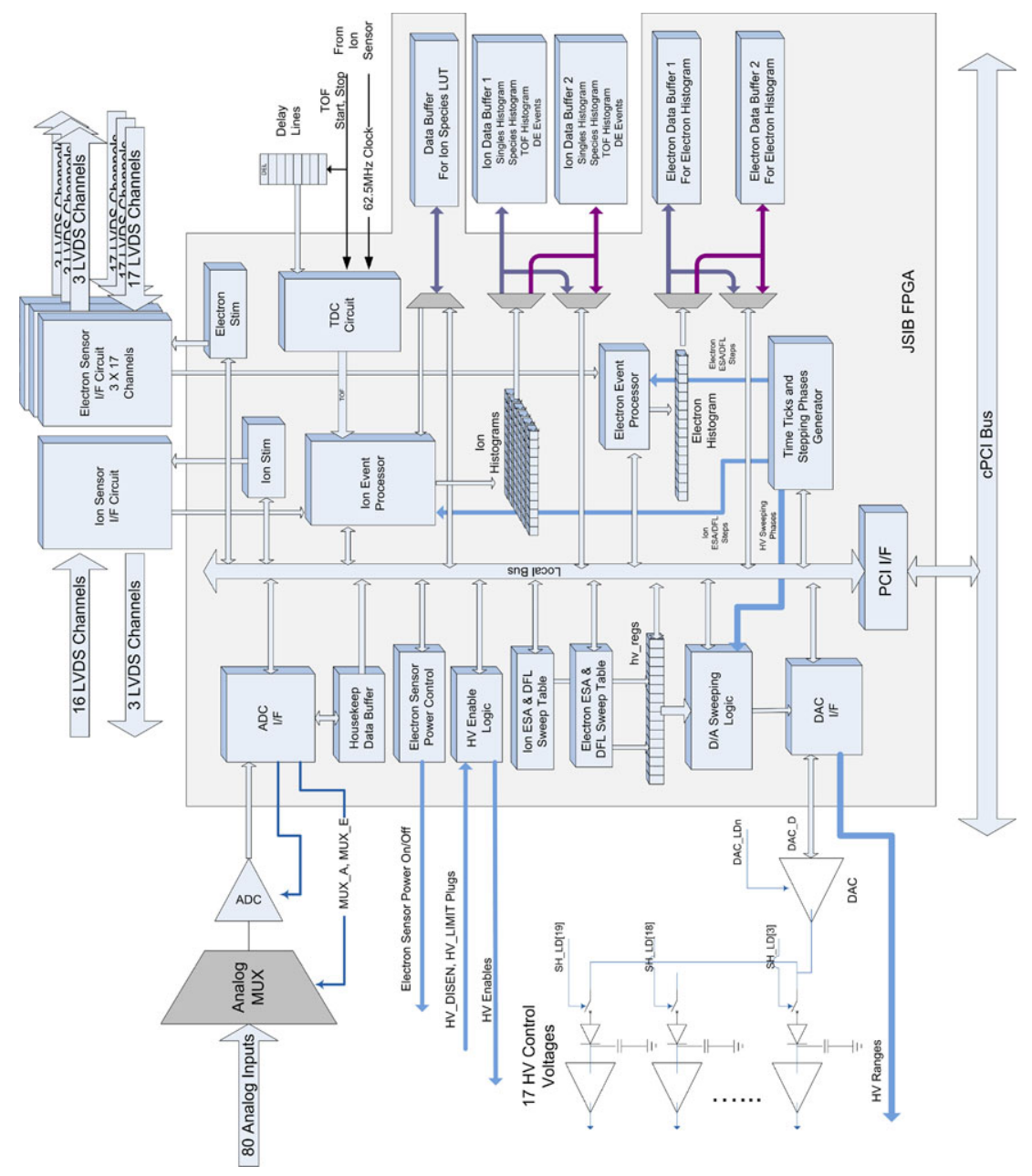

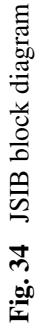


Fig. 35 JSIB TOF timing diagram

Fig. 36 JADE Sensor Interface Board photograph

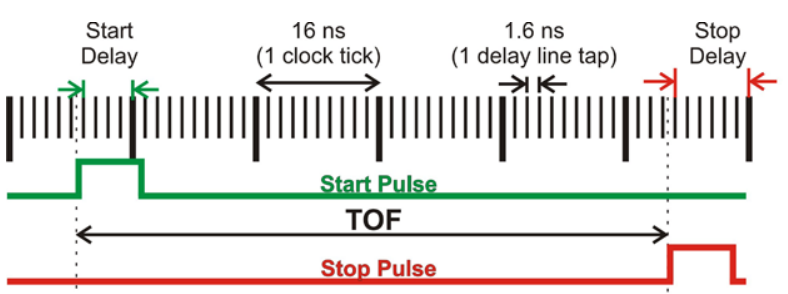

TOF $=$ number of clock ticks between start and stop pulse + Start Delay - Stop Delay $=5 \times(16 \mathrm{~ns})+5 \times(1.6 \mathrm{~ns})-7 \times(1.6 \mathrm{~ns})=76.8 \mathrm{~ns}$ (for the above example)

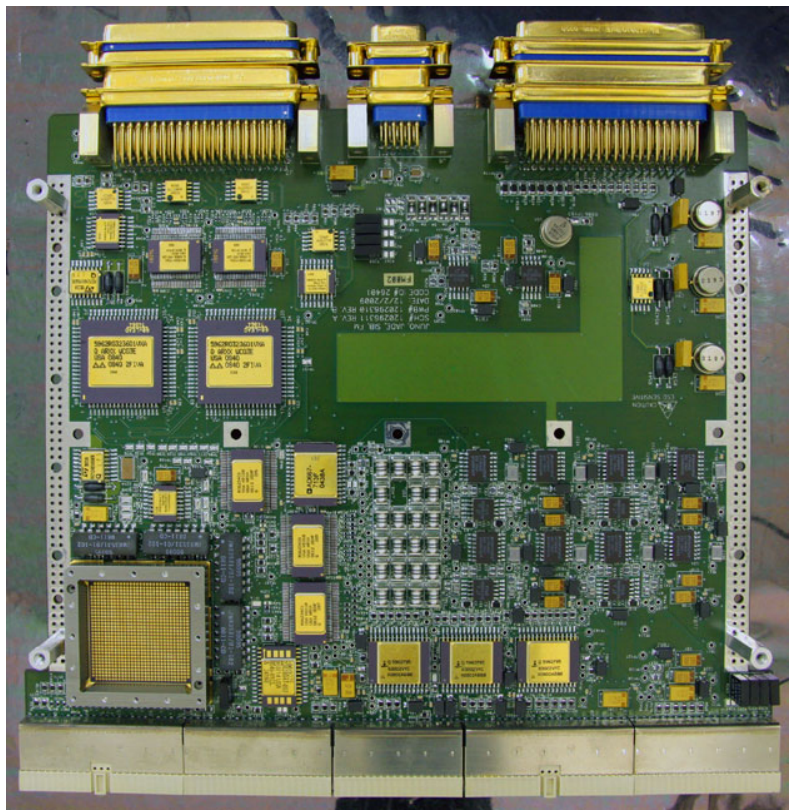

Test pulsers to all of the sensors can be generated by the JSIB so that the amplifiers in each of the sensors can be checked at any time. Various rates and combinations can be selected. In the case of JADE-I various TOFs are also selectable.

The JSIB measures 80 different housekeeping analog values and stores them in a buffer that can be read out by the IPB and sent to the ground to monitor the health and safety of the JADE instrument. The JSIB communicates over PCI backplane to the IPB. The JSIB is a $6 \mathrm{U}$ board (Fig. 36) and uses the spare connector pins to communicate analog commands, analog monitors, and various enable and safe signals to the HVPS over the backplane.

2.4.4.4 High Voltage Power Supply This HVPS provides all of the power to the MicroChannel Plate (MCP) detectors and sensor electro-optics. Although the two HVPS boards are host to 17 different power supplies, each sensor's power supplies are kept independent from one another to maximize fault tolerance. For each JADE-E sensors there is a programmable MCP supply as well as three stepping supplies: DFL-UP, DFL-DN, and ESA. For JADE-I there are programmable MCP and TOF supplies as well as three stepping supplies: DFL-UP, DFL-DN, and ESA. Table 7 defines the key design requirements for the HVPS. 
Table 7 HVPS key design requirements

\begin{tabular}{lll}
\hline Circuit & Parameter & Specification \\
\hline HVPS & Input voltage & $\pm 12 \mathrm{~V} \pm 5 \%$ \\
BULK & Enable/Disable & $+12 \mathrm{~V}$ open collector input, Hi $=$ Enable \\
BULK & Voltage monitor & 0 to $4.5 \mathrm{~V}=0$ to $10.5 \mathrm{KV}$ \\
BULK & V/10 & Input low $=(\div 10)$ \\
STEPPER & Range select & $+12 \mathrm{~V}$ open collector input, Low $=$ Low range \\
STEPPER & High range output accuracy & 0 to $-4.5 \mathrm{~V}$ input $=0$ to $10 \mathrm{KV} \pm 1 \%$ of full scale output \\
STEPPER & Monitor accuracy & 0 to $10 \mathrm{KV}=0$ to $4.5 \mathrm{~V} \pm 2 \%$ \\
STEPPER & Low range output accuracy & 0 to $-4.5 \mathrm{~V}$ input $=0$ to $316 \mathrm{~V} \pm 1 \%$ of full scale output \\
STEPPER & Monitor accuracy & 0 to $316 \mathrm{~V}=0$ to $4.5 \mathrm{~V} \pm 2 \%$ \\
STEPPER & Minimum slew rate & $520 \mathrm{~V} / \mathrm{mS}$ with load $=430 \mathrm{pF}$ \\
MCP & Enable/Disable & $+12 \mathrm{~V}$ open collector input, Hi $=$ Enable \\
MCP & Output & 0 to $-4.5 \mathrm{~V}$ input $=0$ to $3.8 \mathrm{KV} \pm 1 \%$ of full scale output \\
MCP & Voltage monitor & 0 to $3.8 \mathrm{KV}=0$ to $4.5 \mathrm{~V} \pm 2 \%$ \\
MCP & Current monitor & 0 to $120 \mathrm{uA}=0$ to $4.5 \mathrm{~V} \pm 2 \%$ \\
TOF & Enable/Disable & $+12 \mathrm{~V}$ open collector input, Hi $=$ Enable \\
TOF & Output & 0 to $-4.5 \mathrm{~V}$ input $=0$ to $10 \mathrm{KV} \pm 1 \%$ of full scale output \\
TOF & Voltage monitor & 0 to $10 \mathrm{KV}=0$ to $4.5 \mathrm{~V} \pm 2 \%$ \\
\hline
\end{tabular}

On the HVPS Board 1, there is a $+10.5 \mathrm{kV}$ bulk power supply for each JADE-E sensor (see HVPS Board 1 block diagram in Fig. 37). Three linear regulator supplies use this bulk supply to produce the $+10 \mathrm{~V}$ to $+10 \mathrm{kV}$ stepping supplies for the DFL-UP, DFL-DN, and ESA optical voltages which set the acceptance angle and the acceptance energy of each sensor. Each of these supplies can be commanded to be in low-range or high-range mode. If they are in high range, then the analog command level is interpreted to control the stepping supply across the full $+10 \mathrm{kV}$ range. If they are in low-range, the analog command level is interpreted to control the stepping supply across the range $10 \mathrm{~V}$ to $316 \mathrm{~V}$.

On HVPS Board 2 there are four programmable MCP power supplies (see HVPS Board 2 block diagram in Fig. 38). Three $+3.8 \mathrm{kV}$ supplies are for the JADE-E MCPs. The remaining supplies on Board 2 are used for the JADE-I sensor. One $-3.8 \mathrm{kV}$ supply is for the JADE-I MCP. There is also one $-10 \mathrm{kV}$ programmable TOF power supply. To supply the DFL-UP and DFL-DN voltages, there is a $+10.5 \mathrm{kV}$ bulk power supply that supplies the voltage for the two deflector linearly regulated stepping power supplies. To supply the ESA voltage, there is a $-10.5 \mathrm{kV}$ bulk power supply that supplies the voltage for the ESA linearly regulated stepping power supply.

The power supplies were designed to be disabled, limited, or armed by means of a Safe/Arm Plug on the Ebox. No voltage is generated when the HVPS is disabled. When the HVPS is limited the supply cannot be commanded to full voltage and instead is limited to non-vacuum safe levels. When no plug is installed, as is the case for flight, then the HVPS supply is armed and software can enable and control the HPVS to full voltage levels.

2.4.4.5 Backplane The backplane provides the interconnections between all of the boards in the Ebox. All of the connectors are custom pinouts on standard 96-pin VME connectors with the exception of the connection between the IPB and the JSIB, which are interconnected using a standard PCI interface. This provided the high speed communication path between 


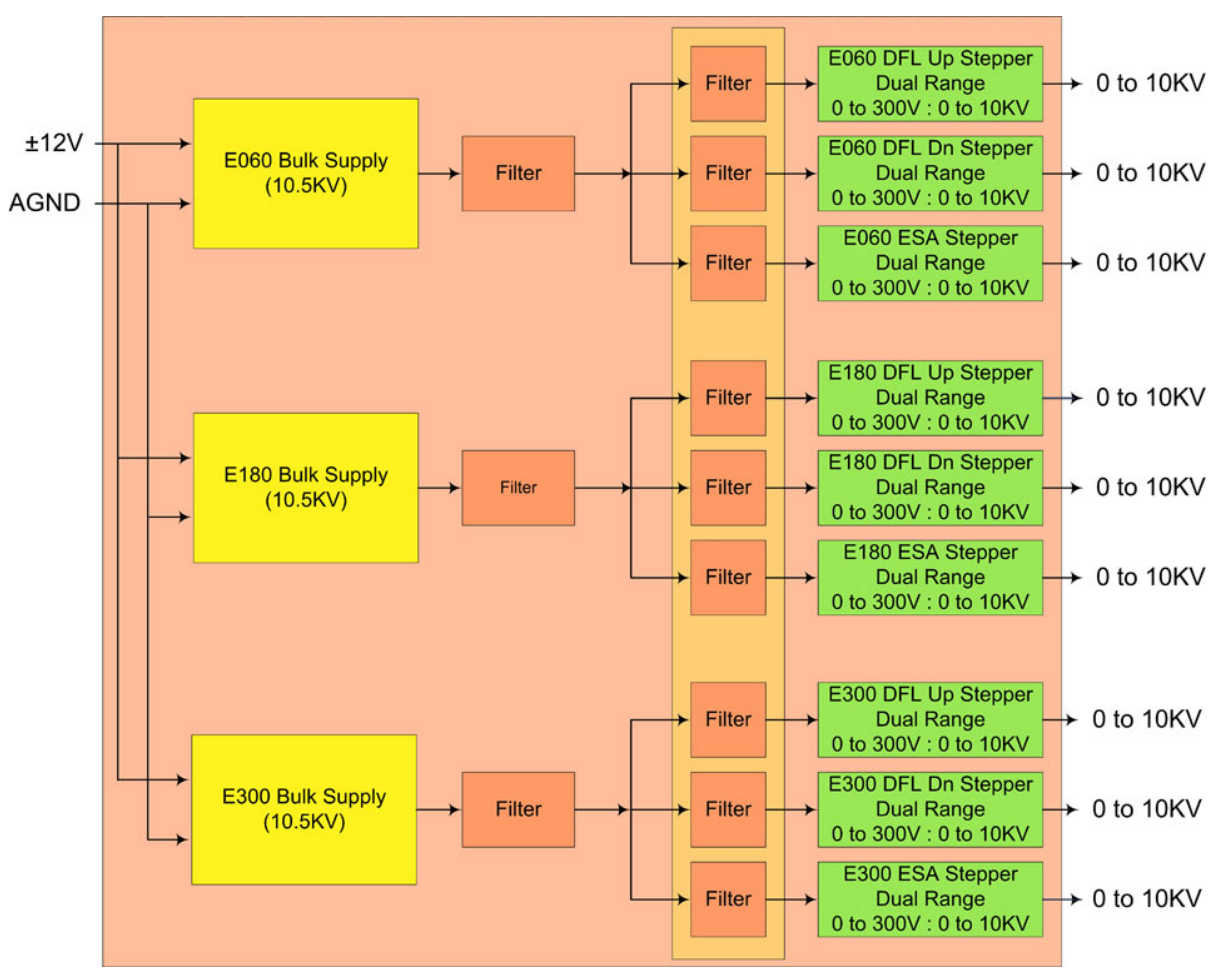

Fig. 37 HVPS Board 1 block diagram

the processor board and the sensor interface board. The ground planes in the backplane are systematically segmented to provide well-separated, low-impedance power and ground planes for the each of the types of signal and load: digital, analog, and high voltage. This maintains the signal integrity of each subsystems and helps to minimize noise and cross-talk.

2.4.4.6 Intra-instrument Harness All of the signals for the four sensors are contained in an intra-instrument harness that connects them to the Ebox in the titanium radiation vault. There is a custom radiation vault plate that these signals pass through and the low voltage signals have standard, bulkhead D-connectors in this plate. To avoid the addition of any high voltage connectors, all sensor high voltage cables are pigtailed from the HVPS and pass through mouse-holes in the radiation vault plate.

\subsubsection{JADE Resources}

A summary of JADE resources is provided in Table 8.

\subsection{Radiation Shielding Calculation and Modeling}

The radiation shielding requirements ensure that the background counting rates for the four (3-E and 1-I) JADE sensors are sufficiently low to allow an adequate signal-to-noise ratio (SNR) in the auroral regions for all orbits, and enable JADE to satisfy its science requirements. 


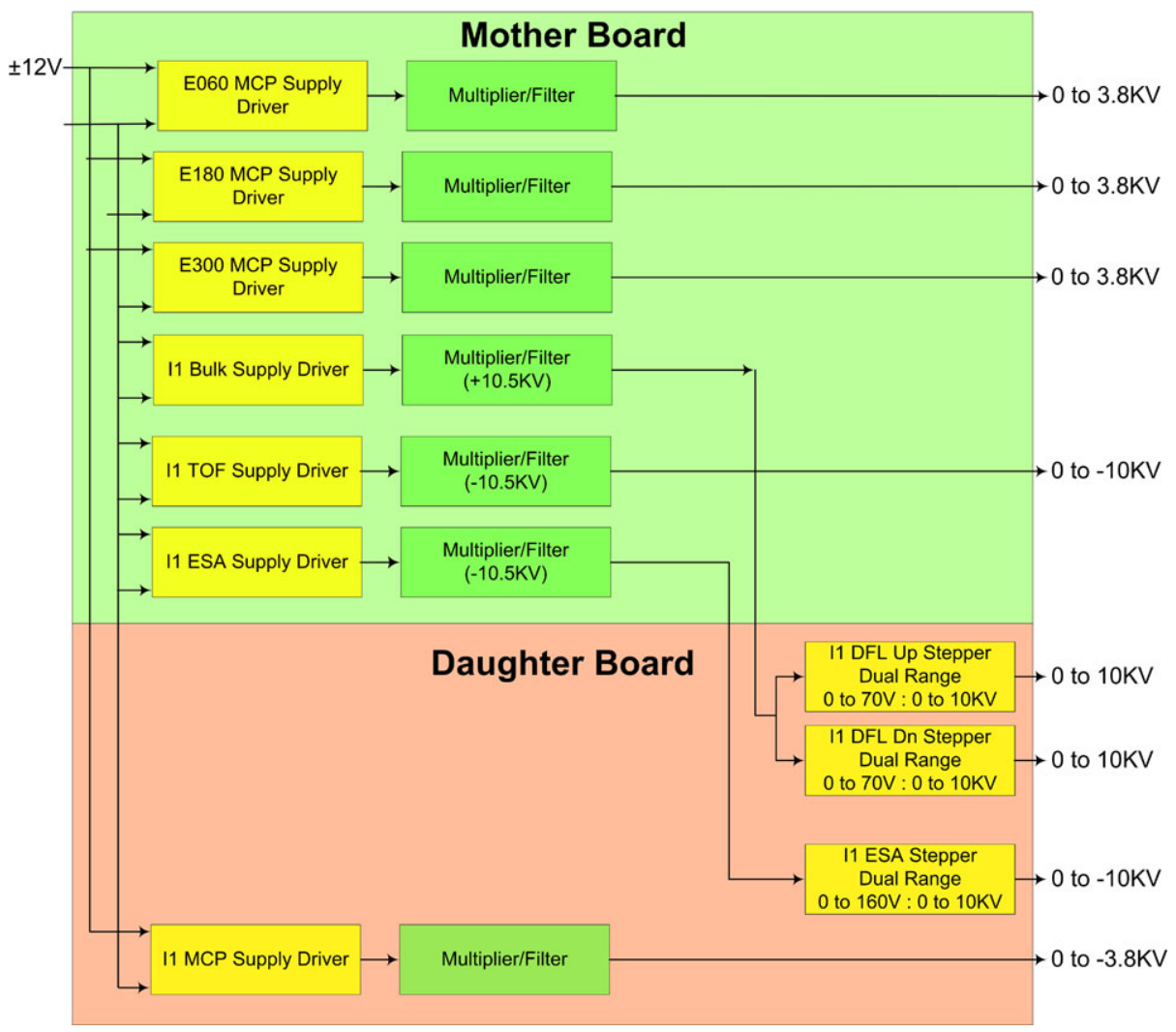

Fig. 38 HVPS Board 2 block diagram

For JADE-E it was not possible to remove background by using a coincidence measurement. Thus, the shielding must attenuate the penetrating radiation adequately so that its signal is lower than the Jovian electron signal. For JADE-E we defined a threshold for a minimum signal of 10 counts per energy step, which produces a $30 \%$ Poisson uncertainty. The energy step duration is $1 / 64 \mathrm{~s}$ minus $2 \mathrm{~ms}$ of settling time, resulting in $\sim 13.6 \mathrm{~ms}$, and the lowest rate is $10 / 13.6=\sim 0.734 \mathrm{kHz}$ per anode pixel $\left(\sim 2 \mathrm{kHz} / \mathrm{cm}^{2}\right)$. Therefore, at a minimum the shielding must prevent the background from exceeding $\sim 2 \mathrm{kHz} / \mathrm{cm}^{2}$ for an SNR of 1.

The JADE-I geometric factor is designed to yield a peak rate of $200 \mathrm{kHz}$. Based on observations of the terrestrial aurora, we assume that features with important physical properties can have fluxes down to $\sim 1 \%$ of the peak. Therefore, a weak but important signal level is $\sim 2 \mathrm{kHz}$, and the SNR should be high enough to yield a good measurement.

\subsubsection{Jupiter's Environment}

The primary sources of high-energy, charged-particle radiation for the Juno mission are the Jovian electrons and protons in the trapped radiation belts. In addition, there is some smaller contribution from solar protons and galactic cosmic rays. For Juno's orbit, sources internal to Jupiter's magnetosphere will certainly provide the most significant contribution to the instantaneous background counting rates in JADE as well as in JEDI and the various imagers. 
Table 8 JADE resource summary table

\begin{tabular}{|c|c|c|c|}
\hline Item & Mass [grams] & Power $[\mathrm{mW}]$ & $\begin{array}{l}\text { Overall dimensions } \\
(\mathrm{wd} \times \mathrm{dp} \times \mathrm{ht})[\mathrm{cm}]\end{array}$ \\
\hline JADE-E sensor-total & 5240 & 1000 & $21 \times 21 \times 21$ \\
\hline Electro-optics & 891 & & \\
\hline Radiation shielding & 2700 & & \\
\hline Anode board + MCP & 123 & 0 & $14 \times 7 \times 1$ \\
\hline Capacitor board & 69 & 0 & $15 \times 7 \times 3$ \\
\hline Charge amplifier board & 53 & 700 & $15 \times 7 \times 3$ \\
\hline Digital board & 69 & 100 & $16 \times 7 \times 2$ \\
\hline High voltage distribution board & 103 & 200 & $12 \times 10 \times 3$ \\
\hline Housing & 1232 & & \\
\hline JADE-I sensor-total & 7552 & 1900 & $18 \times 24 \times 22$ \\
\hline Electro-optics & 2526 & & \\
\hline Radiation shielding & 4540 & & \\
\hline Anode board & 44 & & $7 \times 7 \times 1$ \\
\hline Time-of-flight board & 57 & 1700 & $7 \times 7 \times 2$ \\
\hline Charge amplifier board & 141 & & $7 \times 7 \times 2$ \\
\hline Digital board & 48 & & $9 \times 6 \times 2$ \\
\hline High voltage distribution board & 116 & 200 & $12 \times 12 \times 3$ \\
\hline Housing & 576 & & \\
\hline JADE Ebox-total & 15200 & & $27 \times 23 \times 17$ \\
\hline Instrument processor board & 450 & 1500 & $12 \times 21 \times 3$ \\
\hline Low voltage power supply & 552 & 4200 & $12 \times 21 \times 3$ \\
\hline JADE sensor interface board & 560 & 3300 & $22 \times 21 \times 3$ \\
\hline High voltage power supplies & 3156 & 9100 & $22 \times 21 \times 8$ \\
\hline Backplane & 338 & 0 & $20 \times 15 \times 3$ \\
\hline Housing & 4314 & & $27 \times 23 \times 17$ \\
\hline Intra-instrument harness and vault plate & 5830 & & \\
\hline JADE grand total & 38500 & 21000 & \\
\hline
\end{tabular}

Of the trapped Jovian particles, most of the relevant radiation comes from electrons, which thus provide the dominant contribution to the average instantaneous background counting rate, integral fluences, and peak fluxes during the Juno mission. Therefore, in this section we focus on these dominant effects from the Jovian electrons. Even though prior missions to Jupiter such as Pioneer 10 and 11, Voyager 1 and 2, Ulysses, and Galileo have recorded the distribution of electron and proton fluxes at various (mostly equatorial) locations in its magnetosphere, they did not provide the necessary measurements to specify the radiation environment for the polar-orbiting Juno mission.

In order to assess the radiation environment along Juno's orbit, we used two different, but complimentary, models: the JPL Divine-Garrett (JPL-DG) empirical model and the SwRI Santos-Costa (SwRI-DSC) physics-based model. Both radiation models require trajectory information in the form of spacecraft range, latitude, and west longitude at fixed time-interval steps. The SOAP (Satellite Orbit Analysis Program) software program (Santos-Costa and Bourdarie 2001) generated position information at 60-s intervals, us- 
Fig. 39 Juno's trajectory for orbits at the start (horizontal), middle, and end (highly inclined) of the nominal science mission. The yellow-shaded portions roughly indicate periods when Juno is in the auroral region (see text for details)

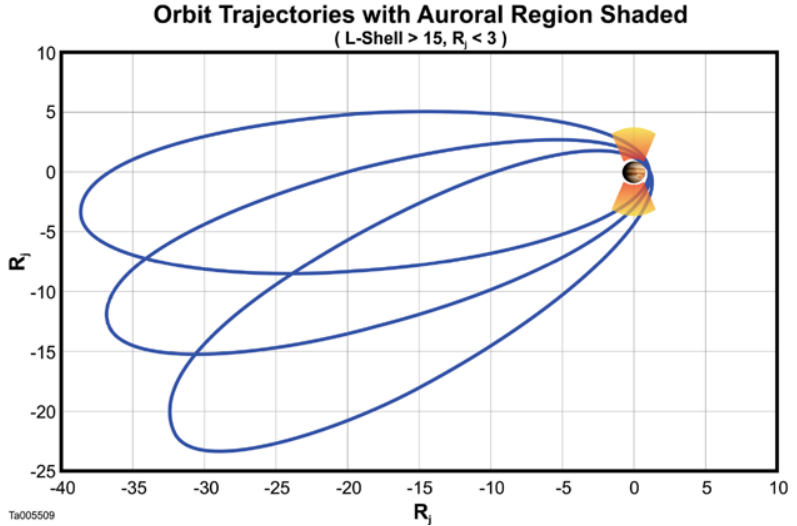

ing the spacecraft ephemeris file Juno-110818EDP-120918DSM-161022AD-4018kmPJ0to-pj33.bsp. The location of Juno for the start, middle, and end of the primary mission are shown in Fig. 39.

In this figure we have yellow-shaded portions of the orbits above the poles to guide the eye toward periods when Juno takes measurements in the Jovian auroral region. The main aurora occurs on $L$-shells greater than 15 . Ultraviolet images show an auroral spot on field lines connected to Ganymede, which is at $L=15$ and consistently equatorward of the main oval (Clarke et al. 2002). The altitude of the auroral acceleration region is uncertain, and existing estimates focus on the Io auroral spot. However, both theoretical models (e.g., Su et al. 2003) and the size of a source cone observed in energetic electrons (Williams et al. 1999) suggest that the acceleration region is between 1.5 and $2 R_{J}$ from body center. By focusing on the region inside $3 R_{J}$ we allowed for the inherent uncertainties in these results. Owing to these considerations, we defined the "auroral region" here as portions when the spacecraft range $R<3 R_{J}$ and $L$-shell $L>15$.

The two models also required a Jovian magnetic field model to define $L$-shells. The inner and outer radiation models were developed by Divine and Garrett (McAlpine 2007) and refined using actual spacecraft measurements when available. The refined model was then used to simulate the electron fluxes at various energies along Juno's orbit.

We use both the JPL-DG and SwRI-DSC models to estimate the differential and integral electron fluxes at various portions of Juno's orbit. The SWRI-DSC model was developed using adiabatic invariants in phase space (Northrop 1963), and solves the Fokker-Planck equation (Schulz and Lanzerotti 1974) for determining the time evolution of Jovian radiation-belt electron-distribution functions. This model includes processes affecting off-equator particles by employing a diffusion theory (Roederer 1970). Therefore, the calculation of the radiation belt's steady state was neither constrained by in situ data in the entire inner magnetosphere nor by empirical pitch-angle distributions for fitting radio measurements. The steady state was computed from inward particle transport and balancing losses and sources.

The SwRI-DSC model also includes sources related to electron populations observed in Dec. 1995 by the Galileo spacecraft just inside Io's orbit and losses associated with dust, satellites, Coulomb collisions, synchrotron radiation, and radial diffusion (SantosCosta 2001; Santos-Costa and Bourdarie 2001). This physics-based model was validated by performing comparisons with in situ and remote measurements (Santos-Costa 2001; Santos-Costa and Bolton 2008).

Merging with the original Divine and Garrett model (Divine and Garrett 1983) was performed at $L$-shells close to Io. A Fortran subroutine was written for computing coefficients, 


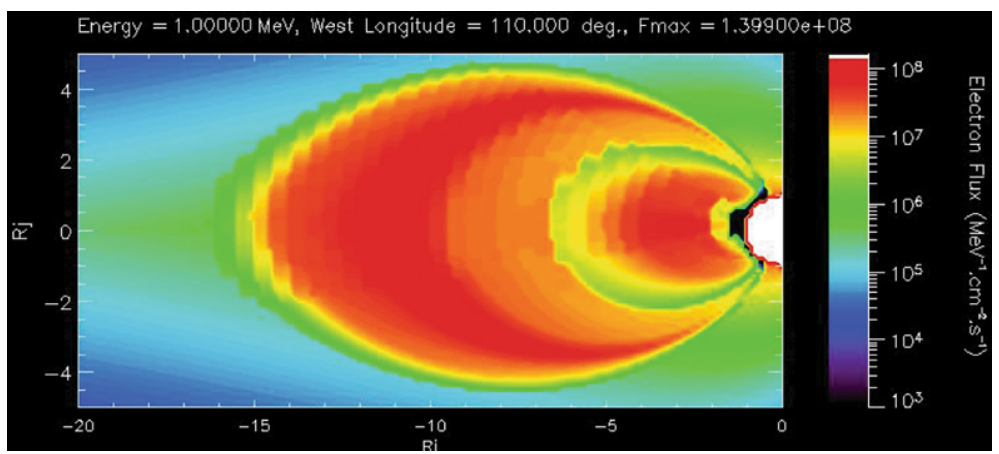

Fig. 40 Differential fluxes from the SwRI-DSC physical model for $L<6$ and the JPL-DG model for $L>6$. The differences between the physical model and JPL code for $L<6$ are significant only in relation to the differential fluxes; the simulation results from the two models are similar in terms of the integral flux

allowing a smooth transition between the differential fluxes provided by the SwRI-DSC $(L<6)$ and JPL-DG models. The coefficients were energy, latitude, and radial dependence at an $L$-shell of $\sim 6$. The merging method does not change the fluxes returned by the models outside this transitional region. Figure 40 shows simulation results obtained by merging the models.

We use the above models, which include much higher energy particles, solely to determine the amount of shielding required to make the JADE measurements and do not intend these results as predictions for actual fluxes to be observed in the JADE energy range. First, we extracted the maximum auroral integral flux above a given electron energy from the simulated fluxes from the merged JPL-DG and SwRI-DSC models at every spacecraft position for all 32 orbits. The radiation model provides a time-averaged value of the flux as a function of position. Temporal deviations of the flux from its average value have not been well-characterized and are not represented in the models. Figure 41 shows the peak integral fluxes in the auroral region for all orbits. The difference between the expected fluxes in the auroral region for various orbits is approximately half an order of magnitude.

\subsubsection{Radiation Shielding}

The shielding thickness can be calculated to stop electrons above a given threshold. However, in the process of stopping or slowing down these electrons, secondary radiation (e.g., electrons, Bremstrahlung radiation in the form of X-rays, and $\gamma$-rays) is generated that may provide an additional source of background in the detectors. Using the Geant 4 software (open source software downloadable from http://geant4.web.cern.ch/geant4/), we simulated the transmission of primary and generation of secondary particles resulting from interactions between a tantalum shield and $>1 \mathrm{MeV}$ electrons as they either penetrate or get stopped. The results were (1) that the background due to primary electrons was greatly attenuated by the Ta shield - the thicker the shield, the greater the attenuation, and (2) that the background count rates from secondary $\gamma$-rays are more than 10 times the rates from primary and secondary electrons. Also, the shielding thickness appears to only slightly affect the production of the $\gamma$-ray flux and background count rates.

A composite material helps reduce the production of secondaries. For example, a low atomic number (Z) material such as aluminum produces fewer $\gamma$-rays (low Brehmstrahlung production rate) but still decelerates electrons because it has a relatively high stopping power 


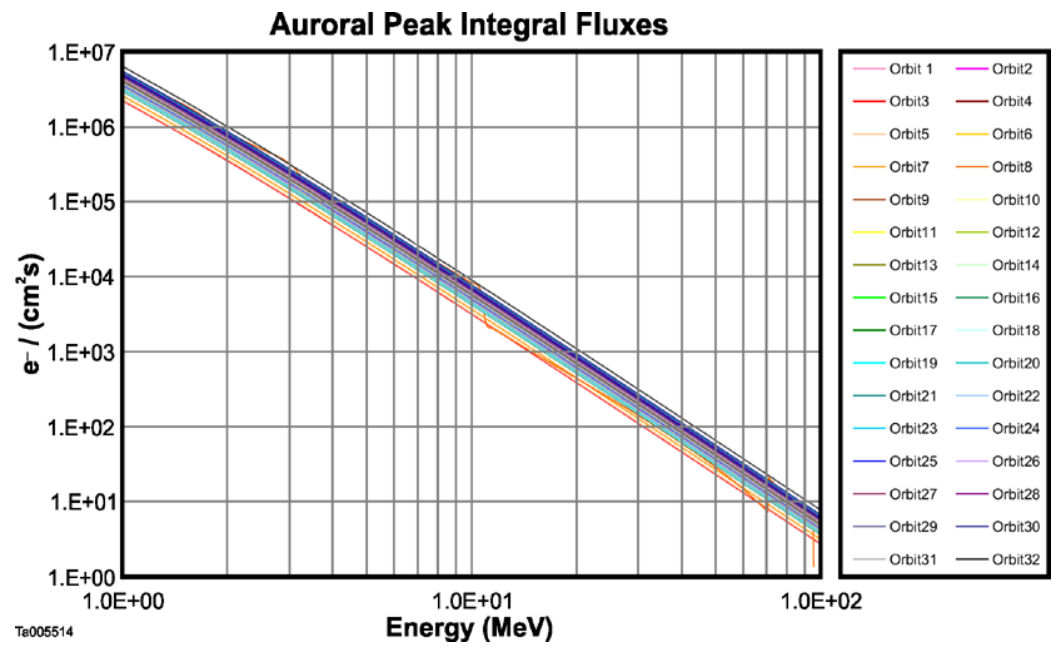

Fig. 41 Peak integral fluxes from the auroral region for all orbits (note that orbit numbering here starts with the first complete 11-day orbit). Since the auroral region is in $L$-shells $>6$, the JPL-DG model was used to calculate integral fluxes. The SwRI-DSC model was applied only to orbit segments where $L<6$
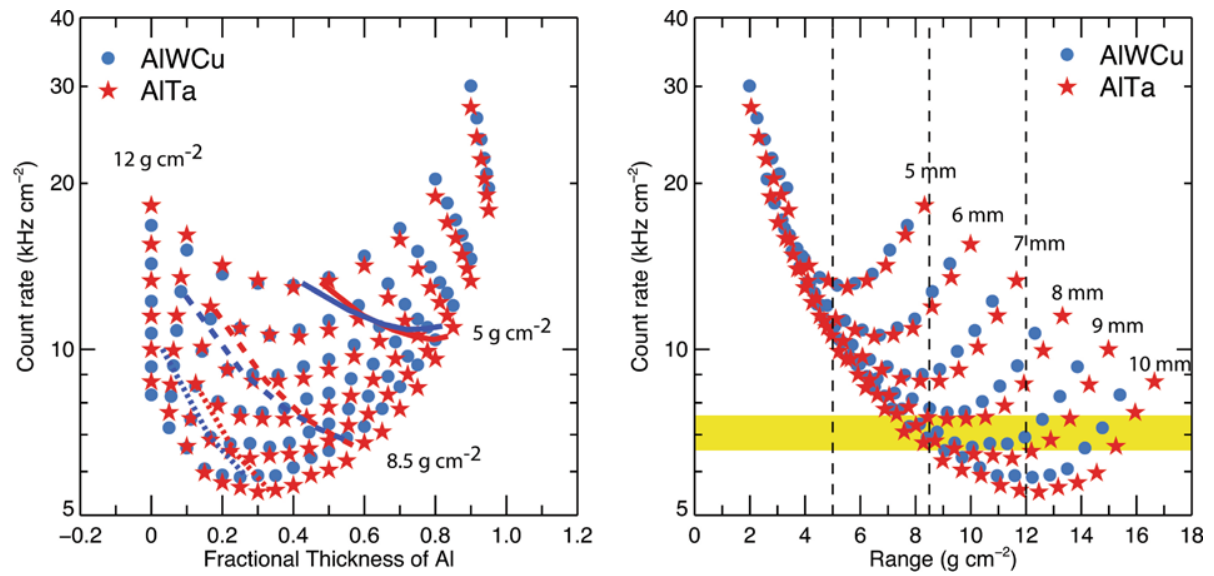

Fig. 42 Estimated MCP count rate plotted vs. (a) the fractional thickness of Al, and (b) the stopping power of the graded- $Z$ shield

per unit mass for energetic electrons. A higher $\mathrm{Z}$ material such as tantalum then attenuates the low-energy $\gamma$-rays from the $\mathrm{Al}$ and produce less energetic $\gamma$-rays because the electrons are decelerated. We chose to use a graded- $\mathrm{Z}$ combination of $\mathrm{Al}$ and $\mathrm{Cu} / \mathrm{W}$ alloy for JADE-I, and $\mathrm{Al}$ and Ta for JADE-E.

In order to optimize the fractional thicknesses of a graded low-Z/high-Z shield, we ran numerous simulations with these two material combinations with total thicknesses of 5, 6, 7, $8,9,10 \mathrm{~mm}$. In each successive run, we varied the Al thickness in increments of $0.5 \mathrm{~mm}$. For each simulation, we estimated the peak MCP rate due to transmitted electrons and photons. We assumed an MCP detection efficiency of $30 \%$ for high energy electrons and $11 \%$ for $\mathrm{X}$-rays and gamma rays (H. Funsten et al., personal communication, 2008). Figure 42 shows 


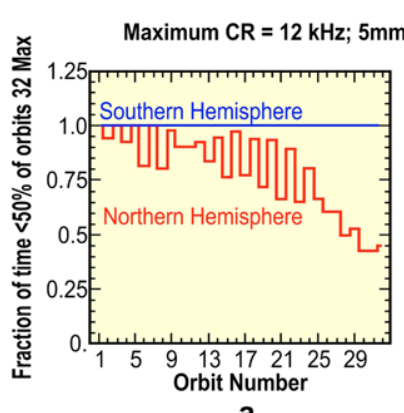

Ta007816-NADE

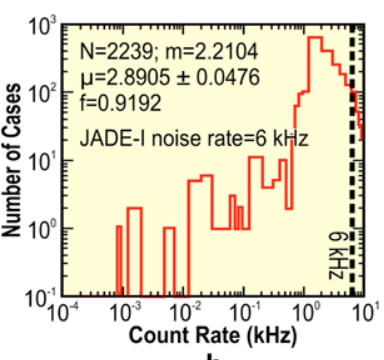

b

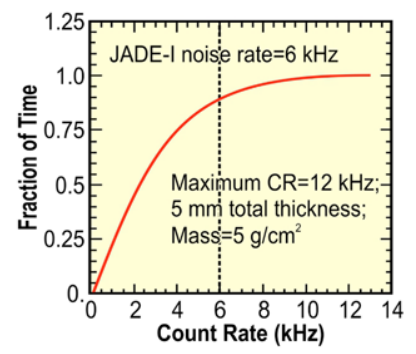

C

Fig. 43 For JADE-I: (a) fraction of time that background count rate are below $6 \mathrm{kHz}$ for each of Juno's orbits in the northern and southern hemispheres; (b) number of cases vs. estimated background count rate for JADE-I; and (c) integral fraction of time above various count rates

the estimated MCP count rate plotted versus (a) the fractional thickness of Al, and (b) the stopping power of the shield. In the left panel, the sets of curves represent stopping powers of $5,8.5$, and $12 \mathrm{~g} / \mathrm{cm}^{2}$. The left panel clearly shows that for a given total shield thickness, the minimum count rate is achieved when the fractional thickness of $\mathrm{Al}$ is 30 to $40 \%$. Vertical dashed lines are shown in the right panel at ranges of $5,8.5$, and $12 \mathrm{~g} / \mathrm{cm}^{2}$.

The yellow band in Fig. 42 shows the required stopping power for keeping the background count rate at or below $7 \mathrm{kHz} / \mathrm{cm}^{2}$. Since these rates are normalized to the peak auroral flux of $\sim 2 \times 10^{6}$ at $1 \mathrm{MeV}$ during orbit 32 , the figure shows that the background count rate can be kept below $\sim 7 \mathrm{kHz}$ during most of the prime mission for a range of total thicknesses and stopping powers.

The mass available for shielding corresponds to $\sim 8.5 \mathrm{~g} / \mathrm{cm}^{2}$ for JADE-E and $5 \mathrm{~g} / \mathrm{cm}^{2}$ for JADE-I, which prevents the maximum background rate from being lowered to $2 \mathrm{kHz} / \mathrm{cm}^{2}$ for JADE-E. However, Sect. 2.5.3 explains that the background rate will be less than $7 \mathrm{kHz}$ most of the time in the auroral regions.

\subsubsection{Background Estimates}

2.5.3.1 JADE-I Using the model electron fluxes throughout the auroral regions (e.g., from Fig. 41), we can estimate the fraction of time that the JADE-I background count rate is below $6 \mathrm{kHz}$ for each orbit using a graded-Z shield with a stopping power of $5 \mathrm{~g} / \mathrm{cm}^{2}$ and $5 \mathrm{~mm}$ total thickness. Note that the peak background rate due to transmitted photons and electrons through a $5 \mathrm{~g} / \mathrm{cm}^{2}$ shield, corresponding to the peak auroral flux expected during orbit 32 , is $\sim 12 \mathrm{kHz}$ (Fig. 42).

For a shield thickness of $5 \mathrm{~g} / \mathrm{cm}^{2}$, Fig. 43 shows that (1) the JADE-I count rate will remain below $\sim 6 \mathrm{kHz}$ throughout the southern hemisphere passes, (2) the fraction of time that the JADE-I background count rate will exceed $6 \mathrm{kHz}$ increases with increasing orbit number, (3) the mean $(\mu$ ) and median $(\mathrm{m})$ background rate for JADE-I is $\sim 2.9$ and $2.2 \mathrm{kHz}$, respectively, and (4) the JADE-I background count rate will remain below $\sim 6 \mathrm{kHz}$ for $\sim 92 \%$ of the time during the northern and southern hemisphere passes. Owing to its time-of-flight coincidence system, JADE-I can tolerate a significantly higher noise rate, up to $17 \mathrm{kHz} / \mathrm{cm}^{2}$.

2.5.3.2 JADE-E Figure 44 shows the results of a similar analysis for JADE-E, where we estimate the fraction of time that the JADE-E MCP background count rate will remain below $2 \mathrm{kHz}$ for each orbit if the sensor is shielded with a graded-Z shield with a stopping power of 

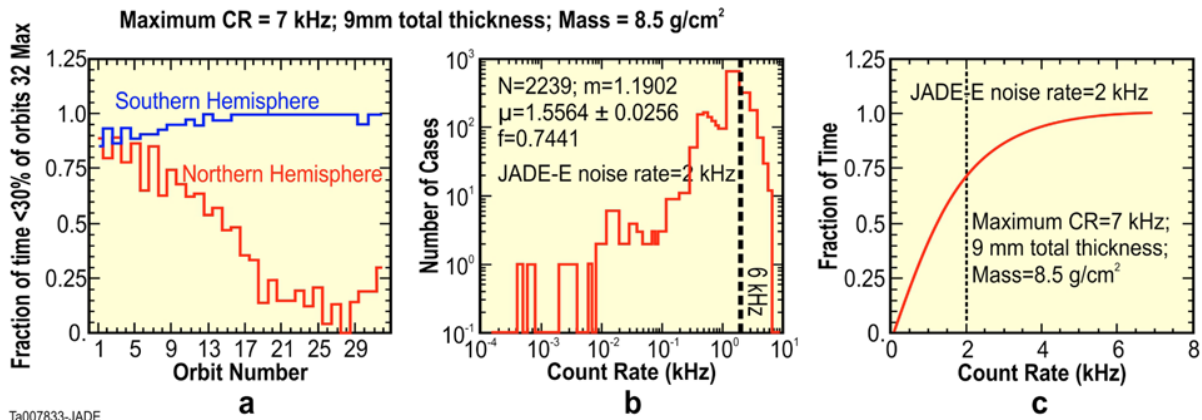

Fig. 44 Similar to Fig. 43, but for JADE-E and using a 9-mm thick shield with a stopping power of $8.5 \mathrm{~g} / \mathrm{cm}^{2}$

$8.5 \mathrm{~g} / \mathrm{cm}^{2}$ and $9 \mathrm{~mm}$ total thickness. Note that the peak background rate due to transmitted photons and electrons through this shield will result in a peak noise rate of $7 \mathrm{kHz}$ corresponding to the peak auroral flux of $\sim 2.2 \times 10^{6}$ at $1 \mathrm{MeV}$. The results are similar to those for JADE-I, and the third panel shows that the JADE-E background count rate will remain below $\sim 2 \mathrm{kHz} \sim 75 \%$ of the time during the northern and southern hemisphere passes.

\subsubsection{Shielding Modeling and Optimization}

Given the shielding numbers from Sect. 2.5.2 (5 and $8.5 \mathrm{~g} / \mathrm{cm}^{2}$ for JADE-I and JADE-E, respectively), we optimized the geometry of the shielding by examining all possible ray paths that intersect anywhere on the anode from any direction in space. To evaluate the shielding thickness we imported the mechanical design with our first estimate of the shielding into the NOVICE software to conduct the ray tracing. Then, we evaluated the amount of shielding in all directions and scaled up or down the thicknesses of the different shielding parts. We iterated between design modifications and ray tracing results until the shielding was reasonably optimized while maintaining a feasible mechanical design. In addition to minimizing background counting on the anodes, we also estimated the shielding thickness around the JADE pre-amplifiers, as these include parts, cannot exceed $50 \mathrm{kRad}$ TID (100 kRad with a Radiation Dose Margin (RDM) of 2) at the end of mission; this requirement translates to be $3.7 \mathrm{~g} / \mathrm{cm}^{2}$ around the pre-amplifiers.

Figure 45 shows a map of the shielding thickness for JADE-I looking in all directions around anode 6; similar maps were produced for the other anodes. The azimuth is the deflection angle of JADE-I. The polar angle at a given azimuth is the angle measured from the azimuthal plane. The results show that no direction has less than $5 \mathrm{~g} / \mathrm{cm}^{2}$ of shielding thickness. Many directions have much higher shielding thicknesses and there is a small gray area where the shielding exceeds $20 \mathrm{~g} / \mathrm{cm}^{2}$.

Figure 46 shows a similar map of the shielding thickness for JADE-E as viewed from anode 8. The elevation axis in this figure corresponds to the elevation axis of Fig. 6 but with the origin at the center of anode 8 . The polar axis is perpendicular to the elevation, and the polar angle is measured in a plane inclined at elevation.

There are a few small areas on the map where the shielding does not quite reach $8.5 \mathrm{~g} / \mathrm{cm}^{2}$. Although, it was not possible with the shielding mass available to cover all of these small areas, the fraction of the sky where the shielding is $<8.5 \mathrm{~g} / \mathrm{cm}^{2}$ is only $\sim 1.5 \%$. We calculated the impact these small areas have on the background rates using the results from Fig. 42 and integrating the background rate over the entire sky for each anode. The 
Fig. 45 Rectangular projection of the JADE-I radiation shielding thicknesses as viewed from Anode 6

Fig. 46 Rectangular projection of the JADE-E radiation shielding thickness viewed from Anode 8
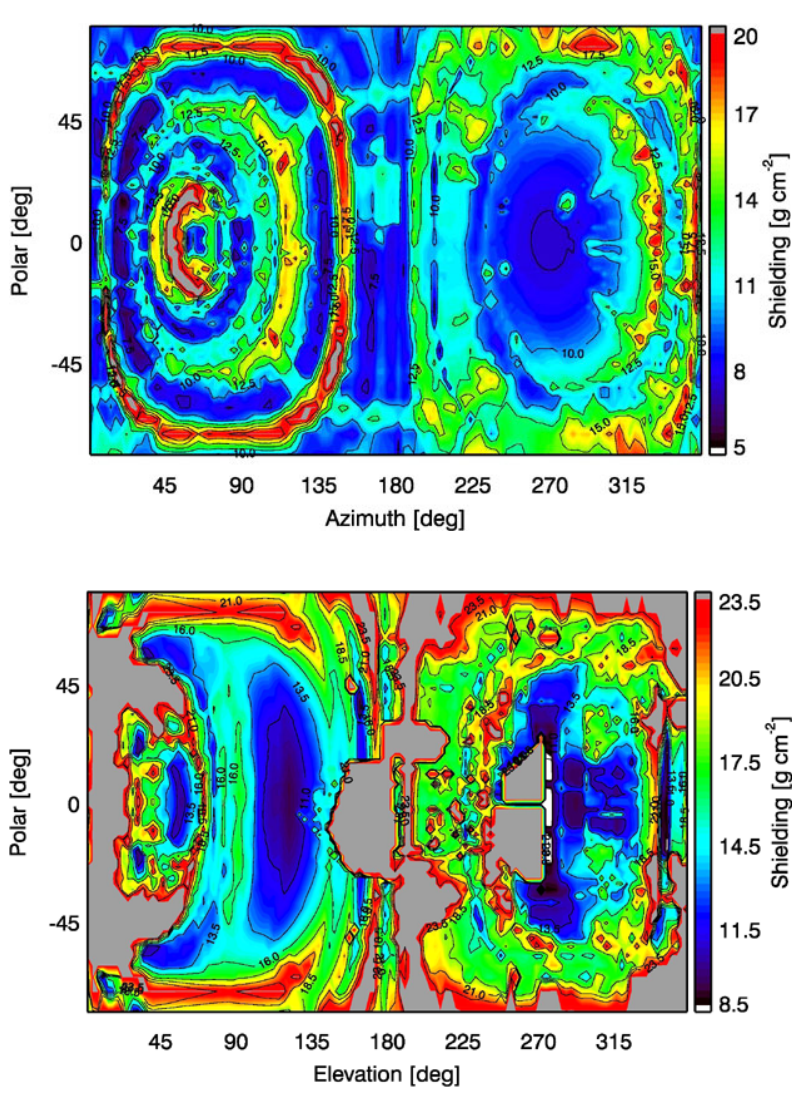

maximum rate on the worst and best anodes was $2.2 \mathrm{kHz}$ and $2.0 \mathrm{kHz}$, respectively, showing that the shielding is well-distributed and optimized for JADE-E.

\section{Calibration}

\subsection{Overview}

Calibration is critical for making precise and scientifically useful observations of space plasmas. Regrettably, some instruments encounter significant problems in development, allow the time available for calibration to become compressed, and never carry out full and detailed calibrations. In the case of JADE, we were especially concerned that the simultaneous production, testing, and calibration of the four sensors (JADE-I and three JADE-Es, which are essentially independent instruments) could stress the team's ability to hold schedule and maintain enough time for detailed calibrations. Thus, from the beginning, we scheduled additional time for calibration and started the calibrations very early-as soon as any portion of each sensor was available for testing. This approach had the added benefit of allowing intervals of both pre- and post-environmental test calibration, which further demonstrated that all aspects of the flight hardware survived the testing without degradation. In the end, both JADE-I and all three JADE-E sensors had ample time and were completely and fully calibrated as planned. 
Fig. 47 Sketch of the JADE-E calibration setup

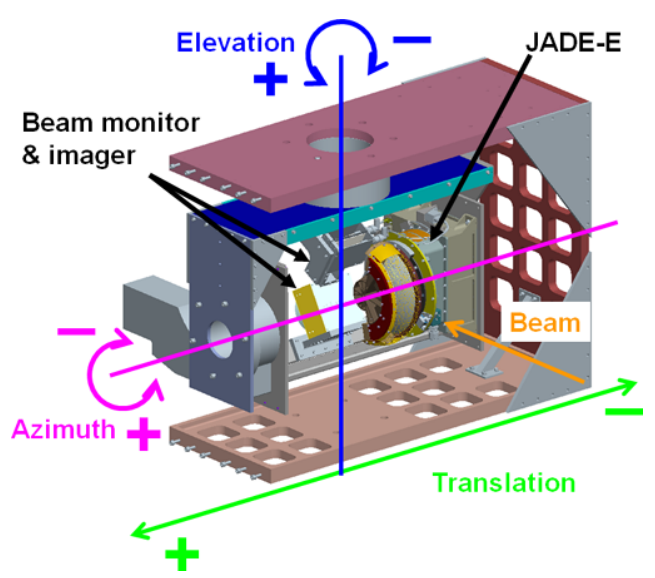

JADE-I was calibrated in the Southwest Research Institute ion instrument calibration facility (see Appendix in McComas et al. 2009), which was totally refurbished and expanded into a world-class facility starting in late 2000 . In order to fully and properly calibrate the three JADE-E sensors, we designed, built and validated a completely new electron calibration facility. The details of this new facility are described in the Appendix of this paper.

\subsection{JADE-E}

We built four nearly identical JADE-E sensors. Although all four showed very similar characteristics, the three with the best performance as demonstrated by their detailed calibrations are flying on Juno, with the fourth used on the ground for continuing additional testing as needed.

JADE-E sensors were calibrated at SwRI's new electron calibration facility (see the Appendix). The sensors were mounted on a three-axis, high-accuracy positioning system. Figure 47 shows an engineering sketch of the setup. The sensor can be moved with respect to the beam such that the entire FOV can be illuminated by the electron beam. The positioning system has two rotation axes, one for the azimuth and one for the elevation angle, as well as one translation axis along the azimuth axis. The electron beam can be steered left-right and up-down and focused or defocused. A beam imager and a beam monitor are mounted on the opposite side of the elevation axis so that a $180^{\circ}$ rotation around the elevation axis places these detectors in the beam line for its characterization.

The beam imager was equipped with a collimator on half of its sensitive area in order to determine accurately $\left(\sim 0.1^{\circ}\right)$ the beam direction at the location of the JADE-E aperture (its curved entrance grid). The beam direction and location was measured for all the energies used $(\sim 0.1$ to $30 \mathrm{keV})$. The beam intensity was measured using an absolute beam monitor $(\mathrm{ABM})$, which is based on a coincidence technique described by Funsten et al. (2005). A beam intensity measurement was taken before and after each test. If the test was longer than $\sim 30$ minutes, we also used a periodic normalization of the beam intensity using JADE-E in a particular setting, such as beam orientation and voltage configuration near the maximum transmission. The beam intensity was interpolated between the normalization points.

We compared our calibration measurements with simulations results. All electro-optics simulations were run using SIMION 8.0 or newer versions and set to reproduce the conditions (sensor orientation, beam shape, residual magnetic field, etc.) of the calibration chamber. The residual magnetic field was measured in a volume comparable to the sensor at its 
Table 9 Key property tests for top-hat analyzers and deflectors

\begin{tabular}{ll}
\hline Test & Measured \\
\hline $\begin{array}{l}\text { Voltage angle scans at different energies } \\
\text { and deflections }\end{array}$ & $\begin{array}{l}\text { Count rate (i.e., relative transmission) while scanning } \\
\text { elevation and voltage at fixed beam energy } \\
\text { Elevation scans at different energies }\end{array}$ \\
$\begin{array}{l}\text { Count rate (i.e., relative transmission) while scanning } \\
\text { elevation angle at different energies and fixed ESA voltage } \\
\text { and beam energy } \\
\text { Count rate (i.e., relative transmission) while scanning azimuth } \\
\text { angle at fixed voltage and beam energy }\end{array}$ \\
Background & $\begin{array}{l}\text { Count rate without electron beam or other stimulation } \\
\text { Count rate with UV source while scanning orientation } \\
\text { (elevation and azimuth) and voltages }\end{array}$ \\
UV response & $\begin{array}{l}\text { Count rate as a function of MCP voltage with constant input } \\
\text { beam }\end{array}$ \\
\hline
\end{tabular}

location in the vacuum chamber, with most of the field attributed to the Earth's magnetic field. Even though the vacuum chamber is comprised of non-magnetic materials, some elements (such as the motors of the positioning system) still carry a residual magnetic field. Measurements show these values reduce to background levels only a few centimeters away from the source.

Table 9 shows the series of tests we performed to determine the key properties of the top-hat analyzers and the deflectors.

We performed two calibration campaigns: pre-environmental testing calibration ("precal") and post-environmental testing calibration ("cal"). Results from both campaigns were compared to each other and matched very well, indicating that the environmental testing did not affect the calibrations. Our measurements were taken with two different electron sources (see the Appendix) to cover 0.1 to $30 \mathrm{keV}$, and the angular response was scanned at discrete orientations covering the entire JADE-E FOV. Despite the good angle and energy coverage, we did not explore the response beyond $30 \mathrm{keV}$ (maximum energy of our electron source), which is very close to the upper end of the energy requirement of $40 \mathrm{keV}$. This highest energy end can be reliably extended based on the measurements up to $30 \mathrm{keV}$ and the use of SIMION.

The sensors' responses were measured using the same high-voltage power supplies and beam, providing efficient and accurate comparisons. The JADE high-voltage power supplies and the electron source power supplies were calibrated using the same volt meter.

\subsubsection{Electro-Optics Characteristics}

3.2.1.1 Analyzer Constant The analyzer constant is the ratio of the mean kinetic energy per charge (one for electrons) selected by the analyzer to the voltage applied to the analyzer plate:

$$
k=\frac{E}{V}[\mathrm{keV} / \mathrm{kV}]
$$

In principle, this ratio is a constant for a given ESA and for non-relativistic energies. At the upper end of the JADE-E energy range, the non-relativistic assumption is no longer valid. The JADE-E analyzer constant deviates from Eq. (3.1). Figure 48 shows the measured analyzer constant from JADE-E pre-calibration, calibration, and simulations. A correction 
Fig. 48 Analyzer constant for the JADE-E sensors measured on anode 9 (near the FOV center)

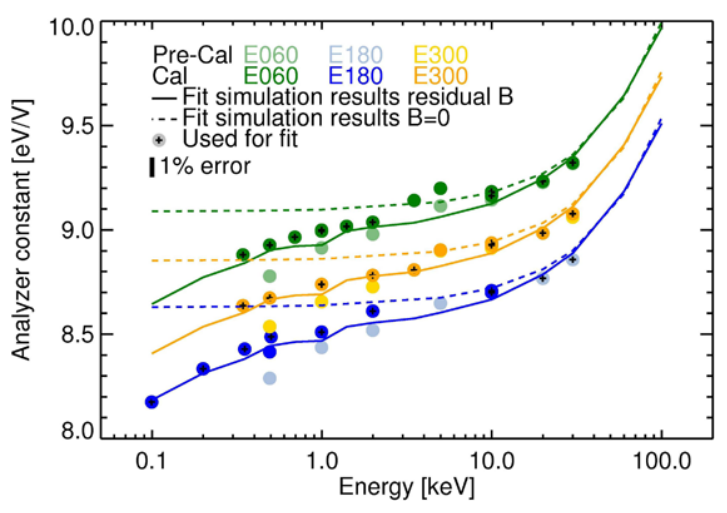

due to relativistic effects is necessary to account for the dependence on the energy of the analyzer constant. The analyzer constant can be related to the equation of motion in the analyzer.

$$
k=\frac{E}{V}=\frac{\frac{1}{2} m_{e} v^{2}}{V} \cong \frac{r}{2 \Delta r}
$$

where $m_{e}$ is the electron mass, $v$ is its speed, $r$ and $\Delta r$ are the mean radius and the gap between the electrodes of the analyzer, respectively. The relativistic kinetic energy, $E_{r}$, can be written as a function of the non-relativistic energy (Keski-Rahkonen and Krause 1978)

$$
E_{r}=E-m_{e} c^{2}+\sqrt{E^{2}+\left(m_{e} c^{2}\right)^{2}}
$$

where $c$ is the speed of light. After expansion into a power series we obtain

$$
E_{r}=E\left[1+\frac{1}{2} \frac{E}{m_{e} c^{2}}+O\left(\frac{E^{3}}{m_{e}^{3} c^{5}}\right)\right]
$$

Replacing $E$ by $E_{r}$ in Eq. (3.2) using Eq. (3.4) and neglecting the higher order terms, we find

$$
k(E) \cong \frac{E\left[1+\frac{1}{2} \frac{E}{m_{e} c^{2}}\right]}{V}=\frac{E}{V}+\frac{E}{V} \frac{E}{2 m_{e} c^{2}}
$$

Assuming that $E / V$ is constant we get the following expression for the analyzer constant

$$
k(E)=k_{0}+k_{1} E
$$

This relationship closely matches the response we obtained from our simulations with the following fit parameters

$$
k(E)=9.030+0.009081 \cdot E[\mathrm{keV}]
$$

Before we can use Eq. (3.6) to fit the calibration data, we need to take the Earth's magnetic field into account, because its effect is greater than $1 \%$ below $\sim 2 \mathrm{keV}$. We measured the magnetic field inside the vacuum chamber at the location of the sensors during calibration and took the average of multiple measurements in a volume comparable to the sensor's 
Fig. 49 Analyzer constant at $10 \mathrm{keV}$ as a function of anode number. Darker colors are for calibration and lighter ones are for pre-calibrations

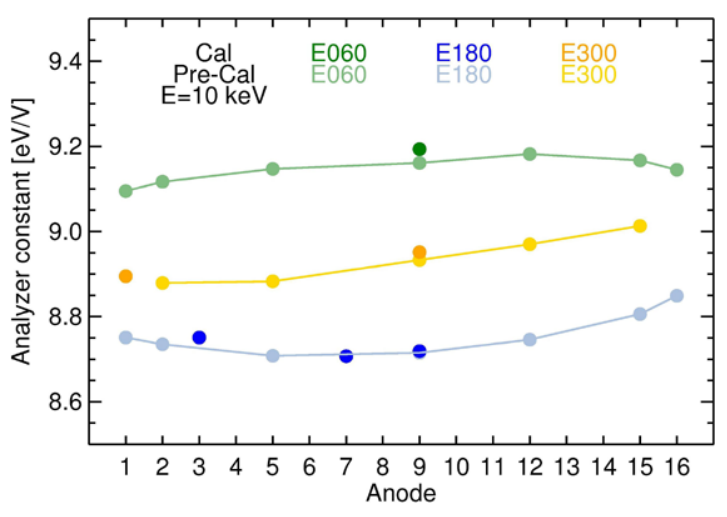

envelope for the simulations. The solid curves in Fig. 48 represent the results of these simulations with an offset such that the curves match the data set for each sensor. It is not surprising to see small differences between the sensors because fabrication tolerances and alignment mean that the JADE-E sensors are not quite identical. The offset values are

FM2: $\quad$ offset $=0.05824 \mathrm{keV} / \mathrm{kV}$

FM3: $\quad$ offset $=-0.4011 \mathrm{keV} / \mathrm{kV}$

FM4: $\quad$ offset $=-0.1784 \mathrm{keV} / \mathrm{kV}$

We assume that in the absence of magnetic field the offsets are the same, thus we can use those values to also offset the curve given by Eq. (3.7) (Fig. 48). Therefore, by adding the offset we obtain

$$
k(E)=9.030+0.009081 \cdot E[\mathrm{keV}]+\text { offset }
$$

where offset is the corresponding value from Eq. (3.8). Together, Eqs. (3.8) and (3.9) provide the analyzer constant as a function of energy for each sensor.

The differences between pre-calibration and calibration are 1-2\% at the lowest energies and become less than $1 \%$ above $1 \mathrm{keV}$. The pre-cal data deviate from the curves at low energies because the residual magnetic field changed between pre-calibration and calibration. We used only calibration data for the fits at the lowest energies.

Analyzer Constant Uniformity The gap between the ESA plates is critical for the analyzer constant. Since the mechanical alignment is not perfect, we expect differences across the FOV. We measured the analyzer constant at $10 \mathrm{keV}$ for at least 6 anodes for each sensor. Figure 49 shows the variations of $k(E=10 \mathrm{keV})$ across the azimuthal FOV.

The analyzer constant is within $\sim 1.6 \%$ of its value at anode 9 across the entire FOV, which means that the ESA electrodes are very nearly concentric. Table 10 provides more detailed information on the individual calibrated responses ( $k$ is given in units of $\mathrm{keV} / \mathrm{kV}$ ).

3.2.1.2 Energy Resolution We define the energy resolution of the analyzer as the FWHM divided by the most probable energy of a Gaussian fit to the energy passband. Figure 50 compares the energy resolution from pre-calibration and calibration measurements with simulations as a function of energy. The measurements were taken on anode 9 for each sensor. The energy resolution increases with energy for both the measurements and the simulations, but more rapidly for the measurements. Electrons reflect off surfaces inside the sensor (Sect. 3.2.1.5), and the higher the energy, the higher the reflectivity. Thus, electrons 
Table 10 Minimum, maximum, average of the analyzer constant at $10 \mathrm{keV}$

\begin{tabular}{|c|c|c|c|c|c|c|c|}
\hline Sensor & $k_{\min }$ & $k_{9}$ & $k_{\max }$ & $\begin{array}{l}\left(k_{9}-k_{\min }\right) / k_{9} \\
\%\end{array}$ & $\begin{array}{l}\left(k_{\max }-k_{9}\right) / k_{9} \\
\%\end{array}$ & Average & $\begin{array}{l}\text { Standard } \\
\text { deviation }\end{array}$ \\
\hline E060 & 9.095 & 9.161 & 9.182 & 0.72 & 0.23 & 9.145 & 0.030 \\
\hline E180 & 8.708 & 8.715 & 8.849 & 0.08 & 1.54 & 8.759 & 0.051 \\
\hline E300 & 8.879 & 8.933 & 9.013 & 0.60 & 0.90 & 8.936 & 0.057 \\
\hline
\end{tabular}

Fig. 50 Energy resolution (FWHM) as a function of energy. Darker shades are for calibrations and lighter shades are for pre-calibrations. See text for the difference between the simulations and the linear fits

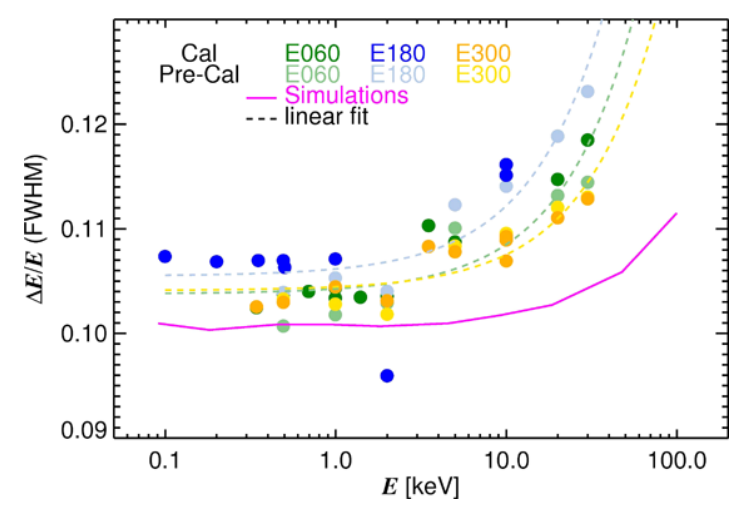

Table 11 Energy resolution fit coefficients and estimates

\begin{tabular}{llllll}
\hline Sensor & $a_{0}$ & $a_{1}[1 / \mathrm{keV}]$ & \multicolumn{2}{l}{$\Delta E / E$ estimates from the fits } & at $100 \mathrm{keV}$ \\
\hline E060 & & at $0.2 \mathrm{keV}$ & at $40 \mathrm{keV}$ & 0.123 & 0.151 \\
E180 & 0.104 & 0.000474 & 0.104 & 0.132 & 0.172 \\
E300 & 0.105 & 0.000673 & 0.106 & 0.118 & 0.139 \\
\hline
\end{tabular}

reflecting off the analyzer surfaces contribute to the tails of the energy distribution, which increase the FWHM. Here we use a linear fit to the pre-calibration and calibration data.

$$
\Delta E / E=a_{0}+a_{1} E
$$

where $E$ is given in $\mathrm{keV}$ and the fit coefficients are given in Table 11.

The minimum $(10.4 \%)$ and maximum $(13.2 \%)$ resolutions are well within the requirements $(5 \%<\Delta E / E<15 \%$ for energies between 0.2 and $40 \mathrm{keV})$. We measured the energy resolution at $10 \mathrm{keV}$ for the other anodes and found it to be relatively constant across the FOV, confirming the good alignment of the ESA electrodes. We found variations of only $0.31 \%$ for E060, $0.25 \%$ for E180, and $0.23 \%$ for E300.

3.2.1.3 Geometric Factor The geometric factor, which is a measure of the sensitivity, is defined as the integral of the transmission function, $T$, which depends on the look direction in the FOV, $\Omega$, the electron entrance location in the aperture area, $A$, and the energy, $E$ :

$$
G=\iiint T(\Omega, A, E) d \Omega d A \frac{d E}{E}
$$


Fig. 51 Product of the geometric factor with the electron detection efficiency for all three JADE-E sensors

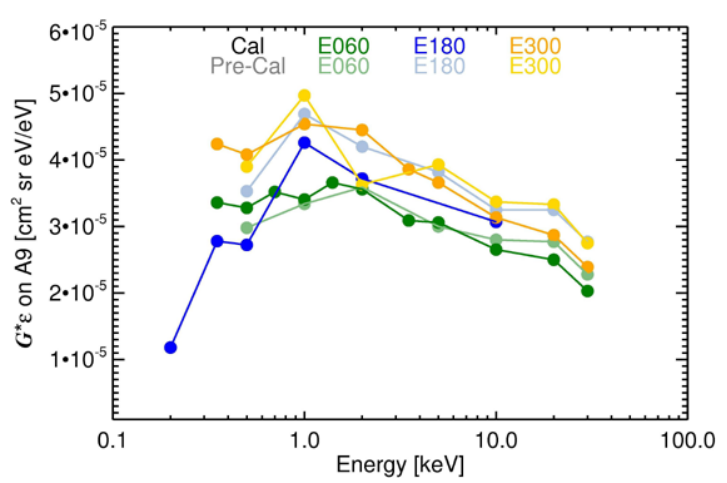

In practice we take discrete measurements and interpolate between them. Therefore, the previous equation can be rewritten as

$$
G \cong \sum_{j=1}^{n_{j}} \sum_{i=1}^{n_{i}} \cos \left(\delta_{j}\right) \Delta \delta \frac{R\left(\delta_{j}, V_{i}\right)}{\varepsilon F} \frac{\Delta V}{V_{i}} \Delta \varphi
$$

where the solid angle of the FOV is $\Omega_{j}=\cos \left(\delta_{j}\right) \Delta \delta \Delta \varphi$, the active area is $A_{i, j}=$ $R\left(\delta_{j}, V_{i}\right) / \varepsilon F$, and the energy resolution is $\Delta E / E_{i}=\Delta V / V_{i} . \delta_{j}$ is the incident direction of the electron in the deflection plane when they enter the deflectors region, and $\Delta \delta$ is the step size of the discrete measurements in angle. $\Delta \varphi$ is defined as the anode pixel angular width (FWHM $=7.5^{\circ}$ per pixel), and since the top-hat design has a cylindrical symmetry it comes out of the integral (or sum) and becomes simply a multiplication factor. $R\left(\delta_{j}, V_{i}\right)$ is the event rate measured on the detector in count $\mathrm{s}^{-1}, \varepsilon$ is the detection efficiency of the detector, and $F$ is the beam intensity in $\mathrm{cm}^{-2} \mathrm{~s}^{-1} . \Delta V$ is step size of the discrete measurements in voltage, and $\Delta \delta$ and $\Delta V$ are constant.

It would have been convenient to separate the detector efficiency, $\varepsilon$, which may vary over time, from the geometric factor, which is a property of the sensor and does not change over time. However, it is very difficult to obtain an absolute calibration of the detector efficiency. Instead, we measured the product of the geometric factor with the detector efficiency as shown in Fig. 51 for all three sensors as a function of energy. The product, $G \cdot \varepsilon$, reaches a maximum around $1 \mathrm{keV}$ and decreases slowly by a factor of $\sim 2$ at $30 \mathrm{keV}$. The variations in $G \cdot \varepsilon$ as a function of energy are mostly due to the energy dependent electron detection efficiency. Our simulations show a $\sim 4 \%$ increase of $G$ from 0.1 to $100 \mathrm{keV}$.

Because the FOV is slightly narrower when using the deflectors, the geometric factor decreases accordingly, as illustrated in Fig. 52 for six deflections ranging from $-29^{\circ}$ to $+33^{\circ}$ at $10 \mathrm{keV}$. The difference between the minimum and the maximum is $\sim 30 \%$ to $\sim 33 \%$ for all sensors at $0^{\circ}$ deflection.

3.2.1.4 Angular Resolution We considered three criteria in defining the anode number: (1) each JADE-E sensor has anodes from 1 to 16, (2) all three JADE-E sensors are seen as a "single sensor" that has anodes from 1 to 48 where E060 has anodes from 1 to 16, E180 from 17 to 32, and E300 from 33 to 48, and (3) same as (2) with anode 1 having the same look direction as the magnetic field vector. The first definition allows easy comparison from sensor to sensor, but the JADE-E sensor name has to be used in order to uniquely identify a look direction on the spacecraft; for example anode 1 in E060 has a look direction $120^{\circ}$ away from anode $1 \mathrm{in}$ E180 or E300. Note that the center of the azimuthal FOV is at $60^{\circ}$ for 
Fig. 52 Geometric factor times detector efficiency as a function of deflection angle for $10 \mathrm{keV}$ on anode 9 . We also measured the geometric factor for other anodes without deflection and at $10 \mathrm{keV}$. The response across the anodes is uniform within $13 \%$ (excluding anode 1 and 16)

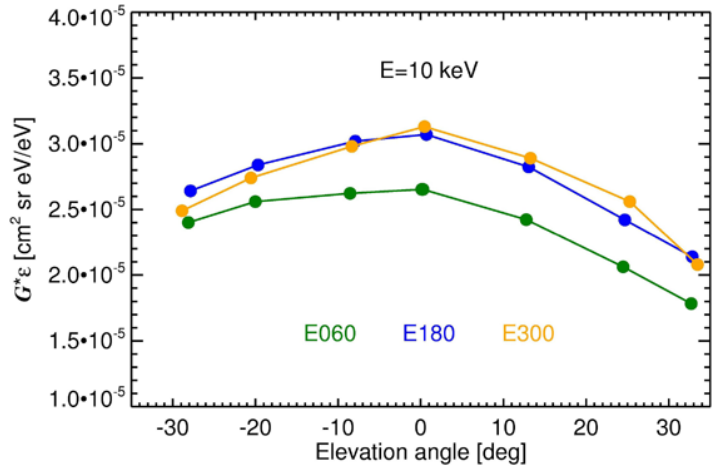

Table 12 Azimuth angle of anode centers

\begin{tabular}{lllllllll}
\hline Anode & 1 & 2 & 3 & 4 & 5 & 6 & 7 & 8 \\
\hline Azimuth $\left[^{\circ}\right]$ & 3.75 & 11.25 & 18.75 & 26.25 & 33.75 & 41.25 & 48.75 & 56.25 \\
\hline \hline Anode & 9 & 10 & 11 & 12 & 13 & 14 & 15 & 16 \\
\hline Azimuth $\left[{ }^{\circ}\right]$ & 63.75 & 71.25 & 78.75 & 86.25 & 93.75 & 101.25 & 108.75 & 116.25 \\
\hline
\end{tabular}

$\mathrm{E} 060,180^{\circ}$ for $\mathrm{E} 180$, and $300^{\circ}$ for $\mathrm{E} 300$ in spacecraft coordinates. The second definition has the advantage of a unique anode number for a particular direction but is fixed with respect to the spacecraft coordinates. The third definition is mostly used to collapse the burst data (full angle and energy resolution) into the high rate science (HRS) data format.

The azimuth angle discussion below uses the first definition of the anode numbers, with the anode center look directions and assuming that the azimuthal FOV ranges from $0^{\circ}$ to $120^{\circ}$ (Table 12).

Azimuthal Angle We measured the azimuthal response by scanning angles in the imaging plane in small $\left(0.75^{\circ}\right)$ steps across the FOV. Adjustments to the elevation angle and ESA voltages were required to account for the slight misalignments due to the tolerances of the mounting fixture and ESA plates. The count rate for a constant beam as a function of azimuth is shown in Fig. 53 for all three sensors. In general, the response is uniform to within $20 \%$ for all anodes. Anodes 1 and 16 are at the edge of the FOV, which is why the curves have different shapes and the integral of the counts is slightly lower compared to other anodes. A reduction in transmission at the edge of the FOV is expected. The azimuthal angle resolution is $\sim 7.5^{\circ}$ (average) for all three sensors, matching our goal and fully half of the maximum requirement of better than $15^{\circ} \mathrm{FWHM}$.

Elevation Angle We measured the elevation angle response when the deflectors are biased at a given voltage by scanning elevation in $0.5^{\circ}$ steps. We also repeated the elevation scans for different voltages applied to the upper and lower deflectors. An example of the deflector response is shown in Fig. 54. The colors correspond to various deflector voltage settings (indicated at the top). This particular set of measurements was taken with a $10 \mathrm{keV}$ beam on anode 9 . The deflection range is roughly $-35^{\circ}$ to $+35^{\circ}$. We integrated the total number of counts (per unit of time) for each of the distributions shown in the figure and plotted this number (red diamonds) according to the scale on the right. The total counts per deflection setting is roughly constant (within $\sim 10 \%$ of the maximum) with a small decrease $(\sim 15$ to $35 \%)$ beyond $+30^{\circ}$. 
Fig. 53 Azimuthal angle response of all JADE-E sensors. (Note that there are no beam changes during calibration. Downward trend can be attributed to beam misalignment during sensor rotation; slight, off-transmission-peak variation ESA voltage; different efficiencies of MCP; or differences in FEE)

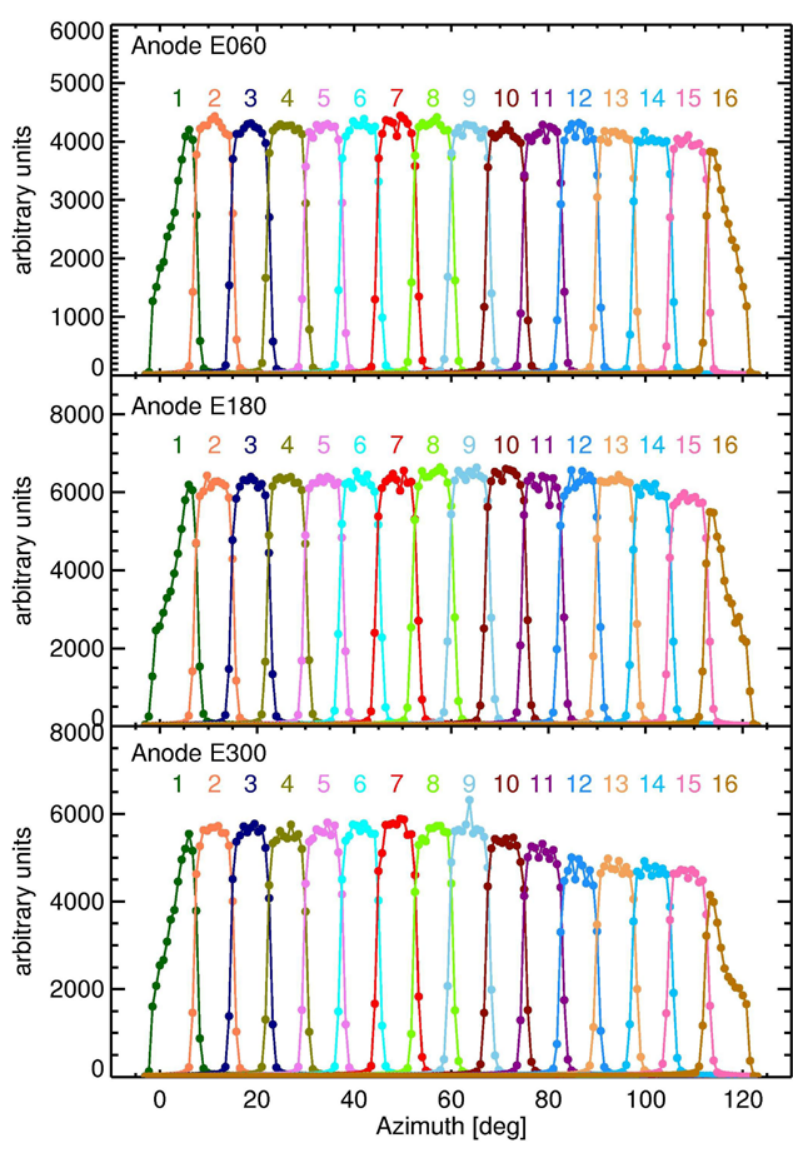

Fig. 54 Example of a deflection scan for different voltages (indicated at the top) applied to the upper and lower deflectors. The relative transmission is shown with red diamonds, with the scale on the right

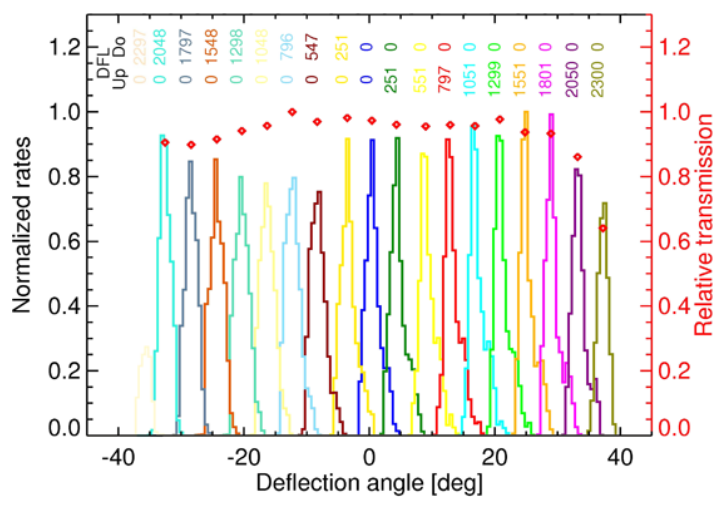

Taking the most probable values of Gaussian fits to the distributions in Fig. 54, we obtain the deflection angles and plot them versus deflector voltage divided by the energy in Fig. 55 . The relationship is very nearly linear, but is slightly better represented with a 3rd order polynomial fit. 
Fig. 55 Mean deflection angle as a function of deflector voltage. The deflector voltage is defined positive for positive elevation and negative for negative elevation even though both the upper and lower deflectors are biased positive

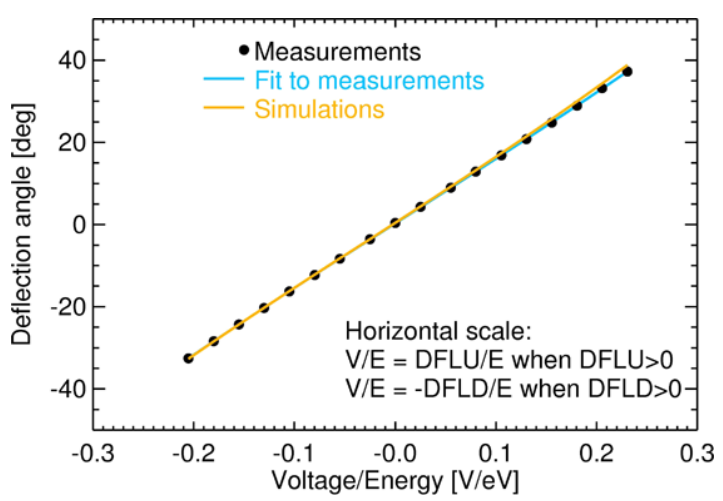

Table 13 Deflection coefficients

\begin{tabular}{llllc}
\hline JADE sensor & $\varepsilon_{0}\left[{ }^{\circ}\right]$ & $\varepsilon_{1}[\%(\mathrm{~V} / \mathrm{eV})]$ & $\varepsilon_{2}\left[\%(\mathrm{~V} / \mathrm{eV})^{2}\right]$ & $\varepsilon_{3}\left[\% /(\mathrm{V} / \mathrm{eV})^{3}\right]$ \\
\hline E060 & -0.24 & 0.71 & -3.65 & 18.0 \\
E180 & -0.37 & 0.20 & -3.30 & -3.34 \\
E300 & -0.23 & 4.65 & -5.81 & -13.7 \\
\hline
\end{tabular}

We define the deflection angle as follows

$$
\delta=a_{0}+a_{1}\left(\frac{V}{E}\right)+a_{2}\left(\frac{V}{E}\right)^{2}+a_{3}\left(\frac{V}{E}\right)^{3}
$$

where $V[\mathrm{kV}]$ is the deflector voltage ( $V<0$ if DFL-DN is powered, $V>0$ if DFL-UP is powered), $E[\mathrm{keV}]$ is the electron energy, and the $a_{i}(i=0, \ldots, 3)$ are the deflection coefficients

$$
\begin{aligned}
& a_{0}=-0.11+\varepsilon_{0} \\
& a_{1}=154.85+1.582 \cdot 10^{-4} E+\varepsilon_{1} \\
& a_{2}=-2.457+\varepsilon_{2} \\
& a_{3}=52.02+\varepsilon_{3}
\end{aligned}
$$

The $\varepsilon_{i}(i=0, \ldots, 3)$ are different offsets for each sensor. We define $a_{0}$ as the deflection offset and $a_{1}$ as the deflection constant because it is the term that expresses the proportionality between the deflection angle and the voltage-to-energy ratio. The coefficients $a_{2}$ and $a_{3}$ are higher order terms. The deflection coefficients for all three sensors are given in Table 13.

The deflection offset is a simple look direction offset in the elevation angle. The deflection constant roughly determines the deflection for a particular voltage/energy ratio and depends on the energy, similarly to the analyzer constant. The deflection constant is roughly uniform across the azimuthal FOV. The minimum and maximum values for $a_{1}$ are:

- E060: $\min 156.0, \max 158.1^{\circ} /(\mathrm{V} / \mathrm{eV})$

- E180: $\min 154.7, \max 156.6 \%(\mathrm{~V} / \mathrm{eV})$

- E300: $\min 158.4, \max 163.7^{\circ} /(\mathrm{V} / \mathrm{eV})$

For example, at $1 \mathrm{keV}$ and $200 \mathrm{~V}$ on DFL-UP, the minimum and maximum deflection angles across the azimuthal FOV would be: 
Table 14 Reverse relationship deflection coefficients

\begin{tabular}{lllllr}
\hline JADE sensor & $\gamma_{0}$ & $b_{10}$ & $b_{11}$ & $\gamma_{2}$ & $\gamma_{3}$ \\
\hline E060 & 0.00158 & $6.425 \mathrm{E}-3$ & $-5.73 \mathrm{E}-6$ & $7.62 \mathrm{E}-7$ & $-2.46 \mathrm{E}-8$ \\
E180 & 0.00237 & $6.448 \mathrm{E}-3$ & $-5.87 \mathrm{E}-6$ & $7.40 \mathrm{E}-7$ & $6.15 \mathrm{E}-9$ \\
E300 & 0.00145 & $6.268 \mathrm{E}-3$ & $-5.61 \mathrm{E}-6$ & $1.29 \mathrm{E}-6$ & $2.97 \mathrm{E}-8$ \\
\hline
\end{tabular}

- E060: $\min 31.2^{\circ}, \max 31.6^{\circ}$

- E180: $\min 30.9^{\circ}, \max 31.3^{\circ}$

- E300: $\min 31.7^{\circ}, \max 32.7^{\circ}$

At most, this is $\sim 1^{\circ}$ for a deflection close to the maximum. This effect has been calibrated, to be accounted for in the data analysis.

JADE receives the magnetic field vector information from the spacecraft and looks up a deflection angle (elevation) in a lookup table that contains the deflector voltage for each ESA voltage; this table provides a deflection angle equal to the elevation angle of the magnetic field vector. Thus, we need to transform Eq. (3.13) and express the voltage as a function of the energy and deflection angle

$$
V=E \cdot\left(b_{0}+b_{1} \delta+b_{2} \delta^{2}+b_{3} \delta^{3}\right)
$$

where $V$ is the voltage in $\mathrm{kV}, E$ is the electron energy in $\mathrm{keV}, \delta$ is the elevation angle in degrees, and the $b_{i}(i=0, \ldots, 3)$ are the reverse relationship deflection coefficients:

$$
\begin{aligned}
& b_{0}=7.16 \cdot 10^{-4}+\gamma_{0} \\
& b_{1}=b_{10}+b_{11} E \\
& b_{2}=5.85 \cdot 10^{-7}+\gamma_{2} \\
& b_{3}=-8.51 \cdot 10^{-8}+\gamma_{3}
\end{aligned}
$$

The $\gamma_{i}(i=0, \ldots, 3)$ are different offsets for each sensor (Table 14).

The maximum deflection is obtained when the deflector voltage is at its highest value $(+10 \mathrm{kV})$. For $100 \mathrm{keV}$ electrons the maximum deflection is $\sim 16^{\circ}$, and $30^{\circ}$ is achievable for $<50 \mathrm{keV}$ electrons.

The beam from our electron source can be approximated by a point source, which implies that the response is different from that obtained with a parallel beam. This affects the elevation angle distribution, and particularly its width. We compared the calibration measurements with simulation results, taking into account the exact geometry of the calibration (source distance to JADE-E aperture, orientation and position of JADE-E with respect to the beam, etc.). Figure 56 shows this comparison for $10 \mathrm{keV}$ electrons at $\sim 0^{\circ}$ elevation. These two distributions, as well as others taken at larger deflection angles, agree very well thus validating our electro-optics model for deflections. From the simulations, we have $\sim 3$ to $6^{\circ}$ FWHM angular resolution for a parallel beam of electrons. The resolution is significantly better than the $15^{\circ}$ requirement.

3.2.1.5 Electron Reflections Electrons easily scatter off internal surfaces, which modify the electron trajectories and generates spurious counts because some electrons hit electrodes outside the ESA's energy-angle acceptance. We used mitigation strategies, including an Ebonol-C coating on the ESA electrodes, which also significantly attenuates UV photons 
Fig. 56 Comparison between calibration data and simulation results for the elevation angle response without deflectors
Fig. 57 Ratio of signal on anode 9 , for a beam centered on anode 9 , divided by the sum of the signal on all anodes. A ratio below 1 means that some of the signal is received on a different anode
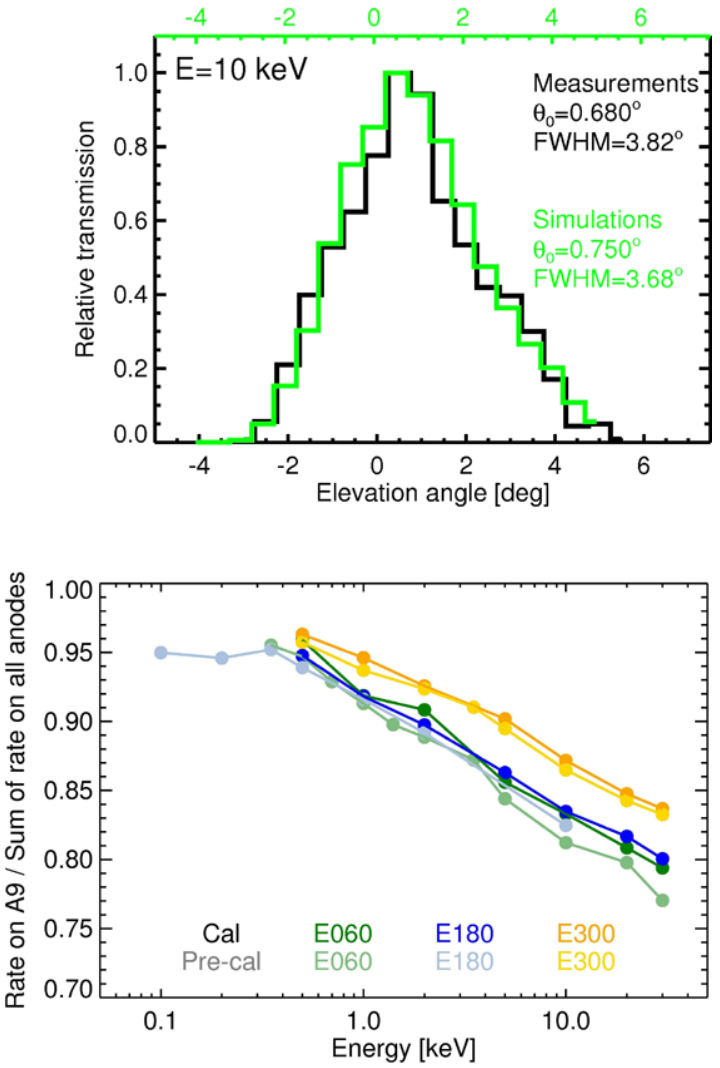

from reflecting deep into the sensor. Nevertheless, we observed some electron reflection as shown in Fig. 57. In that figure, the ratio of the signal for a beam centered on anode 9 to the signal on all anodes is plotted versus electron incident energy. Ideally, the ratio is 1 , that is, all the electrons fall on anode 9 . A value of 0.9 means that about $90 \%$ of the total signal is measured on anode 9 and $\sim 10 \%$ on neighboring anodes. The percentage of reflected electrons is $\sim 5 \%$ below $0.5 \mathrm{keV}$ and increases with energy up to $\sim 20 \%$ at $30 \mathrm{keV}$. This effect is most pronounced in the center of the azimuthal FOV and decreases at the edges. For example, at $10 \mathrm{keV}$ on anode 9 the percentage reflected is roughly 13 to $17 \%$, and it is only $\sim 6$ to $9 \%$ when the beam is directed toward anode 1 or 16 . Once quantified as we have done here, the reflected signal can be accounted for and removed in the data analysis.

\subsubsection{Response in a Magnetic Field}

Our electron calibration facility is equipped with three sets of coils to create a controlled magnetic field inside our vacuum chamber up to about 9 Gauss in any direction (see facility details in the Appendix). Before calibration, we used these coils to perform preliminary tests with the JADE-E engineering unit. In particular, we measured JADE-E's response when the magnetic field vector is along or perpendicular to the beam line. While these results showed that the agreement between the measurements and the simulations is good, additional testing on the fourth JADE-E sensor still on the ground is planned as a way to complete validation of the model. 
Table 15 Background rates in the laboratory

\begin{tabular}{llllll}
\hline Sensor & $\begin{array}{l}\text { Min. rate } \\
{[\mathrm{Hz}](\text { anode })}\end{array}$ & $\begin{array}{l}\text { Max. rate } \\
{[\mathrm{Hz}](\text { anode) }}\end{array}$ & $\begin{array}{l}\text { Average rate } \\
{[\mathrm{Hz}]}\end{array}$ & $\begin{array}{l}\text { Background anode } \\
{[\mathrm{Hz}]}\end{array}$ & $\begin{array}{l}\text { ESA voltage } \\
{[\mathrm{V}]}\end{array}$ \\
\hline E060 & $0.17(\mathrm{~A} 15)$ & $0.47(\mathrm{~A} 1)$ & 0.21 & 0.25 & 0,100 \\
E180 & $0.16(\mathrm{~A} 4)$ & $0.50(\mathrm{~A} 7)$ & 0.24 & 0.68 & $0,100,1090$ \\
E300 & $0.21(\mathrm{~A} 12)$ & $0.75(\mathrm{~A} 16)$ & 0.41 & 0.70 & 1070 \\
\hline
\end{tabular}

\subsubsection{UV Sensitivity}

The sensors were exposed to a strong UV source (equivalent to $\sim 1.4$ times the solar Lymanalpha at $1 \mathrm{AU}$ ) while we measured the rates and varied the orientation of each JADE-E sensor. The highest rates measured were $806 \mathrm{~Hz}$ for E060, $859 \mathrm{~Hz}$ for E180, and $2750 \mathrm{~Hz}$ for E300 when the ESA voltage was $\sim 10 \mathrm{~V}$. The rates decreased when ESA voltage increased, with rates in the few tens of $\mathrm{Hz}$ (or less) by the time the voltage was at $\sim 100 \mathrm{~V}$. The ESA voltage during science operations is always $\geq \sim 11 \mathrm{~V}$ (which corresponds to $\sim 100 \mathrm{eV}$ ). Photoelectrons may have entered the ESA because of the magnetic field, however, they must be accelerated beyond $\sim 12 \mathrm{eV}$ to cross the electron suppressor (SE sup) grid in front of the MCP (Sect. 2.2.1 and Fig. 7).

During the mission, we expect the reflected solar Lyman-alpha from Jupiter's atmosphere to be more than 40 times lower than the solar UV at 1 AU. The UV intensity from Jupiter's aurorae should be even less than the reflected solar UV. Therefore, by dividing the worst case by 40 , we obtain an upper limit of $\sim 75 \mathrm{~Hz}$ even for the very lowest energy measurements.

\subsubsection{Background in the Laboratory}

We measured the background rates in the laboratory without stimulation (no electron beam, no light) for extended periods. Table 15 summarizes the results of these tests. The rates are well under $1 \mathrm{~Hz}$ for all anodes. Different voltage settings on the ESA changed the background rates and backgrounds in space may be slightly higher (typically $2-3 \mathrm{~Hz}$ per anode) due to unshielded penetrating radiation such as cosmic rays; all of these are very low background values, enabling high quality observations from all three JADE-E sensors.

\subsection{JADE-I}

The JADE-I sensor was calibrated in the SwRI ion calibration facility (see Appendix in McComas et al. 2009) using ions over the energy range from $\sim 500 \mathrm{eV}$ to $50 \mathrm{keV}$ and of masses from $1 \mathrm{amu}$ (protons) to $40 \mathrm{amu}$ (Ar). The ion beam is monitored primarily with two sensors: an ABM that uses the coincidence method described by Funsten et al. (2005) to measure the absolute flux into the sensor, and a Quantar Imager that uses a large area $(100 \times 80 \mathrm{~mm})$ to measure the uniformity of the beam.

The JADE-I sensor is mounted on a 4-axis positioning system. Each of its linear translations, vertical motion and horizontal motion perpendicular to the incident beam line, is measured to better than $0.01 \mathrm{~mm}$ resolution. Two rotation stages, each with a resolution of $0.001^{\circ}$, have rotational axes that cross through the center of the JADE-I aperture. Figure 58 shows the JADE-I sensor mounted in the vacuum chamber for the flight calibration. The ion beam enters the chamber from the left in this photo.

As with JADE-E, the calibration of the JADE-I sensor was performed both before and after instrument level environmental testing to verify that performance was unchanged. All 
Fig. 58 The JADE-I sensor mounted for calibration on a 4-axis positioning system, which allows illumination of the full FOV

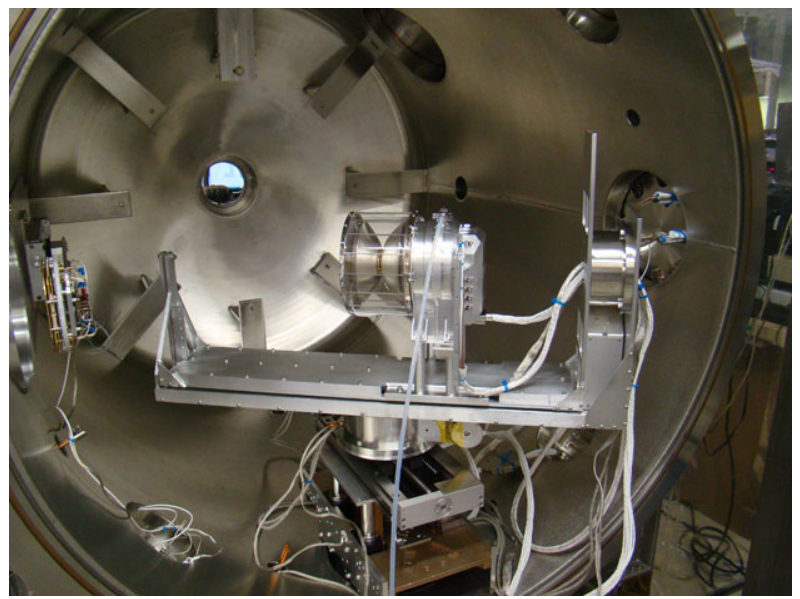

results shown in this section are from the post-environmental calibration as there was no observed change in performance between pre- and post-environmental testing. A detailed SIMION model of the electro-optics is in good agreement with the calibration results. The SIMION modeling consisted of $2.6 \times 10^{6}$ ions launched into the JADE-I electro-optics for each of the 32 nominal ESA voltage settings. The TOF section was set at its nominal value of $-10 \mathrm{kV}$, and the deflectors were set at $0 \mathrm{~V}$. Figures 59 and 64 show the SIMION-derived analyzer properties and the geometric factor, respectively. The geometric factor includes only the particles that would pass through the carbon foil assuming $100 \%$ transmissive grids and foils.

\subsubsection{Electro-Optics Characteristics}

3.3.1.1 Analyzer Constant as a Function of Energy and Pixel The analyzer constants for JADE-I are determined similarly to those of JADE-E (Sect. 3.2.1.1). However, many of the perturbations do not need to be accounted for in the ion sensor due to the much larger mass of ions $\left(m_{p} / m_{e}=1836\right)$ and, for example, relativistic and magnetic effects are not included for JADE-I.

A more significant effect on the ESA response is the TOF pre-acceleration described in Sect. 2.3. Modeling shows that the analyzer constant, energy resolution, and azimuthal angular response all have some energy dependence. Figure 11 shows how the focal point of the ESA changes as the ESA voltage increases. The analyzer constant (Eq. (3.1)) also has a weak energy dependence. Over the full energy range of the instrument ( $10 \mathrm{eV}$ to $45 \mathrm{keV})$, modeling shows the analyzer constant varies from 4.41 at $10 \mathrm{eV}$ to 4.36 at $40 \mathrm{keV}$, with most of the variation occurring between 10 and $200 \mathrm{eV}$ (see top panel of Fig. 59). Field penetration into the ESA effectively "shortens" the ESA. Particles receive an additional pull from the TOF, changing their trajectories and allowing those that would have impacted the end of the ESA to pass through to the TOF section.

Additional effects of the ESA shortening include energy-dependent energy resolution, azimuthal angular resolution, and offset of the non-deflected azimuthal zero. SIMION modeling predicts that the energy resolution of the analyzer, $\Delta E / E$, varies from $28 \%$ at $10 \mathrm{eV}$ to $19 \%$ at $40 \mathrm{keV}$ (Fig. 59). The azimuthal angular resolution varies by nearly $1.5^{\circ}$ and the offset of the FOV by $0.3^{\circ}$ over the full energy range of the sensor. 
Fig. 59 Electrostatic Analyzer (ESA) parameters as a function of Energy as determined from SIMION modeling. The penetrating electric field from the TOF results in effectively shortening the ESA for low energy particles
Fig. 60 Angular response of the analyzer constant. The measured analyzer constants for $\mathrm{He}^{+}$ (star), $\mathrm{N}_{2}^{+}$(diamond) and $\mathrm{Ar}^{+}$ (cross) for energies of $10 \mathrm{keV}$ are plotted in red and $40 \mathrm{keV}$ in blue. $8 \mathrm{keV} \mathrm{H}^{+}$data is plotted with green triangles
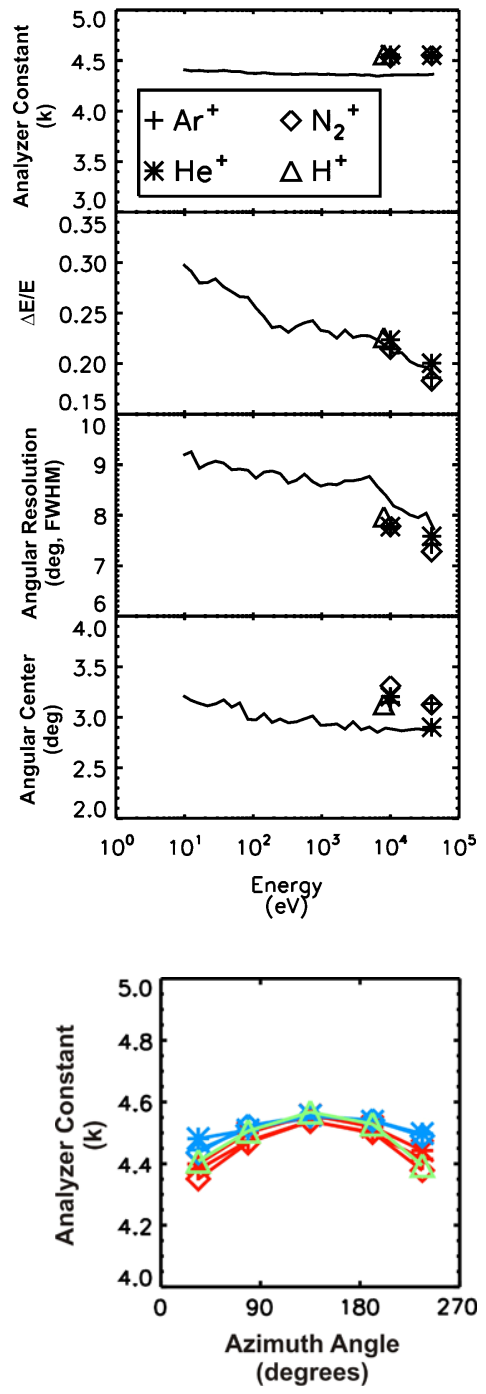

The calibration data agrees well with the model results. In Fig. 60 the measured values for the flight unit for 10 and $40 \mathrm{keV}$ are plotted for three species: $\mathrm{He}^{+}$(stars), $\mathrm{N}_{2}^{+}$(diamonds), and $\mathrm{Ar}^{+}$(crosses). The measured values differ by $\sim 5 \%$ from the modeled values.

Angular resolution is determined by the intrinsic response of the electro-optics, angular scattering of particles as they pass through the carbon foils, and the anode pad size, which corresponds to $22.5^{\circ}$. As the size of the electron pulse from the MCPs increases, the charge deposited on a given anode can also capacitively couple to its nearest neighbors. This is seen in the increased number of simultaneous adjacent and non-adjacent anodes. This effect helps define an optimal gain for the MCPs to be large enough to be sensitive to incident events, but not so large as to couple to the nearest neighbors.

The constant post-ESA acceleration has a varying effect on the different energy ions. When the ions exit the ESA they are accelerated toward the TOF in a direction normal to the carbon foils. For the lowest energy ions, the energy gained during the post acceleration is 


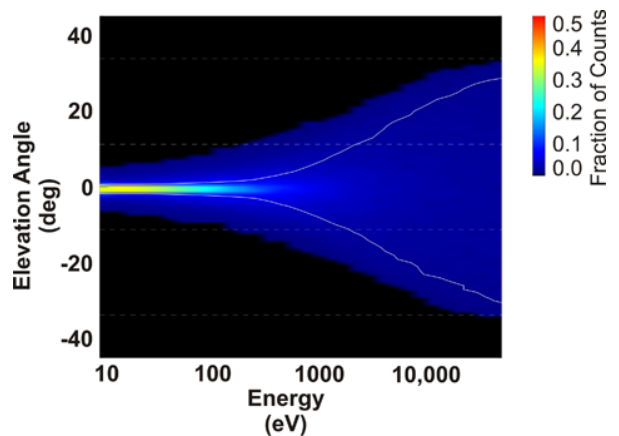

Fig. 61 Modeled distributions of the elevation angles as a function of energy. In this model, no angular scattering or energy straggling effects were included. Also it was assumed that all ions pass through the foils with a neutral charge state. Since including realistic scattering and electronic effect will further scatter the ions paths, this result should be interpreted as a limiting case of the angular response

significantly larger than their incident energy, and they consequently impact the foil normal to its surface. As the energy increases, the post acceleration has a smaller overall effect on the ions' trajectories. The higher energy ions can impact the foils at larger angles depending on how they exit the ESA.

Without scattering from grid or foil interactions, the highest elevation angular resolution is at the lowest energies. Figure 61 shows how the idealized angular resolution varies with energy in the absence of any scattering. The distribution of events is normalized for each energy step. The dashed lines show the anode spacing, and the solid white line shows the location of the FWHM. For this SIMION modeling run, all ion grids and foils are assumed to have $100 \%$ transmission. It is also assumed that the carbon foil does not cause any angular scattering or energy straggling for the ions, and all ions exit the carbon foils with a neutral charge. Including scattering and other effects broadens the angular width so the result presented in Fig. 61 is a limiting case of the angular resolution.

The carbon foils add a mass and energy dependent scattering to the system. For higher masses and lower energies, the magnitude of the scattering increases (Funsten et al. 1993). The $10 \mathrm{keV}$ post acceleration reduces the amount of scattering for the lowest incident energy ions. Without post acceleration, the lowest energy ions $(<100 \mathrm{seV})$ cannot even pass through the foil. Those low energy ions able to pass through have significant scattering ( $\sim 10 \mathrm{~s}$ of degrees). The measured angular resolution in the elevation direction (plotted in Fig. 62) includes all of the effects discussed above.

The JADE-I deflector plates scan $\pm 45^{\circ}$ for energies up to $24 \mathrm{keV}$, and up to $\pm 25^{\circ}$ at $40 \mathrm{keV}$. The linearity of the deflectors is shown in Fig. 63 for an $8 \mathrm{keV}$ proton beam. The voltage required to deflect an angle $\theta$ (in degrees) and energy $E$ (in $\mathrm{eV}$ ) is

$$
\begin{aligned}
& V_{D}=\frac{(\theta-\delta) E}{106.5} \\
& V_{U}=\frac{(\theta-\delta) E}{107.5}
\end{aligned}
$$

The factor $\delta$ is the offset angle of the FOV center and is plotted in Fig. 59. Since a positive potential is applied to the deflector plates, the up deflector is used to look downward, and vice versa. 
Fig. 62 Elevation angular resolution as function of energy for the 12 anodes. This data was taken at $2300 \mathrm{~V}$ on the MCP, which will have an increased cross talk between anodes
Fig. 63 Required deflection voltage for $8 \mathrm{keV}$ protons where different deflectors are used to deflect in the two directions
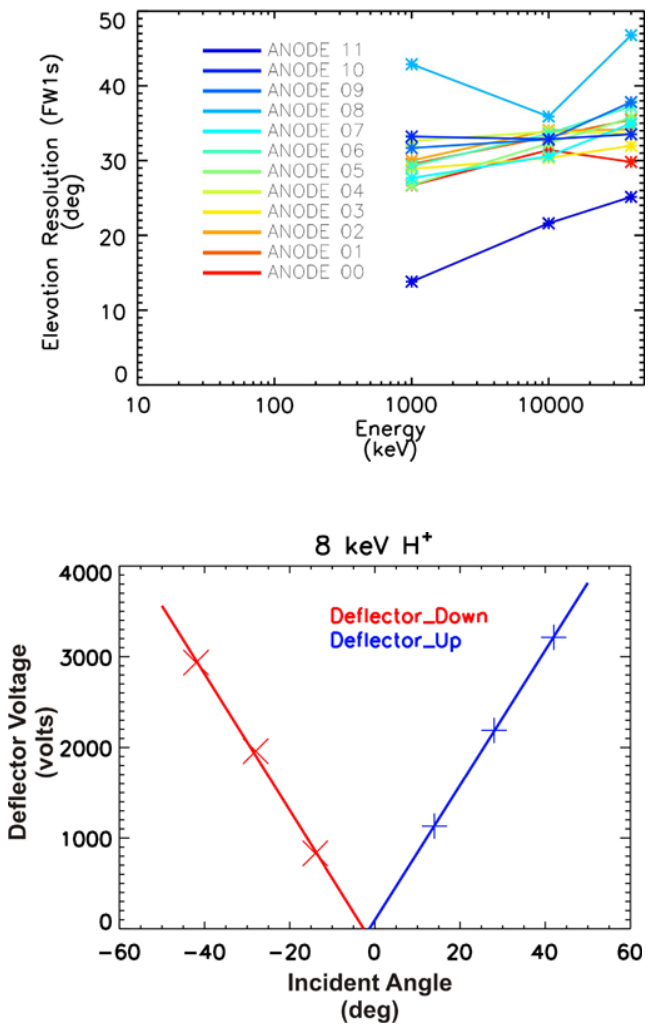

Fig. 64 Geometric factor as determined from SIMION particle ray tracing. This geometric factor assumes $100 \%$ transmission for all grids and foils, and $100 \%$ detection efficiency of the MCPs

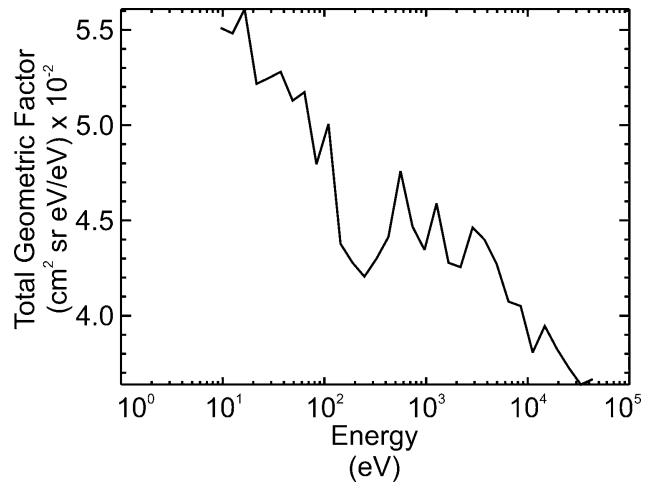

The geometric factor for JADE-I was determined through particle ray tracing based on SIMION modeling where $2.6 \times 10^{6}$ ions were launched into the JADE-I electro-optics for each of the 32 nominal ESA voltages settings; it was also measured directly in calibration using 10 and $40 \mathrm{keV}$ ions of 3 different species. Figure 64 shows geometric factor assuming $100 \%$ foil and grid transmission and detection efficiency.

During flight calibration, we measured the total effective geometric factor, including actual transmission through all grids and structures, and the measurement efficiency of the MCPs. The incident flux of particles was measured using the ABM. Figure 65 shows the 

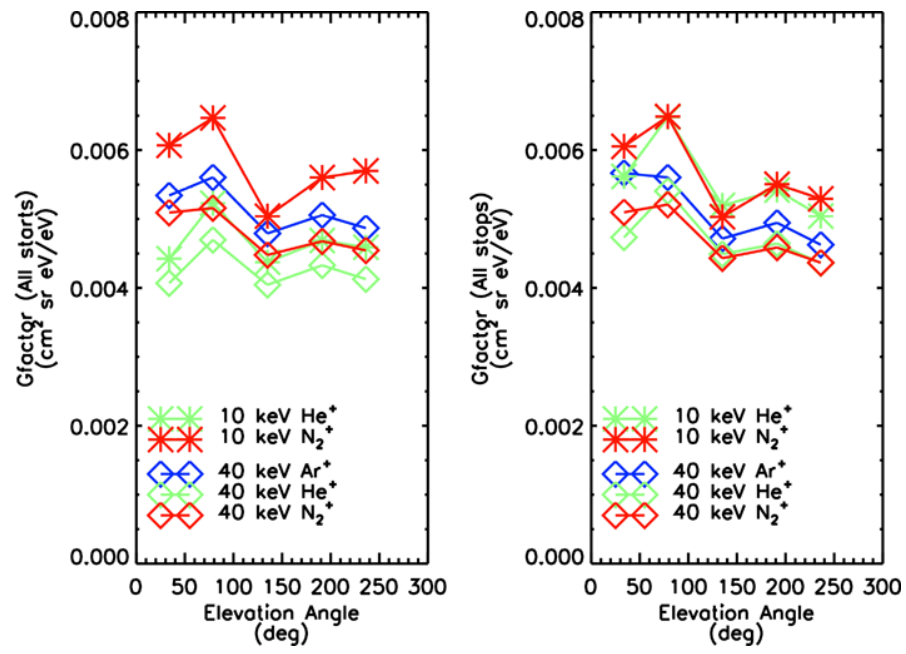

Fig. 65 Geometric factor measured during calibration includes detector efficiency. The dip seen at $135^{\circ}$ is where the ion beam is incident at an angle between two anodes and mechanical structures in the instrument partially obscure the beam

Fig. 66 Cross talk between anodes as a function of MCP voltage

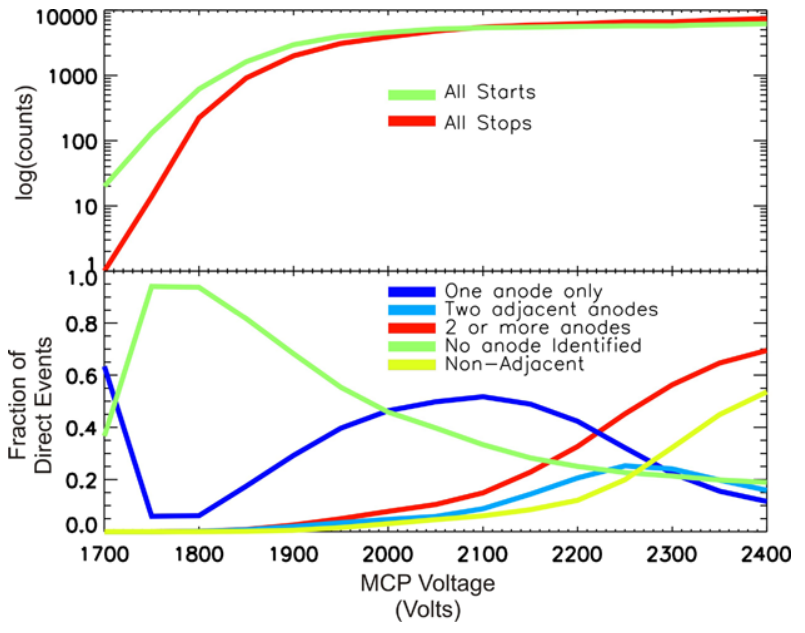

measured geometric factor for STARTS and STOPS as a function of elevation angle for three species and two energies. The sensor has a mechanical structure between each anode. The inner $=0$ data is taken when the beam is incident between two anodes and partially obstructed by these mechanical structures. The geometric factor for events with valid TOF is $\sim 4 \times 10^{-4} \mathrm{~cm}^{2} \mathrm{sr} \mathrm{eV} / \mathrm{eV}$ for the full FOV and $\sim 4 \times 10^{-5} \mathrm{~cm}^{2} \mathrm{sreV} / \mathrm{eV}$ per pixel.

The MCPs were burned in and fully characterized prior to installation in the flight sensor. In addition to the burn in process, an MCP gain curve was determined in calibration. In this test, the MCP voltage was stepped from 1700 to $2400 \mathrm{~V}$ while being illuminated with a $10 \mathrm{keV}$ proton beam. Figure 66 shows the results of this test. The top panel plots the log of All Starts (green) and All Stops (red). The MCPs start being active at $1700 \mathrm{~V}$ and are starting to reach saturation by $\sim 1900 \mathrm{~V}$. 
Fig. 67 Mass per charge response of the JADE-I TOF analyzer. Included in the data are ions with incident energies of 1 (blue), 10 (orange) and $40 \mathrm{keV}$ (red). The top panel plots the TOF vs mass per charge. The bottom panel plots the mass resolution vs mass per charge. The dashed colored lines are provided to help guide the eye. The dot-dashed line is at a mass resolution of 2.5

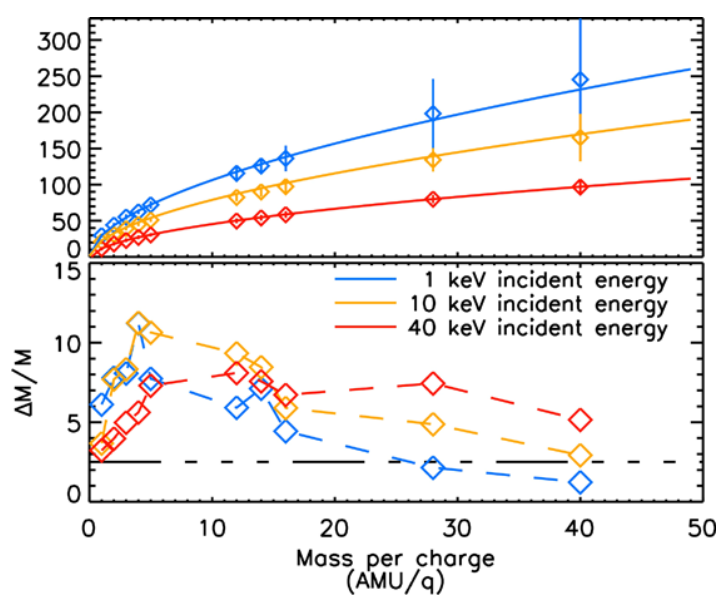

The lower panel of Fig. 66 shows how the anode imaging responds to the MCP gain. Plotted are the fraction of direct events that are seen in only one anode (dark blue), 2 adjacent anodes (light blue), non-adjacent anodes (yellow), any combination of more than one anode (red), and events where no anode could be identified (green). For cases where 2 adjacent anodes are simultaneously identified, the event is mapped to the lower anode number in addition to incrementing a counter of the number of adjacent events. Non-adjacent anode events are defined as any event that is not a single anode or 2 anodes that are not adjacent.

JADE nominal operations include periodic calibration runs where the MCPs are swept to verify that the detectors continue to operate at their proper levels. At the time of instrument turn on in space, JADE-I operated at $2100 \mathrm{~V}$, an optimal setting of the MCP gain for good anode imaging. Calibration runs are performed once every other orbit. The MCP voltages are adjusted as needed to maintain optimal performance of the sensor.

\subsubsection{Mass Analysis}

To characterize the mass resolution, the analyzer was set to measure 1,10 , and $40 \mathrm{keV} / \mathrm{q}$ ions while the calibration source mass was swept from 1 to $40 \mathrm{keV}$. The gas feed into the ion source consisted of a mixture of $\mathrm{H}, \mathrm{He}, \mathrm{N}, \mathrm{Ne}$, and Ar. Small leaks contributed additional masses of $\mathrm{C}$ and $\mathrm{O}$ plus common molecules (e.g. $\mathrm{H}_{2} \mathrm{O}, \mathrm{CO}_{2}$, etc.). The response of the sensor as a function of mass/charge is plotted in Fig. 67.

The mass per charge (Eq. (3.17)) is fit as a function of the measured TOF and the energy per charge of the ions at the carbon foil

$$
\frac{M}{q}=\left(\frac{t-C\left(E_{T}\right)}{A\left(E_{T}\right)}\right)^{1 / P\left(E_{T}\right)}
$$

where $E_{T}=$ Total energy/q at the carbon foil in $\mathrm{keV} / \mathrm{q}=$ incident energy/q $+10 \mathrm{keV} / \mathrm{q}$ and

$$
\begin{aligned}
C\left(E_{T}\right) & =1.75+2.45 \times 10^{-2} \cdot E_{T}-2.17 \times 10^{-3} \cdot E_{T}^{2} A\left(E_{T}\right) \\
& =1.78+87.8 \div \sqrt{E_{T}} P\left(E_{T}\right)=0.580-1.03 \times 10^{-3} \cdot E_{T} C\left(E_{T}\right) \\
& =1.75+2.45 \times 10^{-2} \cdot E_{T}-2.17 \times 10^{-3} \cdot E_{T}^{2}
\end{aligned}
$$




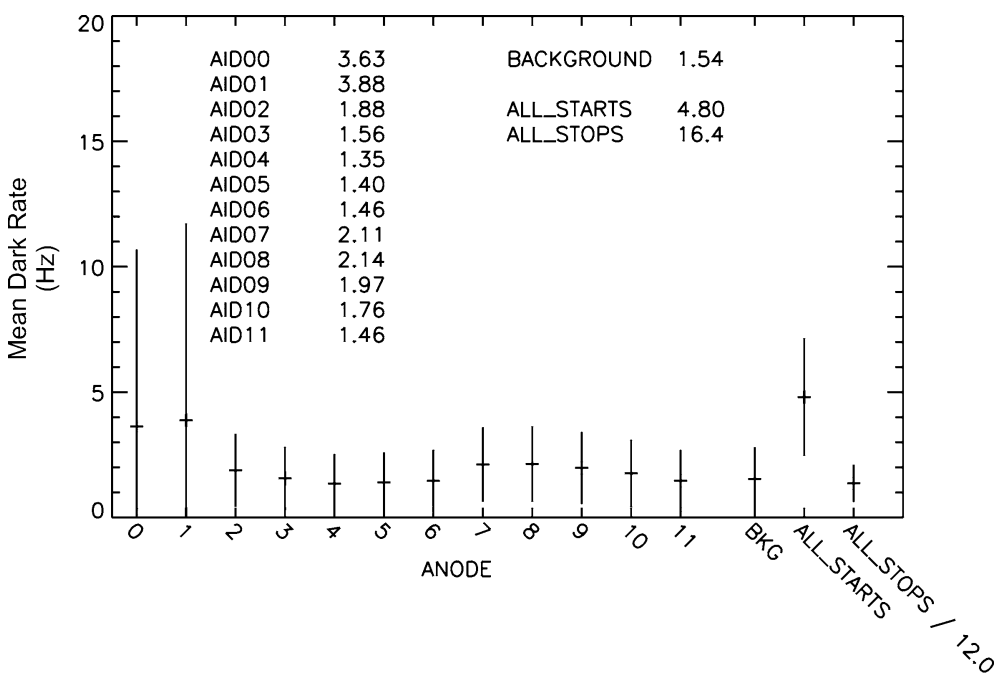

Fig. 68 Dark rate observed in the JADE-I sensor during flight calibration. The mean dark rate for each anode is indicated by the horizontal line, and the vertical line is $\pm 1 \sigma$

$$
\begin{aligned}
& A\left(E_{T}\right)=1.78+87.8 \div \sqrt{E_{T}} \\
& P\left(E_{T}\right)=0.580-1.03 \times 10^{-3} \cdot E_{T}
\end{aligned}
$$

The mass resolution, $\Delta M / M$, varies with both mass and energy. The smallest masses (i.e. protons) at the highest energies have TOFs short enough for the finite resolution of the electronics become significant. For the largest masses (>32 amu/q) at the lower energies, scattering and energy straggling in the carbon foil become the limiting factor for the mass resolution. The mass resolution was determined as the FWHM of the fitted TOF distribution at each tested mass.

\subsubsection{Upper Limit for UV Contamination}

The JADE-I sensor was operated under dark conditions for $\sim 6$ hours to measure the sensor dark rate, while the MCPs were operated at voltages slightly above the operating levels. Under these conditions, the dark rate was typically less than $1 \mathrm{~Hz} / \mathrm{cm}^{2}$ for both the start and stop anodes. The dark rates are shown in Fig. 68.

In order to determine the sensitivity of JADE-I to UV light we used a krypton lamp with $\sim 1$-sun intensity to survey the sensor with full operating voltage on the MCPs. The peak count rate seen during this test was $25 \mathrm{~Hz}$ on any stop anode, and mapping of the UV response was done for all anodes, azimuths, and elevation angles. The counts seen on the start anode are larger by a factor of $\sim 10$. While the start anode is larger than the stop anodes $\left(7.07 \mathrm{~cm}^{2}\right.$ for the start anode vs $2.79 \mathrm{~cm}^{2}$ for each stop anode), the larger number of starts from UV is probably due to photo-electrons being created inside the sensor. Since the JADE-I sensor primarily operates at the distance of Jupiter's orbit, the sensitivity to stray light in the sensor is not significant, and we estimate a background rate at Jupiter (5 AU) of $\sim 0.2 \mathrm{~Hz}$. 


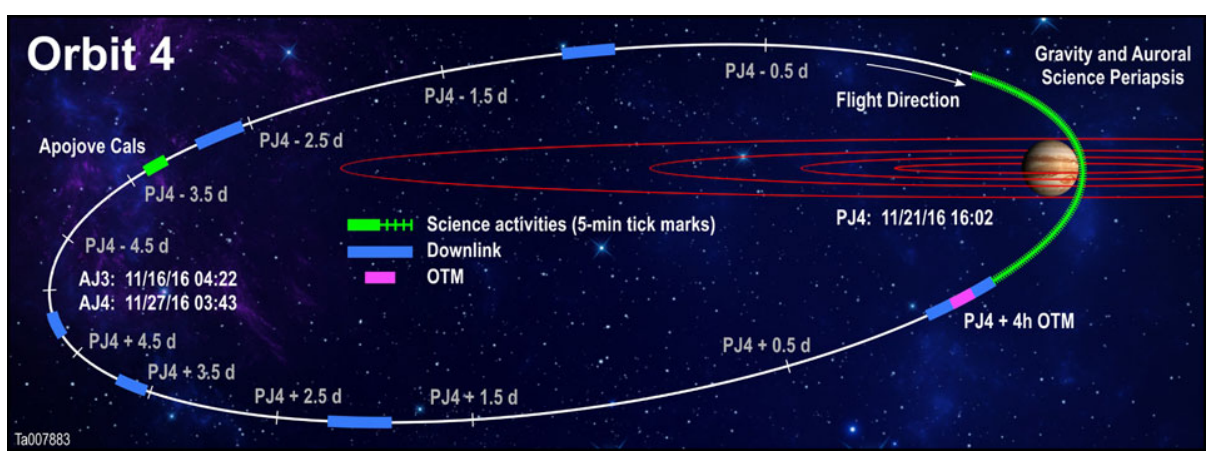

Fig. 69 JADE Science operations as a function of orbit location

\section{Science Operations, Data Processing \& Data Products}

\subsection{Science Operations}

JADE science operations are broken into orbital regions as defined by time and key locations. The orbital events that define JADE operations are perijove and the magnetic equator (current sheet) crossing around apojove. High rate science (HRS) data are taken at \pm 3 hours around perijove and Bin Recording Burst data are taken over the auroral zone (indicated by green regions in Fig. 69). The majority of the orbit is spent in LRS mode with typical accumulation times of 5 or 10 minutes, depending on available telemetry. In the transition region approaching and leaving perijove, but before HRS, we collect LRS data with an intermediate rate of 30 or $60 \mathrm{~s}$, again depending on available telemetry. This intermediate rate LRS will also be used near the equatorial current sheet around apojove.

The Juno attitude difference during MWR and GRAV orbits (see Fig. 70), affects the quality of JADE electron data. In general, a non-deflected data set is more accurate than a deflected one, and due to observational geometry, even a perfectly deflected beam is not equal to a non-deflected beam. Because of this, orbits where the JADE FOV is more field aligned in the auroral regions, such as most early MWR orbits, provide better science collection because they require less deflection.

\subsubsection{Periapsis}

JADE primary science is collected \pm 3 hours around perijove, where the spacecraft passes through the auroral region in the northern hemisphere, the Jovian equatorial plane and the auroral region in the southern hemisphere. Due to both the variability associated with the auroral regions on short time scales, and the fast spacecraft motion, high time resolution data is needed.

Three hours before perijove JADE enters HRS, with all sensors collecting data, JADE-I sweeping ESA and DFL by table, JADE-E sweeping ESA by table, and deflectors set to remain field aligned based on the real time MAG data. During this time high-fidelity histograms and direct events (as bandwidth allows) are collected (as described in Sect. 4.2). While JADE passes through the northern auroral region, Burst is enabled. The location of the auroral crossing changes slightly with each orbit, thus the amount of time Burst is enabled and the timing relative to perijove changes each orbit (see Bagenal et al. 2013, this issue). Each auroral crossing captures approximately seven 60-second Waves-triggered Burst 
Fig. 70 Juno attitude for radiometer (MWR) and gravity (GRAV) orbits

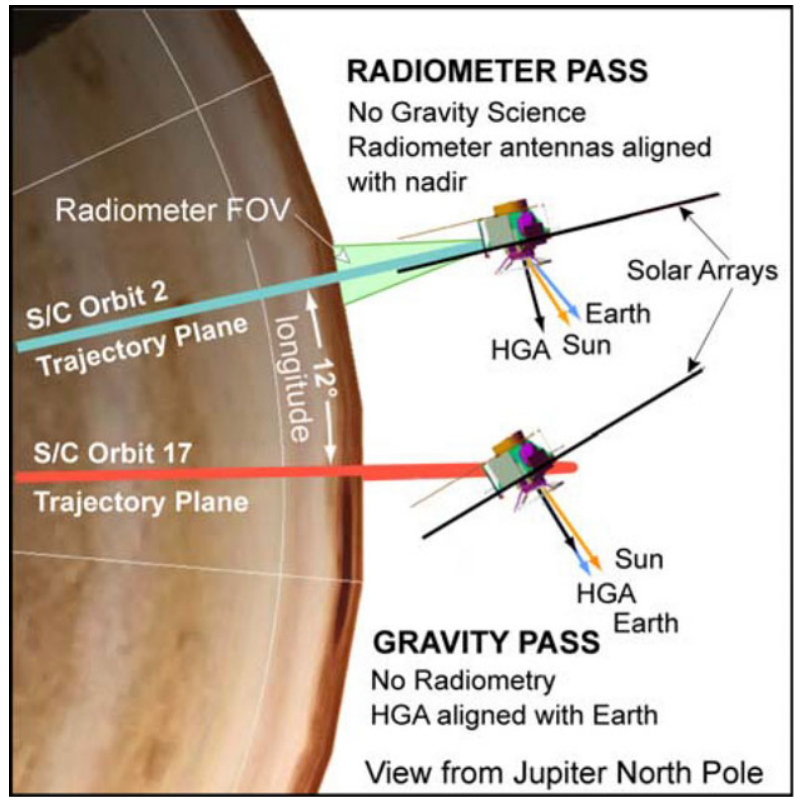

intervals, as described below. Between auroral crossings JADE is in HRS collecting high fidelity histograms and direct events (as bandwidth allows) across the equatorial region. At the southern auroral zone JADE is again put into Burst mode. Because of the orbit evolution, the spacecraft altitude is lower and southern auroral mapping is better earlier in the mission.

4.1.1.1 Burst During Auroral Field Line Crossings The most critical JADE measurements occur on the auroral field-line crossings. Active field lines are small-less than 1,000 km wide according to HST observations. Juno crosses these field lines at $\sim 50 \mathrm{~km} / \mathrm{s}$, and the critical data occur in $\sim 20$ s intervals, with the exact timing of the crossings unknown.

Burst mode is designed to capture these auroral crossings. The highest possible resolution data is captured and prioritized by how 'interesting' the data is, as defined by the Waves quality factor (see Sect. 4.4.2.6 for Burst details). Waves flight software sets the quality flag based on (1) the number of solitary structures in the Waves wideband data, (2) the amplitude of waves near the electron cyclotron frequency, and (3) sudden changes in broadcast magnetic field direction or magnitude (Kurth et al. 2013, this issue). Waves broadcasts a Burst trigger when the quality flag rating is above a threshold. When a Waves trigger is broadcast, the spacecraft continues collecting data for $30 \mathrm{~s}$; data $\pm 30 \mathrm{~s}$ from the trigger are stored with the associated quality flag. There is telemetry to transmit $\sim 760$-s bursts during each perijove, and more are added to the plan as bandwidth allows.

JADE can only store burst data if it is in Burst mode when the Waves trigger is sent. Burst mode recording sessions are defined by time-tagged command loads uploaded 2 orbits in advance of execution. These recording sessions are planned during the auroral crossings using trajectory and field-line model predictions.

\subsubsection{Intermediate Accumulation LRS Near Perijove}

It is preferred to have a ramp-up of data capture both just before and just after HRS around perijove. Therefore, JADE has an intermediate rate LRS period in these transition regions 
where the histogram accumulation rate is increased to 30 or $60 \mathrm{~s}$, depending on availability of telemetry. This allows JADE to acquire higher time resolution data over regions of interest flanking the Juno-defined perijove regions.

\subsubsection{Orbital Trim Maneuver (OTM)}

Each orbit an OTM is needed to precisely time the orbits with respect to Jupiter's rotation rate so that evenly spaced global maps of Jupiter can be gathered. These OTMs are scheduled for 4 hours after each perijove, although the precise timing may evolve. Because of the use of the Juno thrusters for the OTM, JADE pauses data collection and JADE-I MCP and TOF are set to $0 \mathrm{~V}$. The spacecraft mini-sequence that commands OTMs also contains commands to ramp the noted JADE voltages down prior to the maneuver, and then ramp them back up after the maneuver. Once the voltages are restored to nominal operating voltage, data collection resumes in LRS.

\subsubsection{Low Rate Science (LRS) Accumulation During Bulk of Orbit}

After the perijove region (and any intermediate accumulation LRS following it), JADE shifts to collecting data in low accumulation rate, for the bulk of the orbit since high time resolution is not needed away further from Jupiter in the more distant magnetosphere. In LRS, all electron and species data are binned by spin phase and only histogram data are stored. ESA sweeping is performed for both sensors, and DFL sweeping via table is performed for JADE-E. Essentially $4 \pi$ viewing is available for JADE-I due to the spacecraft spin.

\subsubsection{MCP Calibration During Current Sheet Crossing}

JADE regularly (typically once every other orbit) enters MCP Calibration mode as it crosses the current sheet near apojove. Once in this mode a macro is run to perform a standard gain test on the MCPs, in order to ensure that the operating voltages are on their gain curve plateaus. As a part of normal detector degradation, the operating voltages on the MCPs may need to be increased over the mission life to maintain high count efficiency. If and when an operating voltage needs to be changed, new macros are uploaded to incorporate the new values.

\subsubsection{Intermediate Accumulation LRS During Current Sheet Crossing}

If bandwidth allows, JADE increases accumulation rates to intermediate levels while passing through the current sheet near apojove. This is performed for no less than 12 hours to allow separation of temporal and spatial signatures in the data, therefore the collection period must be longer than the Jovian rotation rate of $\sim 10$ hours.

\subsubsection{Other Science}

4.1.7.1 Post-Perihelion Initial Ramp Up JADE-I temperatures were $\sim 20^{\circ}$ above those predicted by the pre-launch thermal model. JADE-I neared, but did not exceed, non-operational limits shortly after launch and fell to acceptable values for the initial high voltage turn on. Initial science observations from that turn-on are shown in Sect. 5. After perihelion, when JADE temperatures fall below post-perihelion operational limits, the instrument will be turned on again with additional precautions. 
4.1.7.2 Periodic Instrument Maintenance Juno has 3 designated week-long periods for Periodic Instrument Maintenance (PIM). JADE will not participate in Earth flyby or the first PIM due to thermal constraints. However, JADE will participate in the second PIM in 2014 and the final PIM in 2015. During these periodic maintenances, JADE starts with a gain test early in the activity, spends time in each mode including coordinated Burst mode periods with Waves, and concludes with a gain curve at the end of the activity.

4.1.7.3 Cruise Science When bandwidth allows, and additional science can be obtained without increasing mission risk or compromising long-term objectives, JADE participates in cruise science activities using LRS mode. If bandwidth allows the standard $600 \mathrm{~s}$ accumulation rate will be decreased to 300,60 or $30 \mathrm{~s}$ and an additional ion species may also be transmitted. Cruise science activities are conducted using standard command packages to turn on, set the initial configuration, and then dwell in that mode. Minimal changes are made to keep operations and bookkeeping as simple as possible.

4.1.7.4 Approach Science JADE participates in approach science activities as bandwidth and power allow, on a non-interference basis with primary mission objectives. Approach science consists primarily of LRS, which can be augmented with additional activities based on consensus among the MWG and project.

4.1.7.5 Capture Orbit JADE takes LRS data during the capture orbit, as bandwidth and power allow, on a non-interference basis with primary mission objectives. JADE is planned to operate until just a few days before JOI and then turn off for JOI. JADE will turn back on as soon after JOI as possible and resume LRS operations. A model prediction shows tens of magnetosheath crossings within the capture orbit (Bagenal et al. 2013, this issue), providing intriguing secondary science opportunities. Additionally, JADE will work with the MWG on any coordinated instrument efforts during the capture orbit.

\subsection{Energy and Angle Scanning Strategy}

\subsubsection{Data Resolution by Mode}

The JADE data resolution for each mode is described in this section and summarized in Table 16.

4.2.1.1 JADE-E Data Resolution by Mode JADE-E has an inherent resolution of 64 energies $\times 48$ directions $\times 1 \mathrm{~s}$. Sweeping and data collapsing are dependent on mode.

In HRS and Burst the JSIB sweeps all 64 ESA/DFL pairs in $1 \mathrm{~s}$. In Burst there is no collapse of energy, direction or time resolution. In HRS the data is collapsed to 32 energies $\times$ 24 directions $\times 1 \mathrm{~s}$ resolution, with directions collapsed according to pitch angle. Pitch angles within $0-60^{\circ}$, and $120-180^{\circ}$ from the magnetic field direction are uncollapsed in direction. The remaining bins are collapsed from 4 adjacent fine-pitch angle bins to 1 course pitch-angle bin as shown in Fig. 71.

In LRS and Cal, the data is not collapsed, and retains 64 energies. There is no directional energy collapse based on pitch angle because all data are taken in a plane. The deflectors are swept, but not to magnetic field alignment. Rather than $1 \mathrm{~s}$ resolution, the data are binned into histograms with typically 5 or 10 minute resolution for Low Rate LRS, and 30 or $60 \mathrm{~s}$ for Intermediate Rate LRS and Cal. 
Table 16 Summary of JADE resolution by mode. PA = pitch angle; En = energy; El = Elevation; Dir = direction; $\mathrm{Az}=$ Azimuth; $\mathrm{TOF}=$ Time of Flight; $\mathrm{sp}=$ ion species

\begin{tabular}{|c|c|c|c|c|c|}
\hline & Data type & Resolution & $\begin{array}{l}\text { Cadence } \\
\text { (seconds) }\end{array}$ & $\begin{array}{l}\text { Rate } \\
\text { [bps] }\end{array}$ & $\begin{array}{l}\text { Total rate } \\
\text { [bps] }\end{array}$ \\
\hline \multirow[t]{3}{*}{ BURST } & JADE-E & $64 \mathrm{En} \times 48 \mathrm{PA}$ & 1 & 24576 & \multirow[t]{3}{*}{69632} \\
\hline & JADE-I spectra & $32 \mathrm{En} \times 8 \mathrm{El} \times 12 \mathrm{Az} \times 6 \mathrm{sp}$ & 4 & 36864 & \\
\hline & JADE-I TOF & $32 \mathrm{En} \times 128 \mathrm{TOF}$ & 4 & 8192 & \\
\hline \multirow[t]{3}{*}{ HRS } & JADE-E & $32 \mathrm{En} \times 24 \mathrm{PA}$ & 1 & 6144 & \multirow[t]{3}{*}{16384} \\
\hline & JADE-I spectra & $32 \mathrm{En} \times 4 \mathrm{El} \times 12 \mathrm{Az} \times 2 \mathrm{sp}$ & 4 & 6144 & \\
\hline & JADE-I TOF & $16 \mathrm{En} \times 128 \mathrm{TOF}$ & 4 & 4096 & \\
\hline \multirow[t]{3}{*}{ LRS } & JADE-E & $64 \mathrm{En} \times 24 \mathrm{PA}$ & 30 or 300 & 20.48 & \multirow[t]{3}{*}{58.1} \\
\hline & JADE-I spectra & $32 \mathrm{En} \times 56 \operatorname{dir} \times 1 \mathrm{sp}$ & 30 or 300 & 23.9 & \\
\hline & JADE-I TOF & $16 \mathrm{En} \times 64 \mathrm{TOF}$ & 30 or 300 & 13.7 & \\
\hline \multirow[t]{3}{*}{$\mathrm{MCP} \mathrm{Cal}$} & JADE-E & $16 \mathrm{PA} \times 64 \mathrm{En}$ & 30 & 819.2 & \multirow[t]{3}{*}{856.8} \\
\hline & JADE-I spectra & $32 \mathrm{En} \times 56 \mathrm{dir} \times 1 \mathrm{sp}$ & 30 & 23.9 & \\
\hline & JADE-I TOF & $16 \mathrm{En} \times 64 \mathrm{TOF}$ & 30 & 13.7 & \\
\hline
\end{tabular}

Note: JADE-I can also transmit optional species (not included)

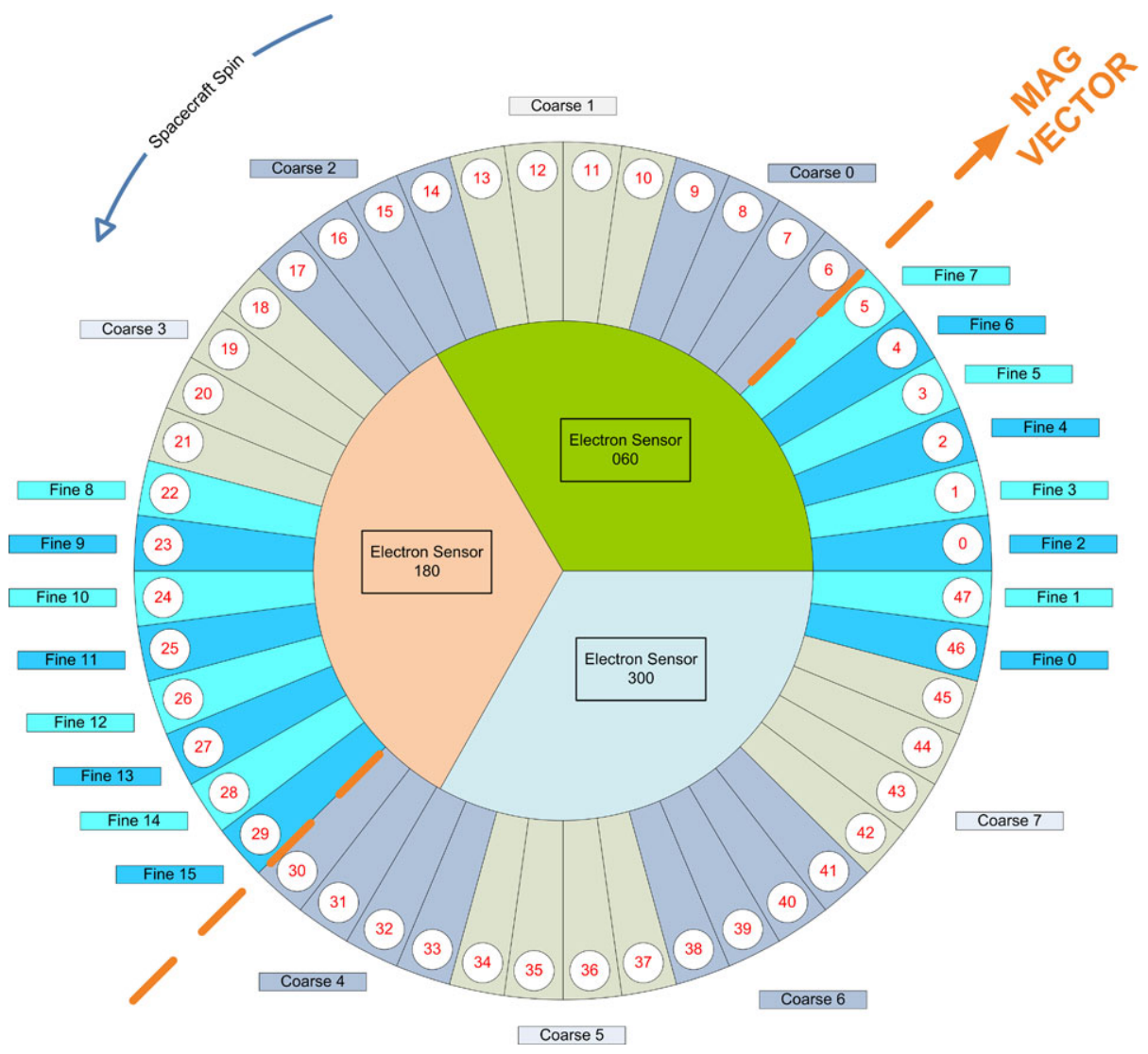

Fig. 71 JADE-E data collapse based on broadcast magnetic field data 


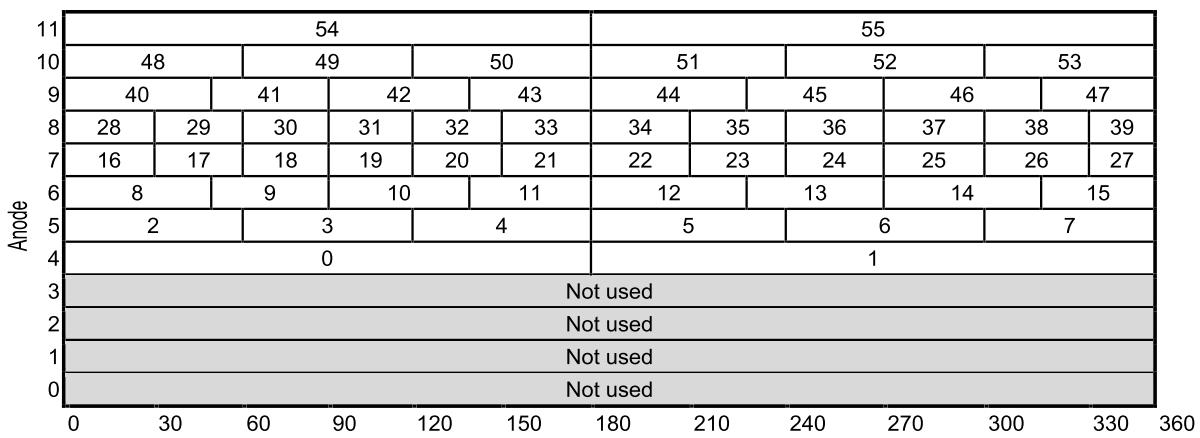

Fig. 72 JADE-I anode mapping by spin phase $\left(0-360^{\circ}\right)$ in LRS

4.2.1.2 JADE-I Ion Data Resolution by Mode Sweeping, species, and data collapsing are dependent on mode. In HRS and Burst modes, JADE-I's inherent resolution is 32 energies $\times$ 8 elevations $\times 12$ azimuths $\times 256 \mathrm{TOF} \times 4 \mathrm{~s}$. Based on LUTs, ranges of TOF for each energy step are defined as species. Species 0 and 1 are predefined as being any events measured on any stop anode and any event with a valid TOF, respectively. Species 2 is for mass per charge of $1 \mathrm{amu} / \mathrm{q}$, species 3 is for light components of the plasma (mass per charge between 2 and $5 \mathrm{amu} / \mathrm{q}$ ), and species 4 is for heavy components of the plasma (mass per charge $>5 \mathrm{amu} / \mathrm{q}$ ). Up to 3 more species can be identified, and the values for species 2-7 can be redefined by upload of a table at any time.

In Burst there is no data collapse, and up to 6 species are stored configurable by command. In HRS the data is only collapsed in elevation by summing adjacent elevations; the data stored is 32 energies $\times 4$ elevations $\times 12$ azimuths for each transmitted species (default 2 species in HRS, configurable by command). An additional 'optional' species can be transmitted while in HRS. Our default configuration will be to transmit species 2 and 4, and have species 3 as the optional species while in HRS.

In LRS and Cal, the data is 32 energies $\times 56$ directions $\times 1$ species. Rather than $4 \mathrm{~s}$ resolution, the data are binned into histograms with 5 or 10 minute resolution for Low Rate LRS and Cal, and 30 or $60 \mathrm{~s}$ for Intermediate Rate LRS. JADE calculates the spin phase at each observation cycle boundary based on the time stamp of the spin phase provided by the spacecraft. JADE-I bins translate to 56 near-constant solid angle sectors. In LRS the data is mapped per spin, such that the counts in each anode are mapped over approximately equal solid angles. Since anodes 4 and 11 look nearly along the spin axis, in half a spin they sweep out about the same amount of solid angle as anodes 7 and 8 sweep out in about $15^{\circ}$ of rotation, therefore in LRS, the count rates for each anode are mapped to different sized bins in spin phase (see Fig. 72). Anodes 0-3 are not used in LRS for the species data since they view the same region of space as anodes $4-7$ out of phase by $180^{\circ}$.

4.2.1.3 JADE-I TOF Resolution by Mode JADE-I inherent TOF resolution is 32 energies $\times$ $256 \mathrm{TOF} \times 1 \mathrm{~s}$ over all available directions. In Burst science the TOF data is collapsed such that elements $0-83$ are mapped one to one, and for TOF bins 84-255 the 4 consecutive bins are collapsed (i.e. bins $84,85,86$, and 87 are collapsed and mapped to 84). In HRS, adjacent energies are collapsed to provide a resolution of 16 energies $\times 128 \mathrm{TOF} \times 4 \mathrm{~s}$ resolution. The same TOF collapsing as in burst is performed in HRS. In LRS and Cal, TOF data is collapsed into 16 energies $\times 64$ TOFs. In all modes, the TOF data is summed over all elevations and azimuths covered in the accumulation time. 
Fig. 73 One JADE-E ESA sweep

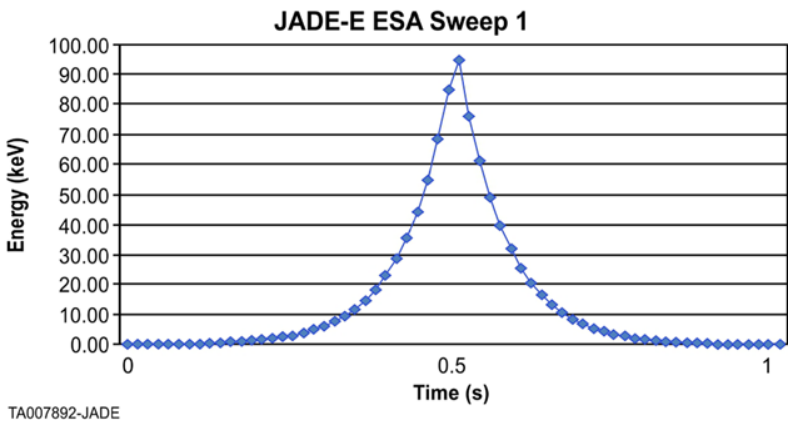

\subsubsection{Energy Sweeping by Mode}

4.2.2.1 JADE-E Sweeping by Mode JADE-E sweeps through its 64 ESA steps in $1 \mathrm{~s}$, regardless of mode. A low-resolution, 32-step sweep is taken every $0.5 \mathrm{~s}$, hitting every other energy step in the first half second where voltage is increasing, then filling in the skipped voltages during the second half of the second where the voltages are decreasing. ESA steps are $0,2,4, \ldots, 30,31,29, \ldots, 1$ (Fig. 73). The reason for sweeping up then down is to minimize the largest single step taken and therefore minimizing the HVPS settling time. Because the intrinsic resolution of JADE data is $1 \mathrm{~s}$, no additional temporal resolution is required.

There are 3 JADE-E sweep tables that can be updated; at launch, table 1 covered the full energy range from 0.1 to $\sim 100 \mathrm{keV}$, table 2 is a limited range solar wind sweep from $0.1-5 \mathrm{keV}$, and table 3 is a calibration table with 4 energies.

In HRS and Burst all 3 JADE-E sensors collect data, and deflectors are set to remain field-aligned according to the real-time magnetic field. The JADE software compensates for an $\sim 1 \mathrm{~s}$ latency in this vector by propagating from its last position to the time at the next sweep start, using the updated spin rate. In LRS only one sensor is operational. In Cal, all three sensors are active. Deflection is based on sweep tables, not magnetic field alignment.

4.2.2.2 JADE-I Sweeping by Mode JADE-I makes use of low-resolution scans within the full ESA range to allow for greater temporal resolution over a large energy range. JADE-I has 32 ESA steps, but how long it remains in each step and how the voltages are ramped depend on the mode.

In HRS, one 32-step high-resolution ESA sweep is completed every $4 \mathrm{~s}$, while each 8-step low-resolution ESA sweep is completed every $1 \mathrm{~s}$. The low-resolution scans allow for greater temporal resolution than a sequential scan. ESA steps are $0,4,8,12,16,20,24,28,29,25$, $21,17,13,9,5,1,2,6,10,14,18,22,26,30,31,27,23,19,15,11,7,3$ as shown in Fig. 74.

In HRS, the deflector is scanned 8 DFL steps for each ESA step (covering $\pm 45^{\circ}$ deflection), covering $64 \mathrm{ESA} / \mathrm{DFL}$ pairs per second. There are two sweep tables available for use in HRS that can be updated anytime by uploading new tables. The tables at launch are set to cover the full energy range. The first HRS sweep table sweeps the deflectors over 8 steps, spaced $11.25^{\circ}$ apart, while the second sweeps the deflectors over 8 steps, spaced $6^{\circ}$ apart.

In LRS and Cal, the ESA supply sweeps according to one of four sweep tables that command 32 voltage steps per second. The tables can be updated at anytime, with tables loaded at launch having 32 logarithmically spaced steps arranged in a pyramid sweep such that even numbered voltage steps ramp up in the first half second and the odd numbered voltage steps ramp down in the second half second (Fig. 75).

There are 4 sweep tables available in LRS that can also be updated. At launch, the first table covered the energy rang of $10 \mathrm{eV}$ to $45 \mathrm{keV}$, and is the default table to be used at 
Fig. 74 One JADE-I HRS ESA sweep

Fig. 75 One JADE-I LRS/Cal ESA sweep

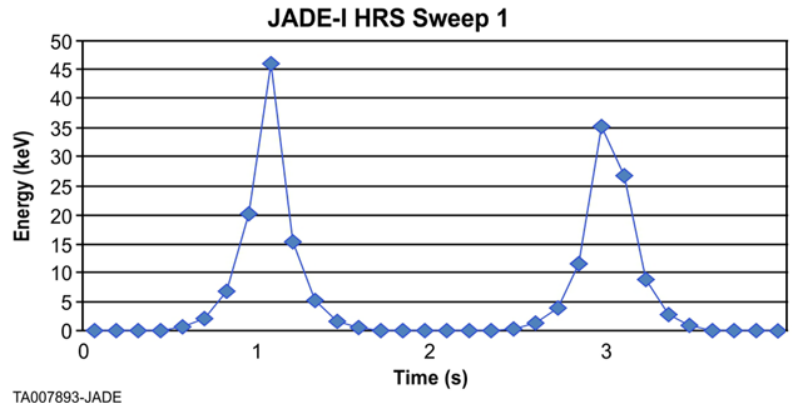

TA007893-JADE

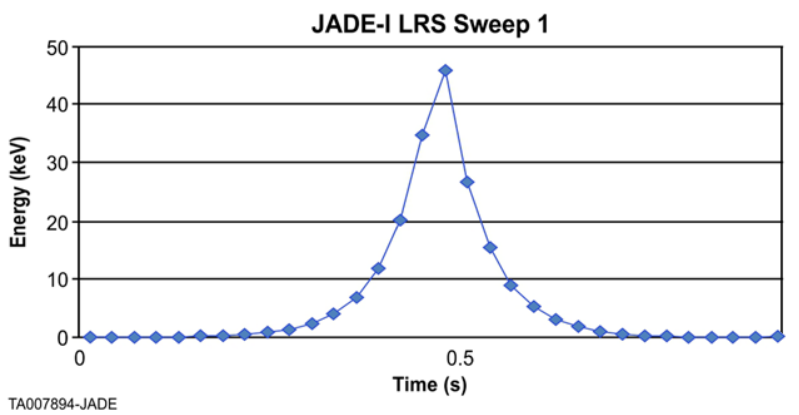

Jupiter, while the second covers the energy range from $350 \mathrm{eV}$ to $7.7 \mathrm{keV}$ to be used during cruise to measure the solar wind; this energy range will cover the most probable solar wind velocities (Gosling 2007). The third table covers energies from $125 \mathrm{eV}$ to $45 \mathrm{keV}$, and the fourth, energies from $5 \mathrm{eV}$ to $25 \mathrm{keV}$. The deflector supplies do not sweep in this mode.

4.2.2.3. Histogram Binning All histogram data is organized by spin phase. JADE calculates the spin phase at each observation cycle boundary based on the time stamp of the spin phase provided by the spacecraft. JADE-I bins translate to 56 near-constant solid angle sectors. In LRS the data is mapped per spin, such that the counts in each anode are mapped over approximately equal solid angles. Since anodes 4 and 11 look nearly along the spin axis, in half a spin they sweep out about the same amount of solid angle as anodes 7 and 8 sweep out in about $15^{\circ}$ of rotation, therefore in LRS, the count rates for each anode are mapped to different sized bins in spin phase (see Fig. 72).

The JADE-E lookup tables controlling the ESA and DFL voltages in LRS are fixed. These tables contain 64 steps of ESA and DFL voltage combinations. For example, one can chose a table with 64 energies with zero voltage on the DFL. Alternately, one can chose a different table with 16 ESA voltages (e.g. 16 energies) and 4 DFL voltages for each energy step. The voltage sweep is different, but the total of ESA-DFL voltage combinations is the same. The LRS data products for JADE-E are independent of the lookup table and are always binned in an array of counts with dimension $64 \times 24$. We reconstruct the count rate versus energy by look direction using the lookup tables with ground processing.

4.2.2.4. Determining Count Rate The ion species and electron products in LRS are in units of rates (counts per views) instead of counts. If a sector is viewed 2 times and has 1 count, the rate is 0.5 , which is saved as 0 (losing data with integer truncation). So, rates are multiplied by 512 before being sent to the ground. Then the ground software then divides the rates by 512 into floating point numbers, and preserves decimal rates. 


\subsection{Operation and Processing by JSIB and Flight Software}

The following sections describe which JADE functions are controlled directly by the JSIB and which are controlled by the Flight Software (FSW).

\subsubsection{Electron Sensor Switched Power}

The JSIB controls the power to the in-sensor processing electronics of the three JADE-Es. The power is individually switchable for each sensor. FSW controls which electron sensors are powered by setting power control bits in JSIB FPGA registers. FSW is commanded to enable/disable electron sensors based on the mode to conserve power. Commands nominally come from in-JADE stored sequences (macros).

\subsubsection{High Voltage Control}

Each high voltage power supply can also be individually switched on/off by the JSIB. FSW controls which supplies are enabled for each mode by setting power control bits in JSIB FPGA registers. FSW is commanded to enable/disable high voltage sources based on the mode to conserve power. Commands nominally come from onboard via stored sequences or macros. The ion sensor and electron sensors each have an MCP, an ESA, and two DFL voltages. Additionally, the ion sensor has a TOF voltage. Since JADE-E's ESAs and deflectors both have positive polarity they use a single bulk power supply. However, because JADEI's ESA uses a negative voltage and the deflectors have a positive voltage, JADE requires separate bulk power supplies for the positive and negative voltages.

The JADE-I MCP and TOF are static supplies, staying at a fixed voltage during science data collection after being commanded to the appropriate voltage. FSW is commanded to the appropriate MCP and TOF voltages through macros and writes 12-bit raw digital values to the JSIB FPGA control registers.

The ESA and DFL are stepper supplies, designed to rapidly sweep through a range of voltages. The FSW retrieves voltage sweep tables stored in non-volatile memory (EEPROM), and writes these 13-bit raw digital values to arrays mapped into the JSIB FPGA memory. The sweep tables are selectable via command. The ESA and DFL are dual-range supplies. The 13th bit in each value is the range bit, selecting whether the 12-bit raw value should be mapped to hundreds of volts or thousands of volts. The FSW only calculates sweep tables on-the-fly during HRS and Burst science, and only for the electron sensor deflection; the electron sensors must deflect to view the magnetic field vector, which is not known in advance and must be retrieved on-board from the FGM (flux-gate magnetometer) instrument.

The JSIB controls the high voltage by using a digital to analog converter to convert the 12-bit raw voltage values into a scaled voltage interpreted by the high voltage control board. In LRS and MCP Cal, the JSIB sweeps the electron sensors with 64 ESA/DFL pairs in $1 \mathrm{~s}$, and sweeps the ion sensor with 32 ESA steps (no deflection) in $1 \mathrm{~s}$. In HRS and Burst science, the JSIB sweeps the electron sensors with 64 ESA/DFL pairs in $1 \mathrm{~s}$, and sweeps the ion sensor every $4 \mathrm{~s}$ with 32 ESA steps each containing 8 DFL steps.

\subsubsection{Analog Monitoring}

The JSIB monitors voltages, currents, and temperatures from all parts of JADE, including high voltage, low voltage, sensors, and control boards (JSIB, IPB, HVPS, and LVPS). The 
JSIB uses an analog-to-digital converter to read the scaled monitor values as 12-bit digital values. The JSIB updates all 80 housekeeping monitors every 5.12 milliseconds, and arranges the values in an array in memory, where the FSW can read it 8 times per second. The FSW stores minimum, maximum, and average values for these monitored values and checks the values against red alarm minimum and maximum values, taking action (requesting a turnoff from the spacecraft) when monitors exceed limits for a persistent (selectable) number of samples. The software also performs rate limiting and auto safing, described below in Sects. 4.4.6 and 4.4.7.

\subsubsection{Electron Sensor Data Products}

The JSIB counts the number of electron detections in each ESA/DFL step for each sensor anode. As described above in Sect. 2.4.4.3, each electron sensor has 16 positional anodes covering a $120^{\circ} \mathrm{FOV}$. Together, the three electron sensors ring the spacecraft to create a $360^{\circ}$ FOV. Additionally, each electron sensor has a background anode to count penetrating radiation. The JSIB forms a matrix in FPGA memory representing the electron sensors as a two-dimensional array, with the ESA/DFL steps as the rows, and the 51 anodes (16 . 3 positional +3 background) as the columns. This data produces a histogram of counts.

In Burst science, the FSW transmits the raw histogram from the JSIB each second as the data product, providing JADE's highest rate science data for selected intervals in the auroral zones. Owing to telemetry limitations, this product can only be provided for a small fraction of the time.

In HRS, which is available throughout the entire perijove intervals, including all auroral zone crossings, the FSW reduces the data product size by summing adjacent rows into 32 ESA/DFL steps, and prioritizing resolution in the positional anodes. The 8 anodes closest to being parallel and anti-parallel to the magnetic field vector are preserved as individual bins, creating high resolution for particle pitch angles around the field vector. The anodes furthest from the magnetic field vector are summed with 4 anodes into coarse bins (see Fig. 71). Over a spacecraft rotation, the magnetic field vector traverses around all three electron sensors, and all anodes fall into high-resolution (fine) bins. From this, a complete $360^{\circ}$ map can be created by combining the high-resolution histogram pieces on the ground. The FSW collapses each background count into a single counter, preserving total counts. The FSW produces this data product each second.

During LRS, over the rest of Juno's orbit away from the polar regions, the FSW reads the histograms from the JSIB each second and further accumulates them over a commendable amount of time depending on telemetry allocation. For most of the mission this resolution is nominally 10 minutes. This accumulation interval must be an even multiple of spacecraft spins (2 rpm).

The spin dependency is a result of the data product binning in the FSW by spin sectors. Each positional anode is mapped into a particular solid angle sector based on the orientation of the spacecraft. As the spacecraft rotates, different positional anodes fall into that sector. The FSW creates a histogram of count rates for 24 spin sectors, preserving the 64 ESA/DFL step resolution. The background count is summed into a single counter, independent of energy step. The units of count rates are created by dividing the total counts by the number of times each sector was viewed.

In MCP Calibration mode, the FSW creates a separate data product for each electron sensor, preserving the complete 64 ESA/DFL steps and 17 anodes (16 positional and 1 background). The FSW reads this histogram from the JSIB each second and accumulates it over a commendable time period (nominally 30 -s integrations). 


\subsubsection{Ion Sensor Data Products}

The ion sensor contains 12 position anodes, and a background anode for penetrating radiation. JADE-I also measures the TOF for each particle. This measurement begins when the JSIB receives a start pulse, and ends either with reception of a stop pulse from an anode, or a timeout with no stop pulse. During the measurement interval, new start pulses are locked out. The JSIB stores data products for these particles at 1-s intervals in LRS and Cal, and at 4-s intervals in HRS and Burst, which have a longer sweep time to accommodate elevation deflection not used in low rate scans.

The JSIB represents each particle as a direct event, which contains the ESA/DFL step, the TOF, anode, and quality information. A certain number of direct events are stored in a two-dimensional buffer (ESA step by event) in LRS and Cal, and three-dimensional buffer (ESA step by DFL step by event) in HRS and Burst. The FSW reads the direct events from the JSIB as an array mapped into JSIB FPGA memory and transmits them over a selectable interval (nominally 10 minutes in HRS and Burst, and 10 hours in LRS).

The JSIB histograms the TOF measurements into a data product with 32 ESA steps (all 8 DFL summed together in HRS and Burst) and 128 TOF bins (256 for Direct Event data only). The TOF information is represented in digital values corresponding to asserted delay line taps and coarse ticks, which the ground processing converts into nanoseconds. The FSW transmits this data product in Burst mode, and reduces resolution in HRS mode by summing adjacent energy rows into 16 ESA steps, preserving the 128-bin TOF resolution. In LRS and Cal modes, the FSW accumulates this product over a selectable number of seconds (nominally 5 or 10 minutes for LRS, and $30 \mathrm{~s}$ for Cal), and reduces row and column resolution by summing adjacent rows and columns to form a 16 ESA by 64 TOF histogram.

The JSIB classifies each particle with a valid TOF as a specific ion species via a lookup table provided by FSW. The FSW generates this table on-the-fly by retrieving the ESA voltage and comparing it against a stored table in non-volatile memory (EEPROM) to determine which TOF ranges map to which species for each ESA step. The FSW writes this table to the JSIB as an array in the JSIB FPGA memory space, so the JSIB can use the energy step and TOF as a lookup table to determine the correct species. The JSIB produces a data product with 32 ESA steps by 8 DFL steps by 12 positional anodes. The FSW produces this data product in Burst mode. In HRS mode, the FSW reduces DFL resolution by summing adjacent DFL steps to create a data product of 32 ESA steps by 4 DFL steps by 12 positional anodes. During LRS and Cal modes, away from the polar regions and away from the prime science, the FSW reads the histograms from the JSIB and further accumulates them over a commendable number of seconds, nominally 10 minutes in LRS and $30 \mathrm{~s}$ during Cal. This accumulation is an even multiple of spacecraft spins (30-s spin at $2 \mathrm{rpm}$ ). The reason for the spin dependency is because the data product is binned in FSW by spin sectors. Each positional anode is mapped into a solid angle sector, based on the orientation of the spacecraft. As the spacecraft rotates, different positional anodes will fall into that sector. The FSW creates a histogram of count rates for 56 spin sectors, preserving the 32 ESA step resolution. As for JADE-E, the JADE-I units of count rates are created by dividing the total counts by the number of times each sector was viewed.

The JSIB creates a singles/logicals histogram with 32 ESA steps and 25 counters in LRS and Cal, and 32 ESA steps by 8 DFL steps by 25 counters in High Rate and Burst. The 25 counters represent the 12 positional anodes, background anodes, and coincidence logic. Coincidence logic represents events such as adjacent anodes being hit, non-adjacent anodes being hit, TOF underflow and overflow, starts without stops, stops without starts, invalid events, and multiple starts before a stop. In Burst mode, the FSW preserves full 32 ESA 
Fig. 76 JADE valid mode transitions

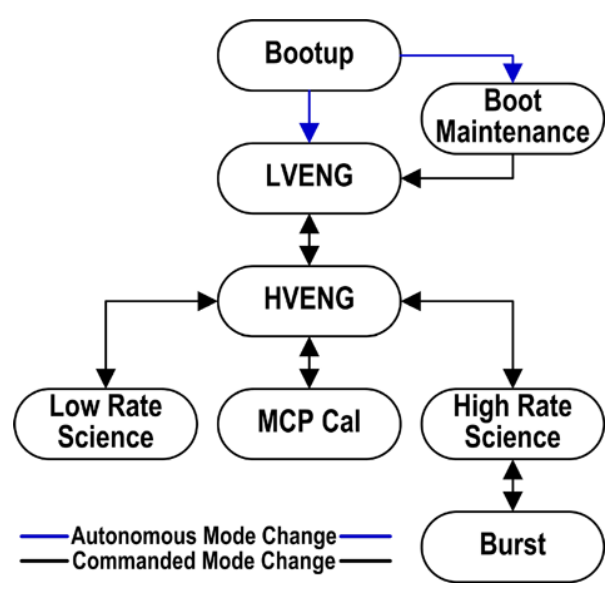

by 8 DFL resolution, but only keeps the counters for all starts, all stops, background, and invalid events. In High Rate mode, the FSW creates the same product as Burst, but with a reduced DFL resolution (summing adjacent DFL steps), for a data product of 32 ESA steps by 4 DFL steps by 4 counters. Additionally during HRS mode, the FSW produces a data product with the remaining 21 counters, but with no ESA/DFL resolution (all ESA and DFL steps summed together). During LRS and Cal modes, the FSW sums the histogram over a selectable number of seconds (nominally 5 or 10 minutes in LRS, and $30 \mathrm{~s}$ in Cal) then reduces the product to 25 individual counters, preserving no energy resolution (summing all ESA steps together).

\subsection{Software and Instrument Modes}

JADE has only two basic sets of modes: Boot Program and Science Program.

\subsubsection{Boot Program Mode}

Upon initial power on, JADE is in Bootup mode. The Boot Program starts execution of the Science Program from one of the two code images stored in the EEPROM (whichever has the first valid checksum). A number of checksums and memory tests are performed in Bootup mode. If all tests are passed, JADE autonomously transitions into the Low Voltage Engineering (LVENG) mode. Upon initial Bootup, JADE-I is powered on but all three JADE-Es remain off until commanded on.

If any of the conditions to enter the Science Program are not met, JADE enters Boot Maintenance mode rather than autonomously booting into LVENG. The following conditions result in entering Boot Maintenance mode: command sent and received, EDAC error, memory test error, bad checksum of both Science code images. JADE remains in Boot Maintenance until power cycled or explicitly commanded into the Science Program, where it enters LVENG.

\subsubsection{Science Program Modes}

JADE has 6 Science Program modes: LVENG, HVENG, MCP Calibration, LRS, HRS, Burst Science. Figure 76 shows the acceptable transition paths between the various modes. 
4.4.2.1 Low Voltage Engineering (LVENG) LVENG is entered immediately following successful Bootup and in this mode, the FSW first validates the Science Program Lookup Tables and then checks for the presence of the JSIB. If the JSIB is not present, or the Lookup Tables are corrupted, the Science Program stays in this mode and refuses to enter any other Science modes. In LVENG, all power supply voltages are set to 0 , all JADE-E sensors are powered off (JADE-I low voltage remains powered), stims are disabled, and data buffers are cleared. Once in LVENG, the FSW produces housekeeping data and waits for commanding to one of the other modes.

4.4.2.2 High Voltage Engineering (HVENG) Once commanded into HVENG, JADE-I is, and any number of the three JADE-E sensors can be, powered on. Default consists of all 3 JADE-E powered off, and macros enable the sensors for transition into the next mode. Upon initial entry into HVENG no voltages are changed. In each subsequent return to HVENG, the ESA and DFL are returned to the last values commanded while in HVENG. This ensures photoelectron rejection voltages are retained after the MCP voltages have been brought up after initial turn-on. Science and housekeeping data are produced in HVENG and JADE can only enter and leave this mode by command or autosafing.

4.4.2.3 MCP Calibration (Cal) In Cal mode, JADE-I is on and any number of the three JADE-E sensors can be on, with a default is all three JADE-Es powered on. All prior MCP and TOF voltages from HVENG are retained and MCP voltages are stepped via command or macro to generate a gain curve (counts vs MCP voltage).

4.4.2.4 Low Rate Science (LRS) Low rate science mode is used for the large portions ( $>90 \%$ ) of the Juno orbit away from Jupiter, where power needs to be conserved. In LRS, JADE-I and only one of the three JADE-E sensors is powered on. Deflector voltages are 0 for JADE-I and are swept via DFL sweep table for JADE-E. The inertial spin phase and inertial spin rate provided by spacecraft Attitude Control System (ACS) are used to map the JADE-I and JADE-E anodes into spin sectors (if no ACS message is received, the default spin phase is 0 and the default spin rate is set to the nominal spin rate of $2 \mathrm{rpm}$ ). These spin phase ordered observations are collapsed, saved and telemetered down at a low cadence, depending on the available telemetry (typically providing 5 or 10 minute temporal resolution for standard LRS but increasing temporal resolution to 30 or $60 \mathrm{~s}$ accumulation for intermediate rate LRS.

4.4.2.5 High Rate Science (HRS) High rate science is the primary science mode for JADE, and is the mode that JADE is in for each of the critical perijove intervals, which includes the critical auroral crossings. In HRS, JADE-I and all three JADE-E sensors are nominally on with the DFL and ESA voltages sweeping at high rate to attain high temporal resolution observations. The broadcast magnetic field vector onboard Juno is received by JADE and used to determine which of the three JADE-E sensors has positive deflection, which has negative deflection, and which remains undeflected. Deflector voltages are swept via table.

4.4.2.6 Burst Science (Burst) Burst science mode is simply an extension of HRS, but at higher resolution (no collapsing of dimensions and no accumulation of multiple samples). Data products are sent directly to the spacecraft over dedicated serial lines and include all ion species. Owing to the very large data content, only short intervals of Burst can be stored and fit into the JADE telemetry allocation. 


\subsubsection{Tables and Macros}

The bulk of JADE operations are controlled by internally stored tables and macros to minimize the need for ground commanding. These allow the sensors to operate under a series of operating regimes within an orbit based on a small number of stored commands loaded once per orbit.

\subsubsection{JADE Tables}

JADE Lookup Table (LUT) The JADE Lookup Table (LUT) is a partition in JADE memory that contains the definitions of tables, macros and parameters. The LUT contains default parameters, hidden parameters, ion species TOF definitions, voltage sweep tables, compression definition, MCP rate limiting definitions, macros, autosafing definitions, and the magnetometer parameters.

Upon transition from Bootup to LVENG, the Science Program performs a checksum and EDAC check of the table images stored in EEPROM. If table image 1 has no errors, it is used. If image 1 of a table has errors and image 2 does not, image 2 is used. If both table images have errors, JADE remains in LVENG mode. The error-free table is stored in SRAM, where it is used to define and execute Science Program tasks. The contents of the LUT can be updated in LVENG or HVENG modes. These updates can be temporary (written to SRAM; reverts to default upon power cycle) or permanent (written to EEPROM; establishes new default).

Parameter Tables All default parameters are captured within the LUT. Examples of these parameters are default sweep table for JADE-E and JADE-I, default JADE-E for LRS, and default additional ion species. These parameters can be updated individually using the Set Parameter command or as part of a LUT upload. Updates can be either temporary or permanent.

ESA \& DFL Sweep Tables When in specified science modes, the ESA and DFL voltages sweep according to predefined sweep tables stored in the LUT. In LRS, JADE-I has 4 ESA sweep tables available and JADE-E has 3. In LRS JADE-E also sweeps their DFL using one of their 3 available DFLs tables. In HRS and Burst, JADE-I has 2 sweep tables that define ESA + DFL voltages. JADE-E has 3 ESA sweep tables available, and the DFL voltages are set according to the Mag parameters table in order to track the magnetic field direction. Large tables like the ESA and DFL sweep tables are updated by either overwriting the whole sweep table or the entire LUT. Updates can be either temporary or permanent.

Ion TOF Tables The TOF of an ion is measured between the START and STOP signals generated by JADE-I electronics. For each of the 32 JADE-I energy steps, the JSIB converts the time between the START and STOP into digital numbers, and uses it for the subsequent ion data processing. The delta time between the START and STOP signals is proportional to the M/q of the ion and is used to distinguish ion species. See Sect. 3.3.2 for more detail. Large tables like the Ion TOF table can be updated by either replacing the whole sweep table or the entire LUT. Updates can be either temporary or permanent.

4.4.3.2 JADE Macros JADE macros are a series of relative-timed commands stored in the LUT. These macros are called using the Execute Macro commands. When JADE receives an Execute Macro command, it sends the series of time-tagged commands stored in SRAM. Note that these macros are completely internal to JADE and are different from spacecraft command blocks, although the concept behind spacecraft blocks and JADE macros is the same. 
There are 40 macro "slots" available for use in JADE; half contain at least one interactive command and half contain only non-interactive commands. A non-interactive command does not change power draw by any more than $1 \mathrm{~W}$. The first 20 macros are dedicated to interactive commands and contain commanding to change Science modes, decrease voltage prior to an orbital maneuver, increase voltages after an OTM, and prepare JADE to be turned off by the spacecraft. The second 20 macros contain only non-interactive commands that contain commanding to configure settings within a mode, i.e. change the LRS histogram accumulate rate, change the optional ion species transmitted. Large tables like the macros can be updated by either replacing the whole macro table or the entire LUT. Updates can be either temporary or permanent.

\subsubsection{Data Compression}

4.4.4.1 Lossy Compression Most JADE products are lossy compressed by JADE except for Ion Direct Events, HVENG products, and housekeeping telemetry. Burst products transmitted over the high speed interface are also not compressed by JADE although they are subsequently compressed by the spacecraft.

Two 32-8 bit compression tables are provided for LRS and Cal Science products. This compression is implemented as a binary search through a step table of 256 values, where at most 8 comparisons are made before determining the compressed value. In contrast, HRS utilizes two 16-8 bit LUT compression tables where the value to be compressed is treated as an index into the table. Before each data product gets lossy compressed to 8 bits, the Science Program subtracts the minimum data value from each product element and reports the subtracted value in telemetry.

The JADE compression cuts the data volume in half, while maintaining losses below $14 \%$ across the entire range of fluxes measured. Steps are spaced such that there is $\sim 4 \%$ error in 16-8 compression and up to $14 \%$ error in 32-8 compression. The data processing software assigns a value to the data that is the average value of the compression bin; for example, if data with original values of 1000-1040 were compressed together, the data processing software could output $1020 \pm 20$ as the value of the original data.

4.4.4.2 Lossless Rice Compression In addition to the lossy compression, most products are also subsequently lossless compressed using the Rice algorithm (exceptions are Ion Direct Events, HVENG products, and housekeeping). Burst products are not lossless compressed by JADE although they may subsequently be lossless compressed by the spacecraft. Lossless compression can be enabled/disabled via the Set Parameter command.

4.4.4.3 Internal Data Policing The JADE instrument can produce far more telemetry than the spacecraft can store for transmission to the ground. JADE uses lossless compression techniques to reduce data size, but the compression ratio varies. Once JADE reaches a predefined orbit data limit in the spacecraft, all remaining data in the orbit is lost. To avoid losing high-priority data, JADE monitors data volume and omits low priority data as needed.

The orbit is broken into time intervals with a data limit set for each interval. A priority mask, configurable as a table parameter, designates each data product as high or low priority. While data volume for an interval is below the limit, all products are produced. Once the limit has been reached, only the high-priority products are produced. This ensures highpriority products are transmitted regardless of how well they compress. If an interval ends up below the data limit, the excess data allocation is saved for future use. 


\subsubsection{Burst Data Flow}

There are two ways to record JADE Burst data: bin recording and continuous recording. JADE must be commanded into and out of these Burst modes. The time spent in Burst mode is called a record session.

4.4.5.1 Bin Recording Burst JADE records data as a series of bins, which are regions of memory designed to hold $N$ words of recorded burst data. The number of bins, size of bins, and amount of data recorded in the bin leading up to the quality factor data is configurable by command. During any one record session, all bins are the same size, with the number and size configurable via command, subject to a minimum of $10 \mathrm{~s}$ of data. Once the bin is no longer being written to, it is a "saved bin" and is typically associated with a Waves quality factor. If no Waves quality factors are received with empty bins remaining, JADE data fills them with high-rate data, and those bins are saved without an associated quality factor. When there are no remaining empty bins the spacecraft first records over any bins without an associated Waves quality factor in a circular manner. If there are no empty bins and no bins without an associated Waves quality factor, data with an associated higher Waves quality factor overwrites the lowest quality factor data.

Another key aspect of Burst is the "quality point," which is where the recorded data associated with the quality factor resides. As part of the configuration of bins (number of bins, size of bins, etc.), the number of 32-bit words desired before the quality point is specified. If, at the time the quality factor is received from Waves, the scratch bin contains the specified number of words or more, the recording is allowed to continue until exactly the number of specified words precedes the quality point. If there are less than the specified points, the scratch bin is allowed to fill, and the resulting quality point will not have the specified number of 32-bit words preceding the event. It is important to note that the timestamp associated with the quality factor is not where the quality point nominally resides.

4.4.5.2 Continuous Burst The second mechanism, continuous recording, is independent of the Waves instrument. This recording is used to turn on recording and record up to a commanded number of data words.

\subsubsection{MCP Rate Limiting}

MCPs may be damaged by very large count rates. To protect against this, a rate-limiting algorithm has been implemented (see Fig. 77). When a consecutive number of count rates (Persistence Count) exceed a specified threshold (Critical Maximum), the MCP supply voltage is reduced by a specified "Delta Voltage." As long as count rates exceed the threshold, the voltage continues to be reduced until count rates are at an acceptable level. Once acceptable, supply voltage remains reduced for a specified amount of time (Down Time). After the Down Time, the supply voltage is incremented by Delta Voltage to the original voltage. The controlling parameters for MCP rate limiting: Persistence Count, Critical Maximum, Delta Voltage, and Down Time are commendable, and held within the LUT. All parameters in the rate limiting algorithm are changeable/settable in an onboard parameter table.

\subsubsection{JADE Autosafing}

The vast majority of JADE operations occur while the spacecraft is not in contact. As such, it is necessary for JADE software to internally monitor housekeeping telemetry and enter a 


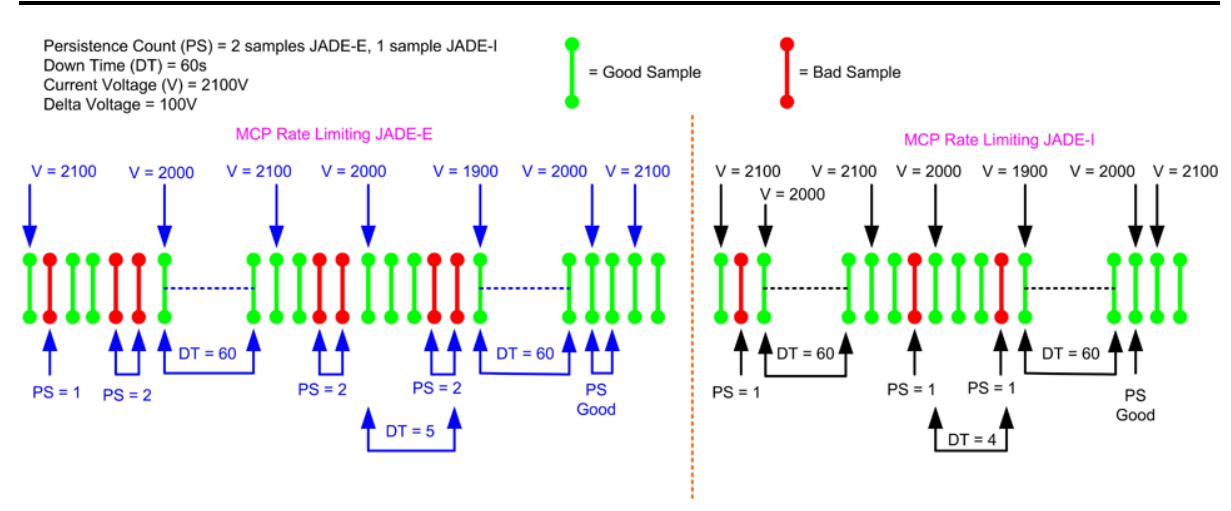

Fig. 77 MCP rate limiting algorithm

safe state if conditions suggest the possibility of hardware damage. Autosafing limits are set higher than the yellow and red limits monitored on the spacecraft in the real-time telemetry stream.

Within the LUT, all JSIB monitors have associated critical minimum and critical maximum values. JADE Autosafes if a telemetry point is consistently outside the region bracketed by these values over a specified persistence. Each monitored telemetry point also has an associated subsystem to be safed. For example if a telemetry point associated with safing JADE-E180 only is out of limits for the required persistence, only E180 is safed, and the rest of JADE continues taking data unaffected. Since JADE-I cannot be powered off without powering all of JADE off, when a JADE-I only telemetry point is tripped, it's high voltage is disabled.

\subsubsection{EDAC and Table Checksums}

Once the Science Program is running, JADE is very robust against bit errors. Error Detection and Correction (EDAC) scrubbing is performed continuously, which corrects single-bit errors. JADE is therefore not sensitive to single event upsets (SEUs). A double-bit error in SRAM causes a watchdog reset, and JADE reboots into LVENG. If a multiple-bit EDAC error in SRAM occurs, the Science Program writes the contents of the processor trace buffer into the EDAC Log before performing the watchdog reset.

\subsection{JADE Data Products}

JADE data files follow the Committee on Data Management and Computation (CODMAC) level descriptions, which are generic for use on science missions and not Juno-specific. The process follows level 1 (the raw data telemetered to Earth from Juno) to level 8 where userorientated information is provided, explaining why particular data is taken at a given time, and if there is any ancillary information associated with the dataset. Table 17 shows the CODMAC levels (different from NASA levels); not all levels are relevant to JADE, e.g. JADE does not provide any level 4 data products since there is no resampling of data.

For JADE, the data at different CODMAC levels is as:

- Level 1 Raw: All JADE instrument data delivered to Earth in Juno's telemetry stream, exactly as telemetered down. 
Table 17 CODMAC level descriptions

Level Description

1 Raw data: Telemetry with data embedded.

2 Edited data: Corrected for telemetry errors and split or decommutated into a data set for a given instrument. Sometimes called experimental Data Record. Data are also tagged with time and location of acquisition.

3 Calibrated data: Edited data that are still in units produced by instrument, but that have been corrected so that values are expressed in or are proportional to some physical unit such as radiance. No resampling, so edited data can be reconstructed.

4 Resampled data: Data that have been resampled in the time or space domains in such a way that the original edited data cannot be reconstructed. Could be calibrated in addition to being resampled.

5 Derived data: Derived results, as maps, reports, graphics, etc. NASA Levels 1C through 5.

6 Ancillary data: Non-science data needed to generate calibrated or resampled data sets. Consists of instrument gains, offsets; pointing information for scan platforms, etc.

7 Correlative data: Other science data needed to interpret spaceborne data sets. May include ground-based data observations such as soil type or ocean buoy measurements of wind drift.

8 User description: Description of why the data were required, any peculiarities associated with the data sets, and enough documentation to allow secondary user to extract information from the data.

$\mathrm{N}$ Not applicable.

- Level 2 Edited: Reformatted Engineering Data Records. Raw level 1 data is unpacked to separate science/engineering products and decompressed if required. Data are timeordered, redundant data packets are removed, and missing expected data flagged. No other calibration is carried out, e.g. data are left as counts per accumulation period for energy step $i$ and deflection step $j$.

- Level 3 Calibrated: Counts are converted to scientific units (i.e. counts per second, electron flux, energy is given in $\mathrm{eV}$ and deflection direction in degrees).

- Level 4 Resampled data: not relevant to JADE, data will not be resampled.

- Level 5 Derived: Pitch-angle distributions, electron/ion plasma parameters such as density, temperature and velocity. This may require additional calibrated information from other Juno instruments, i.e. magnetometer data for pitch angles.

- Level 6 Ancillary: Spacecraft position, velocity, orientation, spin-phase, quality flags, efficiency/voltage/energy tables used for level 3 products.

Figure 78 shows the planned data flow of the data from level 1 data from JPL (where the level 1 JADE data from Juno is gathered and transferred to the instrument team) through to level 3. The following subsections discuss currently planned data products at each level.

\subsubsection{Level 2 Reformatted Engineering Data Records}

4.5.1.1 Science Data Reformatted Engineering Data Records (REDRs) take the raw data and divide it into separate files depending on the JADE Data Product IDentifier (DPID) number. Table 18 separates these out by data type (rows) and telemetry mode (columns).

In addition to the above science data there are five operations-related data products concerning the whole JADE instrument suite: (1) BOOT_HK_Engineering/Housekeeping packet of the Boot Program (i.e. General monitors and power status'), (2) BOOT_MEM_ STAT-Memory Status packet of the Boot Program (i.e. checksums and counts of single/double-bit errors), MEM_DMP-Memory Dump packet (a complete dump of the 


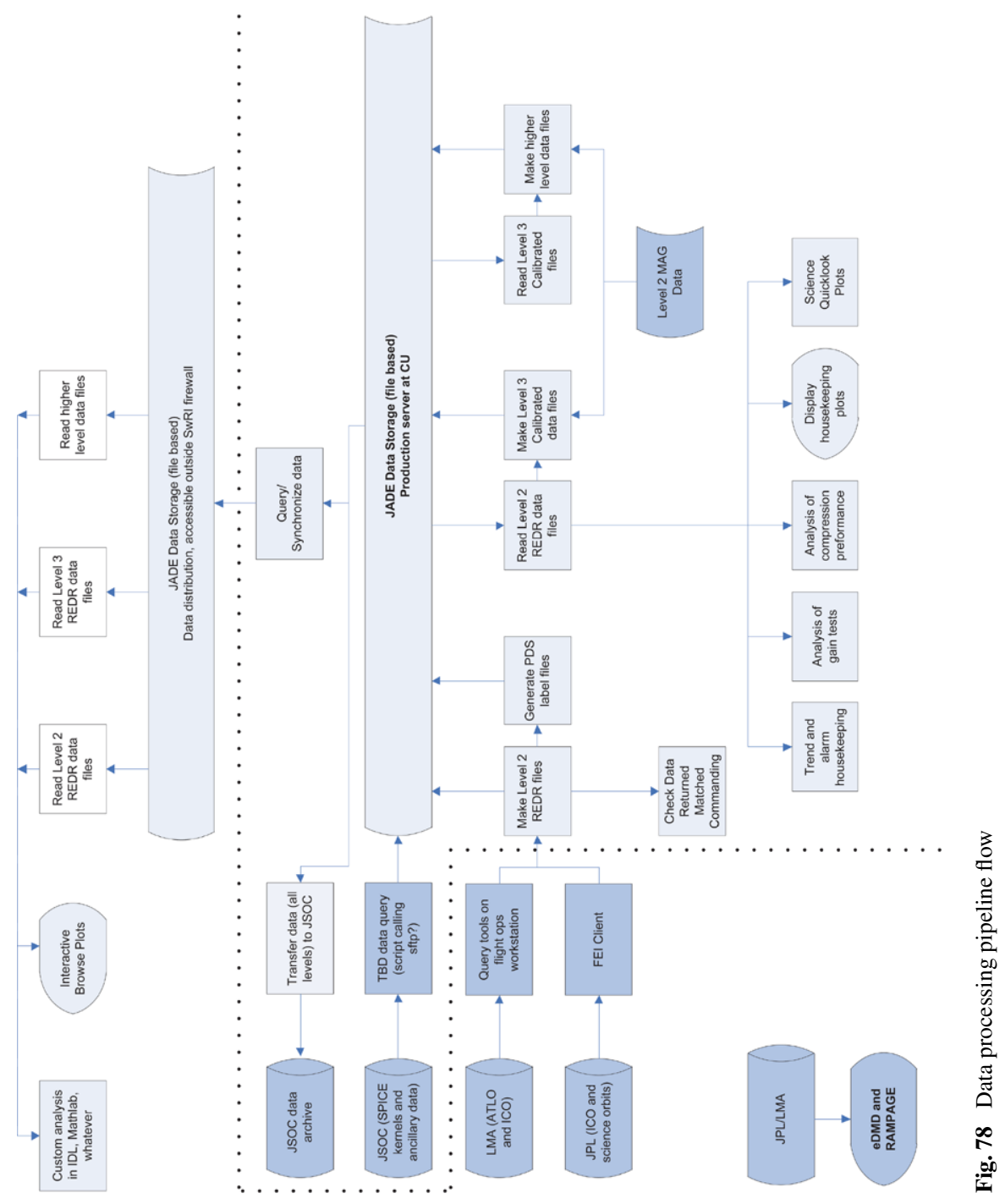


Table 18 All JADE science products

\begin{tabular}{llllll}
\hline $\begin{array}{l}\text { Type of } \\
\text { histogram }\end{array}$ & $\begin{array}{l}\text { Low rate } \\
\text { science }\end{array}$ & $\begin{array}{l}\text { High rate } \\
\text { science }\end{array}$ & $\begin{array}{l}\text { Burst } \\
\text { science }\end{array}$ & $\begin{array}{l}\text { MCP calibration } \\
\text { (ops use) }\end{array}$ & $\begin{array}{l}\text { High voltage } \\
\text { engineering science } \\
\text { (ops use) }\end{array}$ \\
\hline
\end{tabular}

\begin{tabular}{lccccc}
\hline Electron E060 & $\sqrt{ }$ & & & $\sqrt{ }$ & \\
Electron E180 & $\sqrt{ }$ & $\sqrt{ }$ & $\sqrt{ }$ & $\sqrt{ }$ & $\sqrt{ }$ \\
Electron E300 & $\sqrt{ }$ & & $\sqrt{ }$ & $\sqrt{ }$ & \\
Ion species 0 & $\sqrt{ }$ & $\sqrt{ }$ & $\sqrt{ }$ & $\sqrt{ }$ & \\
Ion species 1 & $\sqrt{ }$ & $\sqrt{ }$ & $\sqrt{ }$ & $\sqrt{ }$ \\
Ion species 2 & $\sqrt{ }$ & $\sqrt{ }$ & $\sqrt{ }$ & $\sqrt{ }$ \\
Ion species 3 & $\sqrt{ }$ & $\sqrt{ }$ & $\sqrt{ }$ & $\sqrt{ }$ & \\
Ion species 4 & $\sqrt{ }$ & $\sqrt{ }$ & $\sqrt{ }$ & $\sqrt{ }$ & \\
Ion species 5 & $\sqrt{ }$ & $\sqrt{ }$ & $\sqrt{ }$ & $\sqrt{ }$ & $\sqrt{ }$ \\
Ion species 6 & $\sqrt{ }$ & $\sqrt{ }$ & $\sqrt{ }$ & $\sqrt{ }$ & $\sqrt{ }$ \\
Ion species 7 & $\sqrt{ }$ & $\sqrt{ }$ & $\sqrt{ }$ & $\sqrt{ }$ & $\sqrt{ }$ \\
Ion time of flight & $\sqrt{ }$ & $\sqrt{ }$ & $\sqrt{ }$ & $\sqrt{ }$ \\
Ion logicals & $\sqrt{ }$ & $\sqrt{ }$ & $\sqrt{ }$ & $\sqrt{ }$ \\
Direct Events & $\sqrt{ }$ & $\sqrt{ }$ & $\mathrm{X}$ &
\end{tabular}

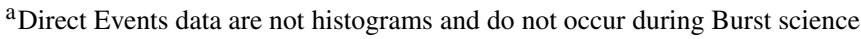

memory), SCI_ENG_-Engineering/Housekeeping packet of the Science Program (i.e. General monitors and power status), SCI_EVENT_Event Message packet of the Science Program (describes an unusual or unexpected occurrence, i.e. a command rejection).

4.5.1.2 Ion Species Identification The JADE-I sensor uses a loadable LUT to separate the measured events on the anodes to different species mappings. Events that register on a single or two adjacent STOP anodes get mapped to a species. Events that result in only a START event, Background events, or any events that get mapped to non-adjacent STOP anodes are not mapped to a species.

There are 8 potential species, numbered 0 through 7 . Species 0 is defined as any event that is seen on either a single or two adjacent STOP anodes. No START is required, so species 0 includes events that do not have a valid TOF measurement (a valid TOF measurement includes a valid START and STOP within a defined time range). For species 1-7 there is additional event masking possible so that events that have a 'bad' TOF can be masked out. Bad TOF events are durations shorter than $1 \mathrm{~ns}$ or longer than $330 \mathrm{~ns}$. Also, events that have multiple starts prior to the stop signal, or events that result in STOPS in adjacent anodes can be masked out of species 1-7. In the case of measuring STOPS in adjacent anodes, the event is mapped to the lower anode number in addition to incrementing a counter of the number of adjacent events. The mask setting is adjustable based on table values. At launch, adjacent events are included in species $1-7$, but bad TOF events and events with multiple starts are not.

Species 1 are all events that do have a valid TOF measurement. Species 1 is a subset of the species 0 events. Species 2-7 are reserved for specific mass ranges and each of species 2-7 are a subset of the species 1 data. Events are mapped to species 2-7 based on a LUT that defines a TOF range for each species and each ESA step. The TOF table in the LUT can be updated by uploading a new table or a new LUT. The species TOF table is currently defined 
to have species 2 for $M / q$ of 1 amu/q, species 3 for $M / q$ between 2 and 5, and species 4 to be for $M / q$ greater than $5 \mathrm{amu} / \mathrm{q}$.

4.5.1.3 Ion Direct Events There are two types of primary Science data products: histograms and direct events. Histograms are binned data and retain a complete history of the number of events that occurred within a given bin during an accumulation period. The duration of the accumulation period can be commanded, as noted earlier; low accumulation LRS has histograms that cover 300 or 600 s periods while intermediate accumulation LRS has histograms that cover 30 or 60 s periods. Because these events are simply counted, you can derive the number of particles from a given region from histograms but have no data on the order in which the particles were collected (i.e. if one section of the sky filled in preferentially earlier in the accumulation period and another section filled in later).

Direct events are individual events stored with time of collection, TOF, ESA and DFL information associated with them. A direct event carries with it all relevant information to describe the stored event, and thus is a much more detailed data product. Because the level of detailed information associated with just one direct event is large, telemetering many direct events quickly becomes a bandwidth issue. Juno is a telemetry-limited mission, so histograms are given priority storage. Direct events are stored on a low-priority basis and are transmitted when downlink allows.

4.5.1.4 Electron Data The electron data are returned as histograms of energy vs. pitch angle, as described in Sect. 4.3.4. For electrons, there is no complexity of TOF data, nor species separation, as there is for JADE-I.

\subsubsection{Level 3 Calibrated Data}

The measurements need to be converted to scientific units, which often requires knowing which plasma species is being measured. This is straightforward for electrons (negative ions are not expected at Jupiter). However, the ion detectors measure a variety of positively charged ions with different masses and different charges, i.e. $\mathrm{O}^{+}, \mathrm{S}^{++}, \mathrm{S}^{+}, \mathrm{O}^{++} \mathrm{S}^{+++}, \mathrm{H}^{+}$, in order of decreasing abundance. Therefore, the user must first partition the counts into their separate species, which is not possible if, as is usually the case, the velocity distributions overlap.

4.5.2.1 Conversion of Counts per Accumulation to Counts per Second The first level of calibration is to convert the counts per accumulation $\left(C_{\text {Accum. }}\right)$ into counts per second $\left(C_{s}\right)$. This is a simple division of:

$$
C_{s}=\frac{C_{\text {Accum }}}{d t}
$$

where $d t$ is the accumulation period, which is provided in each data file. Note this unit is independent of ion species or charge.

4.5.2.2 Convert to Differential Energy Flux Differential energy flux, DEF, is counts per second of a particular species (i.e. electrons or protons) divided by the geometric factor (see Sects. 3.2.1.3, 3.3.1.1 and Table 6) and has units of $\mathrm{cm}^{-2} \mathrm{sr}^{-1} \mathrm{~s}^{-1}$.

$$
D E F=\frac{C_{s}}{G}=\frac{C_{\text {Accum }}}{G d t}
$$

where the MCP efficiencies are already included in the effective geometric factors as they were derived from laboratory calibration measurements. 
4.5.2.3 Convert to Differential Number Flux Differential number flux, DNF, of a particular species is DEF divided by the energy ( $E$, in $\mathrm{eV})$ and modulus of charge ( $q$, in Coulombs) of the particle and has units of $\mathrm{cm}^{-2} \mathrm{sr}^{-1} \mathrm{~s}^{-1} \mathrm{~J}^{-1}$. For electrons or singularly charged ions, $q=1.602 \times 10^{-19} \mathrm{C}$.

$$
D N F=\frac{D E F}{E|q|}=\frac{C_{\text {Accum. }}}{E|q| G d t}
$$

4.5.2.4 Convert to Phase Space Density If the mass and charge of the species is known (or assumed), the Phase Space Density, PSD, is calculated from dividing the DEF by $v^{4}$ (where $v$ is found from $E / q=\frac{1}{2} m v^{2}$ ),

$$
P S D=\frac{D E F}{(2 E / m q)^{2}}=\frac{m^{2} q^{2} C_{\text {Accum }}}{4 E^{2} G d t}
$$

Note that this equation assumes there is zero spacecraft potential accelerating/decelerating the incident electrons/ions. This equation also shows the necessity of partitioning the ion counts into separate ion species. Note the strong dependence of this conversion of DEF to phase space density on the mass and charge of the particles: $\mathrm{H}^{+}$has an $(m q)^{2}=1 \mathrm{amu}^{2} \mathrm{e}^{2}$, $\mathrm{O}^{+}$has $(m q)^{2}=256\left(=16^{2}\right) \mathrm{amu}^{2} \mathrm{e}^{2}$, while $\mathrm{S}^{++}$has $(m q)^{2}=4096 \mathrm{amu}^{2} \mathrm{e}^{2}$. The net effect is that the resulting PSD, the desired scientific measurement, is very sensitive to assumption of what species is being detected.

\subsubsection{Level 5 Derived Data}

Once level 3 calibrated data exists; other data products may be derived. Examples include plasma density, flow velocity, isotropic temperatures, anisotropic temperatures and temperature anisotropies, and plasma $\beta$. Many methods for these derivations exist, however a few are discussed below.

4.5.3.1 Pitch-Angle Distributions In order to calculate pitch-angle distributions with respect to the magnetic field, one must know the magnetic field direction at the time of the particle measurement as accurately as possible, ideally with level 3 data from Juno's magnetometer. Calculating pitch-angle distributions requires reordering the data to refer to the particle's (ion or electron) direction of motion relative to the magnetic field direction, such that $0^{\circ}$ is parallel to the magnetic field, $90^{\circ}$ is perpendicular, and $180^{\circ}$ is anti-parallel. A dot product between the particle's flow direction and the magnetic field vector (in the same co-ordinate system) is then used to find the pitch angle. Note that the instrument FOV measures incoming particles, so that the flow FOV is the opposite direction (i.e. the instrument aperture must include the direction opposite to the particle motion).

4.5.3.2 Methods of Calculating Plasma Moments Methods of calculating single species moments of the velocity distribution function, $f(v)$, are well known (Baumjohann and Treumann 1999; Boyd and Sanderson 1969; Paschmann and Daly 1998) and particularly well suited to spinning spacecraft and electron data.

The $i$ th moment, $M_{i}$, of a given species is:

$$
M_{i}=\int f(v) v^{i} d^{3} v
$$


As such density, $n$, may be calculated by setting $i=0$ :

$$
n=M_{0}=\int f(v) d^{3} v
$$

$i=1$ provides $M_{1}$, which is equal to $n \boldsymbol{V}$ :

$$
n \boldsymbol{V}=M_{1}=\int f(v) v d^{3} v
$$

Since $n$ was found from the previous equation we can divide to obtain velocity, $\boldsymbol{V}$. Continuing to higher orders of $i$ can provide temperatures and pressures.

This technique is excellent for electron data where the plasma species has a known mass and charge (at Jupiter we do not expect to find the negative ions that are found near Titan or Enceladus at Saturn), and can give a cadence of one set of moments values per spin per sensor. Another benefit is that no plasma distribution (Maxwellian, Kappa, or other) is assumed.

However it is more complicated to use this technique for ion data because of the multiple species with differing masses and charges. Partitioning the ion data into single species to calculate $f(v)$ is complicated because the different species often overlap in velocity space. Therefore, a forward modeling method is generally preferred for magnetospheric ions.

\subsubsection{Methods of Calculating Plasma Parameters via Forward Modeling If multiple} plasma species exist and overlap in velocity space, then plasma parameters can also be obtained by a forward modeling. This requires assuming which plasma species are present and the nature of their velocity distribution (i.e. Maxwellian, kappa, etc.). These assumptions are then used to create a virtual model of the instrument response for a specified density, temperature and velocity of each plasma species. The simulated histogram (for a given density, temperature and velocity per plasma species) is then compared with the measured histogram from JADE, and the model plasma properties adjusted until the model matches the measured data (e.g. as quantified by a reduced Chi-square measure of goodness of fit).

This technique is computationally expensive, but does not require partitioning of the data in to separate species, allows exploration of the parameter space (usually not unique) that reasonably fit the data, and estimates statistical uncertainties of the output plasma properties. The disadvantage is it is necessary to assume the plasma species and distributions to use for the calculation; a poor assumption will give poor values.

4.5.3.4 Temperature Anisotropies and Plasma- $\beta$ Once densities and temperatures have been deduced by either moment summation or forward modeling, further values can be derived. For instance temperature anisotropy is the ratio of the perpendicular temperature to the parallel temperature. Likewise plasma $\beta$ is calculated as the ratio of plasma pressure to the magnetic pressure. Level 3 Juno magnetometer data provides $\boldsymbol{B}$, and combined with the level 5 JADE data of density, $n$, and temperature, $T$ to compute:

$$
\beta=\frac{n k_{B} T}{B^{2} / 2 \mu_{0}}
$$

where $k_{B}$ is the Boltzmann constant and $\mu_{0}$ is the permeability of free space. Further plasma properties such as Mach numbers, scale lengths, wave and collision frequencies can be calculated from the JADE ion and electron plasma parameters. 


\subsubsection{Level 6 Ancillary Data}

Level 6 data aid in understanding JADE data, but are not measured by JADE. For example, JADE data are time-stamped from the onboard Juno spacecraft clock and values included within all level 1 data records. For level 2 data, time is converted to UTC using NASA's Navigation and Ancillary Information Facility (NAIF) SPICE software suite (http://naif.jpl.nasa.gov). NAIF SPICE software is also used to generate all spacecraft orientation, position and velocity data. In addition, data from the magnetometer on Juno is used to convert JADE counting data in to pitch-angle distributions.

4.5.4.1 Time Time is expressed as a UTC string (yyyy-dddTHH:MM:SS.sss) and the corresponding Spacecraft clock time. Additionally, time to/since perijove is provided. These times and conversions are calculated using NAIF SPICE.

4.5.4.2 Spacecraft Position/Orientation Spacecraft position and velocity is provided in a variety of co-ordinate systems, including (but not limited to) Jupiter-centered radial distance, latitude and local time, SYS3 (with west longitude), RTN and J2000. The spacecraft's orientation is provided along with transformation matrices to convert this in to several other co-ordinate systems. Finally, the spacecraft spin rate and a spin phase are given, where zero spin phase is when Juno scans past ecliptic north. All this information originates from NAIF SPICE routines, so the particular SPICE kernels used are also noted.

4.5.4.3 JADE Instrument Information JADE can take a variety of data products, but often runs a subset in order to meet telemetry bandwidth allowances, therefore the data products "on" at any given time are listed. In addition, quality and warning flags are provided to help give context to the data. Magnetic field information is provided, along with a flag describing its source (i.e. onboard uncalibrated or ground-calibrated CODMAC level 3 data from the Juno MAG team).

Different energy tables can be used for each of the JADE instruments. This information includes which table is used, and efficiency parameters required to convert raw data in to calibrated units for CODMAC level 3 products.

\section{First Light Data}

\subsection{Overview}

Juno was launched on August 5, 2011 from Cape Canaveral Air Force Station. After commissioning of the spacecraft, instrument turn on began, with the initial JADE turn on and low voltage checkout on Oct 20, 2011 and high voltage checkout from November 14-18, 2011. By this time, the round trip light time (communications) was already over 5 minutes. While some final testing and tuning had to be deferred to a later date, all four JADE sensors were ramped up to their full high voltages. This included bringing all thirteen $10 \mathrm{kV}$ components to full operating voltage and ramping the MCP detectors up to their full operating voltages (saturation). Both JADE-I and all three JADE-Es immediately began taking science data in the solar wind, which demonstrated both their capabilities and scientific utility in the real space environment for the first time. This short section briefly highlights just a few of those "first light" observations.

Also after launch, the JADE-I sensor stabilized at a temperature $\sim 20{ }^{\circ} \mathrm{C}$ hotter than predicted. During the course of the investigation, we found that the JADE-I radiator was 
Fig. 79 JADE-E LRS data for a 10 min integration in the solar wind

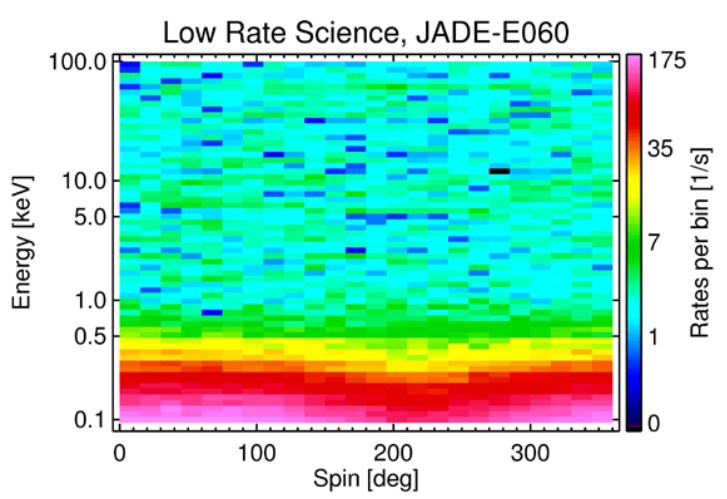

not located where the original thermal analysts had presumed it to be. Closeout photographs showed that JADE-I was built according to the drawings, but there was a disconnect between the drawings and the assumptions of the analysis. This thermal design issue could only possibly affect operation for the perihelion part of Juno's orbit only. At all larger heliocentric distances, including science operations at Jupiter, there is no impact to JADE whatsoever. To assess any possible perihelion issue, a new thermal model was created to match the as-built condition; this model predicted JADE-I perihelion temperatures a few ${ }^{\circ} \mathrm{C}$ warmer than protoflight thermal vacuum testing. To correlate the new thermal model, and to assess any risks of the perihelion thermal environment, we tested the flight-spare JADE-I sensor to well beyond that environment using the JPL solar illumination chamber. Detailed calibrations performed before and after testing showed that JADE-I should be totally unaffected by the perihelion environment.

\subsection{JADE-E}

We tested JADE in the LRS, HRS and Burst modes of operations during our initial turn on. The data presented here were collected during LRS in the solar wind. Figure 79 shows a spectrogram of energy versus spin angle taken by JADE-E060 (cf. Fig. 5) over a 10-min integration period, which corresponds to $\sim 10$ spacecraft spins. The energy ranges from $100 \mathrm{eV}$ to $100 \mathrm{keV}$ and the spin covers the entire $0^{\circ}-360^{\circ}$ rotation. The color codes the electron count rate per bin $\left(\sim 11.6 \%\right.$ in energy by $\left.15^{\circ}\right)$. Below $\sim 1 \mathrm{keV}$ the data are clearly ordered by spin phase and provide measurements of the solar wind electrons (core, halo and Strahl). Above $\sim 1 \mathrm{keV}$ the $2-3 \mathrm{~Hz}$ rates are caused by penetrating radiation (cosmic rays) and MCP internal noise; these low background rates indicate that JADE-E is ready to make the important Jovian electron measurements once it enters the Jovian magnetosphere.

Figure 80 shows energy spectra for all three JADE-E sensors where all the spin phases have been collapsed. During LRS only one JADE-E is powered, and thus the three JADE-E spectra were taken at different times (dates indicated in the plot). The penetrating background above $1 \mathrm{keV}$ has been averaged and subtracted from the spectra. We compare the JADE-E measurements to scaled (by distance and geometric factor) solar wind electron measurements (McComas et al. 1992) from the SWOOPS instrument on Ulysses. There is an excellent agreement between the spectra, which validates the JADE-E observations.

\subsection{JADE-I}

Like JADE-E, JADE-I was also operated in LRS, HRS and burst modes during HVCO, and was able to observe the solar wind. The energy spectra presented here (Fig. 81) were taken 
Fig. 80 Energy spectrum of solar wind electrons during a 10 min integration in LRS. The dashed line represents a typical solar wind electron spectrum scaled to JADE-E measurements from McComas et al. (1992)

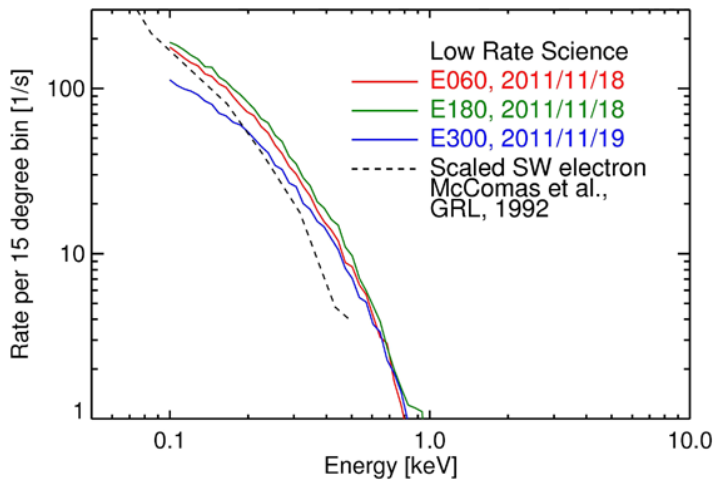

Fig. 81 Solar wind ion energy spectrum measured by JADE-I during an $\sim 2$ hour period while JADE-I was operated in HRS mode. The solid blue curve is for species 1 , which includes all mass per charges in the JADE-I measurement range. The dashed orange curve is for species 2 , which is for TOF values which map to $M / q$ of 1 (protons). Note how the distinctive second peak from alphas at $\sim 2 \mathrm{keV}$ is not seen in the species 1 data

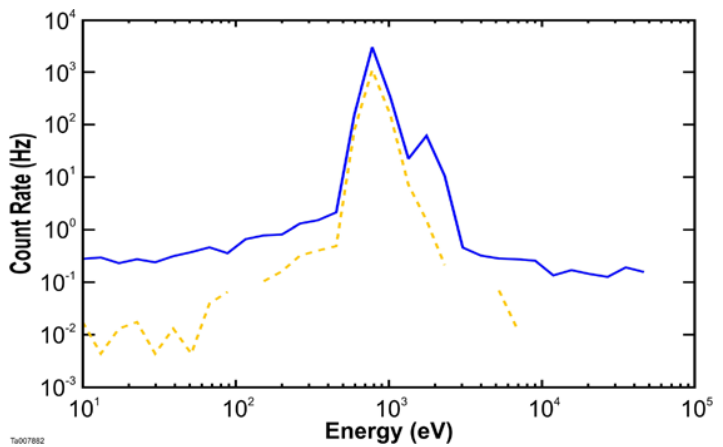

while in HRS mode. While operating in HRS mode, the ESA sweep table scans the full energy range of JADE-I (10 eV to $45 \mathrm{keV})$. Over the entire HVCO ( $\sim 6$ days), the solar wind was relatively stable, with a flow speed of $\sim 380-450 \mathrm{~km} \mathrm{~s}^{-1}$ as measured by ACE at 1 AU. Plotted in Fig. 81 is the energy spectra for species 1 in the solid blue line and species 2 in the dashed orange line. Species 2 was defined to be the TOF values that map to $M / q$ of 1 (protons). Species 1 is for any TOF value, and therefore includes alpha $(M / q=2)$ and heavier masses in this energy per charge range. The alphas are seen as a peak at $\sim 2 \mathrm{keV}$ in the species 1 data is successfully filtered out in the species 2 data.

Figure 82 shows energy per charge and TOF data while JADE-I was in LRS mode and using two different sweep tables. Overplotted on the data are curves that show contours of constant $M / q$ (of 1, 2, 4 and 8, solid lines) and constant incident velocity (dashed lines). For lower energies, the total energies of the particles inside the TOF section is dominated by the $10 \mathrm{kV}$ accelerating potential the particle experience as they are accelerated into the TOF section.

The top panel shows data taken when sweep with table 1 . This sweep table covers the same energy steps as were used during when in HRS mode and shown in Fig. 81. The bottom panel shows sweep table 2, which is reduced in range about the typical solar wind energies. The color bar on the right is common to both panels. The data had a background due to false coincidence events subtracted. The events at low TOF values $(<10)$ is due to electronic noise. The long tails to lower energies of each mass comes primarily from events measured by the anode that looks across the spacecraft deck face that is normal to the sun direction. This indicated that these lower energy tails come from some interaction with the solar wind with the spacecraft. While JADE-I was designed to measure magnetospheric plasmas and is 

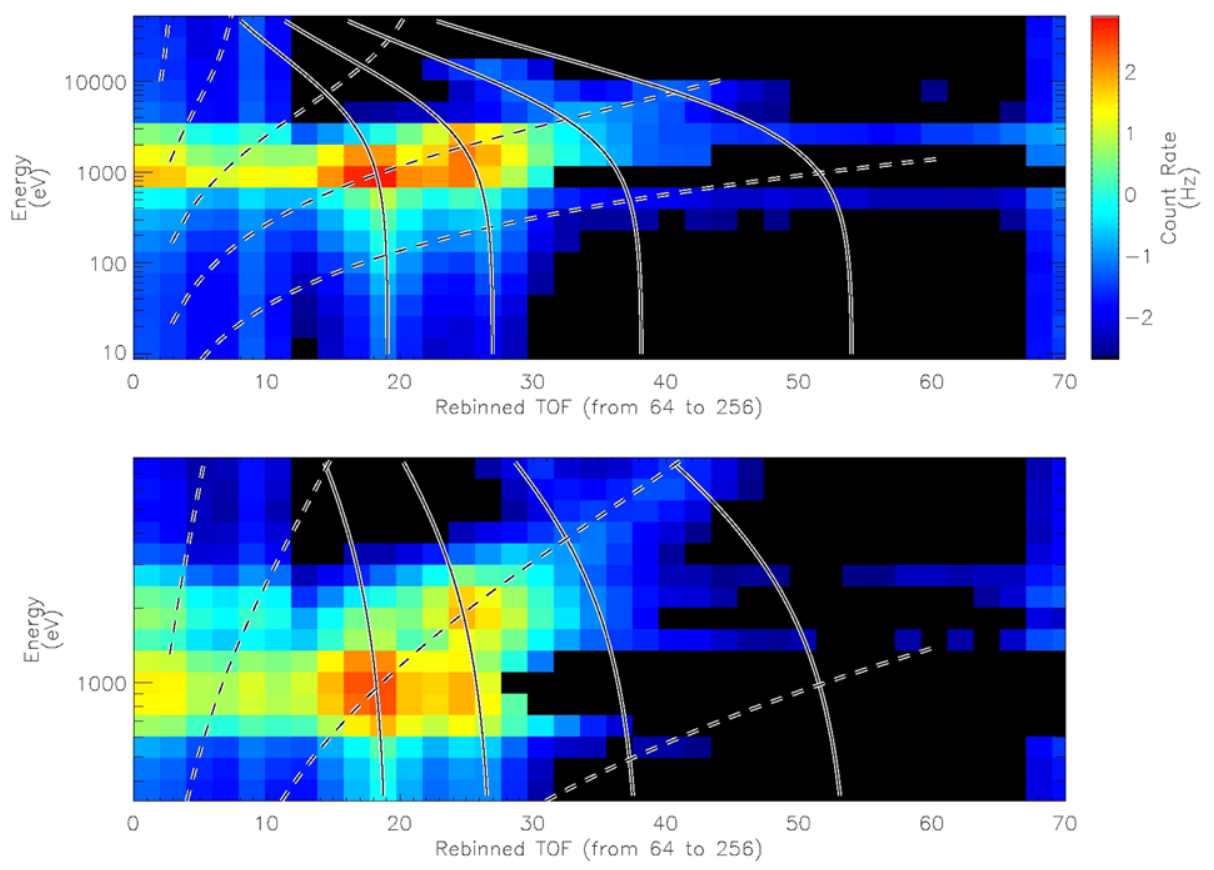

Fig. 82 Energy-TOF spectrograms for two different sweep tables. Overplotted on the data are curves of constant $M / q$ (solid lines) and constant incident velocity (dashed lines). The top panel shows data for sweep table 1, which covers from $10 \mathrm{eV}-40 \mathrm{keV}$ (the same energy steps as used during HRS mode and shown in Fig. 81). The bottom panel shows data for sweep table 2, which covers the typical solar wind energies of $0.3-8 \mathrm{keV}$

not capable of making high resolution measurements of the solar wind, it is clearly capable of measuring the protons, alpha, and heavier species in the solar wind.

\section{Summary and Conclusions}

The Jovian Auroral Distributions Experiment is a complete plasma instrument suite comprising three essentially identical electron sensors, a single ion sensor, and a common electronics box. Together, these provide unprecedented observations of the complicated Jovian magnetospheric electron and ion plasma populations and provide the core in situ measurements of the auroral particles populations needed to finally understand what creates the most intense auroral emissions in the solar system. Collectively, JADE provides a remarkably broad and detailed set of measurements of the Jovian auroral and magnetospheric plasmas, which will surely revolutionize our understanding of these important and complex regions.

While the EBox resides in the Juno Radiation Vault the JADE-I sensor and three JADE-Es look off of the edges of the spacecraft deck, with the latter being arrayed $120^{\circ}$ apart so as to be able to measure complete electron distributions at any spacecraft spin phase. The JADEEs measure electrons from $\sim 0.1$ to $100 \mathrm{keV}$ and provide detailed pitch-angle distributions at a $1 \mathrm{~s}$ cadence. JADE-I has an instantaneous field of view of $270^{\circ} \times 90^{\circ}$ and in just $4 \mathrm{~s}$, measures ions from $\sim 5 \mathrm{eV}$ to $\sim 50 \mathrm{keV}$ and separates the ion species from 1 to $50 \mathrm{amu}$ with $m / \Delta m \sim 2.5$; over each $30 \mathrm{~s}$ spacecraft rotation, JADE-I measures and analyzes ions from all directions in space. 


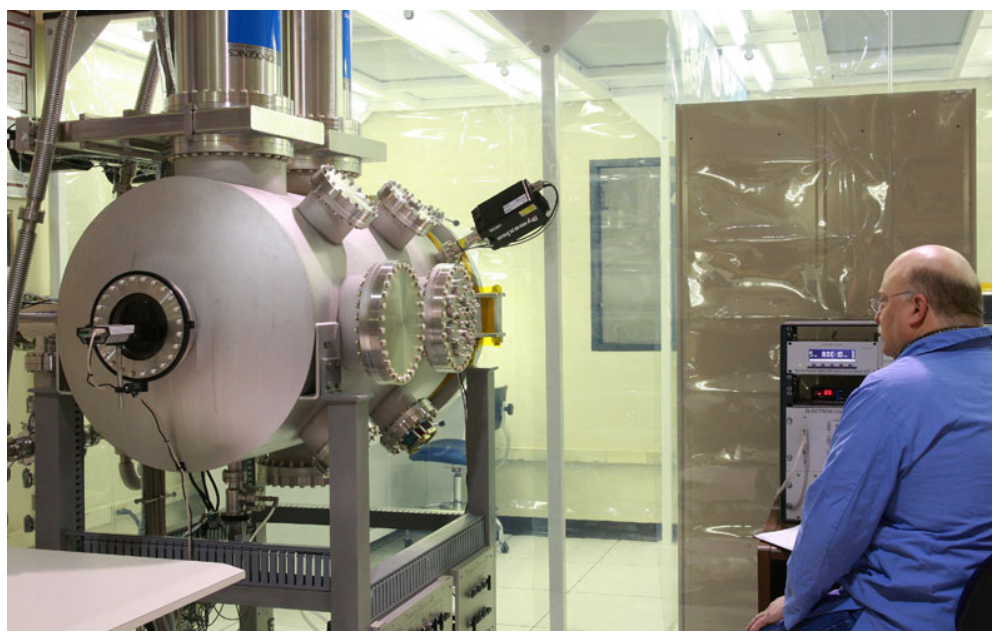

Fig. 83 The Southwest Research Institute Electron Calibration Facility. The main vacuum vessel has an approximate volume of 580 liters. Access to the interior of the chamber is through a Viton sealed door inside a class 10,000 clean tent, which includes a class 100 clean bench to facilitate final work on the instrument prior to installation into the vacuum chamber. Vacuum feed throughs of varying sizes circle the chamber and are accessible outside the clean tent

This paper describes in detail the JADE design, construction, calibration, planned science operations, data processing, and data products and documents the SwRI electron calibration facility, which was developed and used for the JADE-E calibrations. As such, this paper should be used as the citable reference for both JADE and for the SwRI electron calibration facility going forward.

Acknowledgements We are deeply indebted to all of the outstanding members of the JADE team who have made this exciting and challenging instrument suite a reality as well as to all of the dedicated people who have made the broader Juno program possible. This work was supported by NASA as a part of Juno mission, which is in NASA's New Frontiers Program.

Open Access This article is distributed under the terms of the Creative Commons Attribution License which permits any use, distribution, and reproduction in any medium, provided the original author(s) and the source are credited.

\section{Appendix: The Southwest Research Institute Electron Instrument Calibration Facility}

\section{A.1 Introduction}

The Southwest Research Institute Electron Calibration Facility (Fig. 83) is used to prototype, develop, and calibrate space flight plasma instruments. This facility was developed to support the calibration of the JADE-E sensors based on the design (and uses much of the existing infrastructure) of the Southwest Research Institute Calibration Facility described by McComas et al. (2009).

In Sect. A.2 we describe the vacuum system and general lab layout. Sect. A.3 describes the electron source and electron optics, including external magnet coils. The positioning system is described in Sect. A.4. 
Fig. 84 Schematic of the electron calibration facility vacuum components. Only oil-free vacuum pumps are used in this system to minimize the possibility of contamination to the instruments under test. Cleanliness of the vacuum is monitored with a $200 \mathrm{amu}$ Residual Gas Analyzer (RGA)

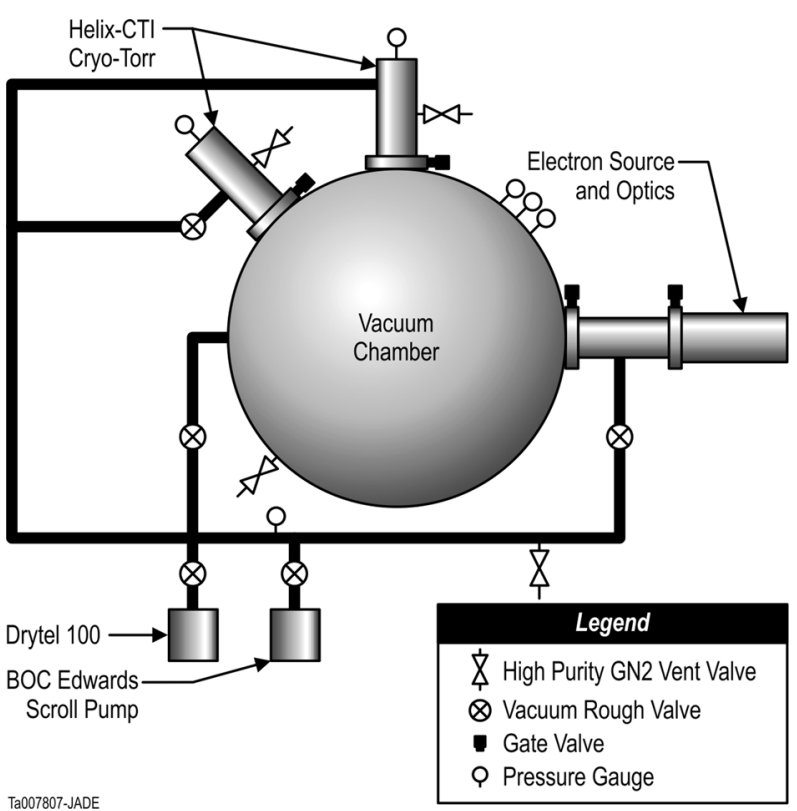

\section{A.2 Vacuum System and Lab Layout}

Space plasma instruments typically use either Microchannel Plates (MCPs) and/or Channel Electron Multipliers (CEMs) to detect single electrons, ions, or neutral atoms. Both of these types of detectors are extremely sensitive to contamination, especially complex hydrocarbons (e.g., McComas and Bame 1984; McComas et al. 1987, and references therein). Because it is not possible to replace detectors after launch, every effort must be made to maximize their useful lifetimes, which requires absolute cleanliness and the best possible vacuum conditions.

The control of the vacuum system was designed with instrument safety as the primary concern. All valves and pumps are controlled through a central control panel with internal logic that limits the actions available to the operator to prevent 'unsafe' actions. For example, venting of the system is not possible while the chamber is being actively pumped. If the facility loses power, all valves automatically close and the pumps turn off. The control system requires user intervention to bring the system back online to prevent a valve from opening into an unsafe state when power is re-established. The central control panel also isolates identified unsafe conditions or faults based on pressure and temperature monitors. If an overpressure is detected in one region of the vacuum system, valves close to isolate that portion of the system.

The vacuum chamber is divided into independently pumped subsections (Fig. 84). Each subsection can be valved off from the other portions and brought up to atmosphere while the others are held at high vacuum. These subsections are serviced by a common, oil-free, rough vacuum line that can pump them down independently or together from atmospheric pressure to a low pressure for cross-over to the cryogenic pumps.

The main vacuum vessel is a domed cylinder $76 \mathrm{~cm}$ (30 in) in diameter and $127 \mathrm{~cm}$ (50 in) long. The JADE-E sensors and any other test objects are mounted on a 3-axis positioning system (Sect. A.4). High vacuum pumping is supplied by 2 Helix-CTI Cryo-Torr 10 cryogenic pumps, each with a 3000 1/s pumping speed. These pumps were chosen for 
the pumping speed and cleanliness; if one pump suffers a failure and has to be isolated, the capacity of the other pump is sufficient to maintain the main vessel at HV levels, providing redundancy in the vacuum system. The vacuum pressure is monitored by two independent Pfeiffer PBR 260 Pirani/Hot Filament full range pressure gauges. The cleanliness of the vacuum is monitored with a Dycor Dymaxion Residual Gas Analyzer (RGA) that measures masses up to $200 \mathrm{amu}$. The ultimate pressure of the main vessel (empty) is $<1 \times 10^{-9}$ Torr, with the RGA typically showing essentially no mass contaminants ( $>50 \mathrm{amu}$ ) above $\sim 10^{-13}$ Torr.

The chamber door opens into and is accessed through a class 10,000 clean tent (Fig. 83), which contains a class 100 clean bench with ESD monitors. The ESD monitors are tied to the vacuum chamber walls and to Earth ground through grounding wells. High purity $\mathrm{N}_{2}$ is available in the clean tent for instrument purge. A series of 2.75, 8, 10, and 13.25 inch ConFlat flanges are available for electrical and mechanical feedthroughs. These feedthroughs are outside the clean tent to prevent compromising the clean area during instrument GSE set up. All flanges are the Con-Flat type except for the chamber doors, which use Viton seals.

The electron gun volume is connected to the main vessel through a $4.5^{\prime \prime}$ gate valve, which allows the electron gun to be serviced without bringing the main chamber up to atmospheric pressures. Once the electron gun volume is pumped to rough vacuum, high vacuum pumping is supplied by the 2 Helix-CTI Cryo-Torr 10 cryogenic pumps mounted on the main vessel.

All three vacuum subsections are serviced by a common rough vacuum line, which is pumped with a combination of a BOC Edwards scroll pump and a Drytel 100 series pump. This rough line is also used in the regeneration of the cryogenic pumps. Venting of all sections and pumps is performed with high purity (99.999\%) $\mathrm{N}_{2}$.

\section{A.3 Electron Gun and External Magnetic Coils}

The main electron source is a Kimball Physics model EMG-4212B with external power supply EGPS-4212B. The quoted energy range is 1 to $30 \mathrm{keV}$, but we are able to successfully operate the source down to $500 \mathrm{eV}$. Electrons are produced from a heated tantalum cathode, with a beam current up to $\sim 100 \mu \mathrm{A}$. The source is equipped with focusing optics and two sets of deflection plates. The quoted spot size ranges from $500 \mu \mathrm{m}$ to $25 \mathrm{~mm}$.

We also utilize an alternate Kimball Physics model FRA-2X1-2 lower energy, flood source: with energy range from $5 \mathrm{eV}$ to $1000 \mathrm{eV}$. The beam current for this source can be set up to $\sim 400 \mu \mathrm{A}$. The beam profile is Gaussian, but because there are no focusing optics for this source, the spot size depends on the working distance and energy. The source can be mounted on an UHV flange or separately on a bracket inside the chamber quite close to the experiment. Both the low and the high energy source are computer controlled.

The electron calibration facility is also equipped with three pairs of roughly square coils (not shown in Fig. 83) that cancel the Earth's magnetic field or create a stronger, nearly uniform magnetic field in a volume of about 1 square foot around the center of the chamber. The magnetic field is computer controlled and can be set up to $\sim 9$ Gauss in any direction with a maximum current of 15 A per coil.

\section{A.4 Positioning System}

The main vacuum chamber has a 3-axis positioning system that can handle instruments up to $\sim 20 \mathrm{~kg}$ and that fit within a volume of $25 \times 25 \times 25 \mathrm{~cm}$. These motions include a linear stage, which controls the horizontal position normal to the beam line and two nested, orthogonal rotational stages: horizontal (blue), outer rotation (yellow), and inner rotation 
Fig. 85 Cut away of main vacuum vessel showing the three stages of the positioning system: horizontal translation stage (blue), outer rotation stage (yellow), and inner rotation stage (green). For reference, a JADE-E sensor (white) is shown mounted onto the positioning system

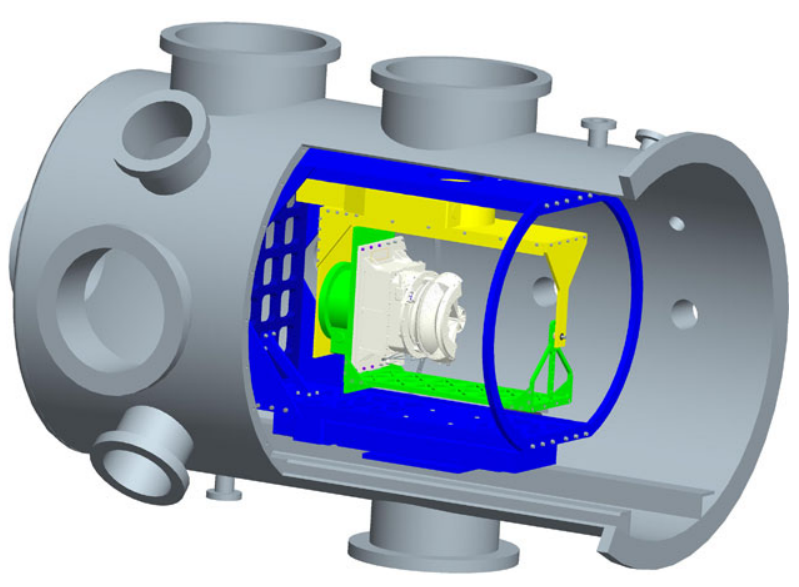

(green) in Fig. 85. These names were chosen to differ from standard Cartesian or polar coordinate names to prevent any confusion between the frame of reference of an instrument under test and that of the calibration facility.

The three stages are mounted so that both rotation stages ride on the horizontal stage, and the inner rotation stage rides on the outer stage. This means that for an instrument mounted with its aperture at the intersection of the two rotations axes, linear translations will not translate the aperture from the rotation axes. The rotation stages are mounted in an order that easily maps to a polar coordinate system with the outer stage mapping to the polar angle, and the inner stage mapping to the azimuth angle.

The inner stage is a Newport rotation stage model RV120 and the outer stage is a Newport rotation stage model RV160. Both rotation stages have a range of motion of $\pm 170^{\circ}$ with a resolution of $0.001^{\circ}$. The horizontal stage is a Newport linear stage model MTM250 with a range of motion of $\pm 12.5 \mathrm{~cm}$ with a resolution of $1 \mu \mathrm{m}$. All stages are controlled by a Newport XPS motion controller, which is commanded either through a hand held controller accessible within the clean tent, or through computer control that can carry out intricate motion scripts.

\section{References}

A. Adriani et al., The Jovian Infrared Auroral Mapper (JIRAM). Space Sci. Rev. (2013, this issue)

F. Bagenal, P.A. Delamere, Mass and energy flow through the magnetospheres of Jupiter and Saturn. J. Geophys. Res. 116, A05209 (2011)

F. Bagenal et al., Magnetospheric science objectives of the Juno mission. Space Sci. Rev. (2013, this issue)

S.J. Bame, D.J. McComas, B.L. Barraclough, J.L. Phillips, K.J. Sofaly, J.C. Chavez, B.E. Goldstein, R.K. Sakurai, The Ulysses solar wind plasma experiment. Astron. Astrophys. Suppl. Ser. 92, 237-265 (1992)

W. Baumjohann, R.A. Treumann, Basic Plasma Physics (Imperial College Press, London, 1999)

S. Bolton et al., The Juno mission, Space Sci. Rev. (2013, this issue)

T.J.M. Boyd, J.J. Sanderson, Plasma Dynamics (Thomas Nelson and Sons, London, 1969)

C.W. Carlson, R.F. Pfaff, J.G. Watzin, The Fast Auroral SnapshoT (FAST) mission. Geophys. Res. Lett. 25(12), 2013-2016 (1998)

J.T. Clarke, J. Ajello, G. Ballester, L. Ben Jaffel, J. Connerney, J.-C. Gerard, G.R. Gladstone, D. Grodent, W. Pryor, J. Trauger, J.H. Waite, Ultraviolet emissions from the magnetic footprints of Io, Ganymede and Europa on Jupiter. Nature 415, 997 (2002)

J. Clarke, D. Grodent, S. Cowley, E. Bunce, P. Zarka, J. Connerney, T. Satoh, Jupiter's aurora, in Jupiter: Planet, Satellites, Magnetosphere, ed. by F. Bagenal, T.E. Dowling, W.B. McKinnon (Cambridge University Press, Cambridge, 2004) 
J.E.P. Connerney, M.H. Acuna, N.F. Ness, Modeling the Jovian current sheet and inner magnetosphere. J. Geophys. Res. 86, 8370-8384 (1981)

J.E.P. Connerney, M.H. Acuna, N.F. Ness, T. Satoh, New models of Jupiter's magnetic field constrained by the 10 flux tube footprint. J. Geophys. Res. 103, 11929-11939 (1998)

J.E.C. Connerney et al., MAG. Space Sci. Rev. (2013, this issue)

D.A. Dahl, SIMION for the personal computer in reflection. Int. J. Mass Spectrom. 200, 3-25 (2000)

P.A. Delamere, F. Bagenal, Solar wind interaction with Jupiter's magnetosphere. J. Geophys. Res. 115, A10201 (2010)

N. Divine, H.B. Garrett, Charged particle distribution in Jupiter's magnetosphere. J. Geophys. Res. 88(A9), 6689-66903 (1983)

H.O. Funsten, D.J. McComas, E.E. Scime, Low-energy neutral-atom imaging techniques, in SPIE Proceddings, vol. 2008 (1993), pp. 93-104

H.O. Funsten, R.W. Harper, D.J. McComas, Absolute detection efficiency of space-based ion mass spectrometers and neutral atom imagers. Rev. Sci. Instrum. 76(5), 053301 (2005)

R. Gladstone et al., UVS. Space Sci. Rev. (2013, this issue)

J.T. Gosling, The solar wind, in Encyclopedia of the Solar System, 2nd edn., ed. by L.-A. McFadden, P.R. Weissman, T.V. Johnson (Academic Press, San Diego, 2007), pp. 99-116

C.H. Hansen et al., Junocam Space Sci. Rev. (2013, this issue)

S.P. Joy, M.G. Kivelson, R.J. Walker, K.K. Khurana, C.T. Russell, T. Ogino, Probabilistic models of the Jovian magnetopause and bow shock locations. J. Geophys. Res. 107(A10), SMP 17-1 (2002). doi:10.1029/2001JA009146

O. Keski-Rahkonen, M.O. Krause, Relativistic effects in the calibration of electrostatic electron analyzers. I. Toroidal analyzers. J. Electron Spectrosc. Relat. Phenom. 13(2), 107-112 (1978)

K.K. Khurana, M.G. Kivelson, V.M. Vasyliunas, N. Krupp, J. Woch, A. Lagg, B.H. Mauk, W.S. Kurth, The configuration of Jupiter's magnetosphere, in Jupiter: Planet, Satellites, Magnetosphere, ed. by F. Bagenal, T.E. Dowling, W.B. McKinnon (Cambridge University Press, Cambridge, 2004)

N. Krupp, V.M. Vasyliunas, J. Woch, A. Lagg, K.K. Khurana, M.G. Kivelson, B.H. Mauk, E.C. Roelof, D.J. Williams, S.M. Krimigis, W.S. Kurth, L.A. Frank, W.R. Paterson, Dynamics of the Jovian magnetosphere, in Jupiter: Planet, Satellites, Magnetosphere, ed. by F. Bagenal, T.E. Dowling, W.B. McKinnon (Cambridge University Press, Cambridge, 2004)

W.S. Kurth et al., Waves Space Sci. Rev. (2013, this issue)

B. Mauk et al., JEDI Space Sci. Rev. (2013, this issue)

W. McAlpine, Juno 2011LD-19oct2016AD charged-particle radiation flux, JPL Document No. 5132-07-017, February 14 (2007)

D.J. McComas, S.J. Bame, Channel multiplier compatible materials and lifetime tests. Rev. Sci. Instrum. 55(4), 463-467 (1984)

D.J. McComas, F. Bagenal, Jupiter: a fundamentally different magnetospheric interaction with the solar wind. Geophys. Res. Lett. 34, L20106 (2007)

D.J. McComas, J.R. Baldonado, S.J. Bame, B.L. Barraclough, Channel electron multiplier compatibility with viton and Apiezon-L vacuum grease. Rev. Sci. Instrum. 58, 2331-2332 (1987)

D.J. McComas, S.J. Bame, W.C. Feldman, J.T. Gosling, J.L. Phillips, Solar wind halo electrons from 1-4 AU. Geophys. Res. Lett. 19(12), 1291-1294 (1992)

D.J. McComas, F. Allegrini, C.J. Pollock, H.O. Funsten, S. Ritzau, G. Gloeckler, Ultra-thin ( $\sim 10 \mathrm{~nm})$ carbon foils in space instrumentation. Rev. Sci. Instrum. 75(11), 4863-4870 (2004)

D.J. McComas, F. Allegrini, F. Bagenal, F. Crary, R.W. Ebert, H. Elliott, A. Stern, P. Valek, Diverse plasma populations and structures in Jupiter's magnetotail. Science 318, 217-220 (2007)

D.J. McComas et al., The Two Wide-angle Imaging Neutral-atom Spectrometers (TWINS) NASA missionof-opportunity. Space Sci. Rev. 142, 157-231 (2009). doi:10.1007/s11214-008-9467-4

R.L. McNutt Jr., D.K. Haggerty, M.E. Hill, S.M. Krimigis, S. Livi, G.C. Ho, R.S. Gurnee, B.H. Mauk, D.G. Mitchell, E.C. Roelof, D.J. McComas, F. Bagenal, H.A. Elliott, L.E. Brown, M. Kusterer, J. Vandegriff, S.A. Stern, H.A. Weaver, J.R. Spencer, J.M. Moore, Energetic particles in the Jovian magnetotail. Science 318, 220-222 (2007)

National Research Council, New Frontiers in the Solar System: An Integrated Exploration Strategy (National Academies Press, Washington, 2003)

T.G. Northrop, The adiabatic motion of charged particles, in Inter-science Tracts on Physics and Astronomy, (1963)

G. Paschmann, P.W. Daly, Analysis Methods for Multi-Spacecraft Data. ISSI Scientific Reports Series SR001, ESA/ISSI, Vol. 1. ISBN 1608-280X, 1998

G. Paschmann, S. Haaland, R. Treumann (eds.), Auroral Plasma Physics (Kluwer Academic, Dordrecht, 2003) 
B.M. Randol, H.A. Elliott, J.T. Gosling, D.J. McComas, N.A. Schwadron, Observations of isotropic interstellar pick-up ions at 11 and 17 AU from New Horizons. Astrophys. J. 755, 75 (2012)

J.G. Roederer, Dynamics of geomagnetically trapped radiation, in Physics and Chemistry in Space, vol. 2 (Springer, Berlin, 1970)

D. Santos-Costa, Modeling modélisation des ceintures de radiation d'électrons de Jupiter internes à Io, ENSAE PhD report (2001)

D. Santos-Costa, S.J. Bolton, Discussing the processes constraining the Jovian synchrotron radio emission's features. Planet. Space Sci. 56(3-4), 326-345 (2008)

D. Santos-Costa, S.A. Bourdarie, Modeling the inner Jovian electron radiation belt including non-equatorial particles. Planet. Space Sci. 49, 303-312 (2001)

M. Schulz, M.J. Lanzerotti, Particle diffusion in the radiation belts, in Physics and Chemistry in Space, vol. 7 (Springer, Berlin, 1974)

Y.-J. Su, R.E. Ergun, F. Bagenal, P.A. Delamere, Io-related Jovian auroral arcs: modeling parallel electric fields. J. Geophys. Res. 108, 15 (2003)

N. Thomas, F. Bagenal, T.W. Hill, J.K. Wilson, The Io neutral clouds and plasma torus, in Jupiter: Planet, Satellites, Magnetosphere, ed. by F. Bagenal, T.E. Dowling, W.B. McKinnon (Cambridge University Press, Cambridge, 2004)

D.J. Williams, R.M. Thorne, B. Mauk, Energetic electron beams and trapped electrons at Io. J. Geophys. Res. 104, 14739 (1999)

T. Zurbuchen, P. Bochsler, F. Scholze, Reflection of ultraviolet light at $121.6 \mathrm{~nm}$ from rough surfaces. Opt. Eng. 34(5), 1303 (1995) 